\title{
Lead-Free Perovskite Materials for Solar Cells
}

Check for

updates

\section{Cite as}

Nano-Micro Lett.

(2021) 13:62

Received: 19 August 2020

Accepted: 4 December 2020

Published online: 25 January 2021

(C) The Author(s) 2021

\author{
Minghao Wang ${ }^{1}$, Wei Wang ${ }^{1}$, Ben $\mathrm{Ma}^{1}$, Wei Shen ${ }^{1}$, Lihui Liu ${ }^{1}$, Kun Cao ${ }^{1}$, \\ Shufen Chen ${ }^{1,2}$, Wei Huang ${ }^{1,2} \bowtie$
}

\section{HIGHLIGHTS}

- The toxicity issue of lead-based halide perovskites hinders theirs large-scale commercial applications in solar cells.

- A variety of non- or low-toxic perovskite materials have been used for development of environmentally friendly lead-free perovskite solar cells, some of which show excellent optoelectronic properties and device performances.

- At present, more new lead-free perovskite materials with tunable optical and electrical properties are urgently required to design highly efficient and stable lead-free perovskite solar cells.

ABSTRACT The toxicity issue of lead hinders large-scale commercial production and photovoltaic field application of lead halide perovskites. Some novel non- or low-toxic perovskite materials have been explored for development of environmentally friendly lead-free perovskite solar cells (PSCs). This review studies the substitution of equivalent/ heterovalent metals for $\mathrm{Pb}$ based on first-principles calculation, summarizes the theoretical basis of lead-free perovskites, and screens out some promising lead-free candidates with suitable bandgap, optical, and electrical properties. Then, it reports notable achievements for the experimental studies of lead-free perovskites to date, including the crystal structure and material bandgap for all of lead-free materials and photovoltaic performance and stability for corresponding devices. The review finally discusses challenges facing the successful development and commercialization of lead-free PSCs and predicts the prospect of lead-free PSCs in the future.

KEYWORDS Solar cells; Perovskite; Lead-free; First-principles calculation; Photovoltaic

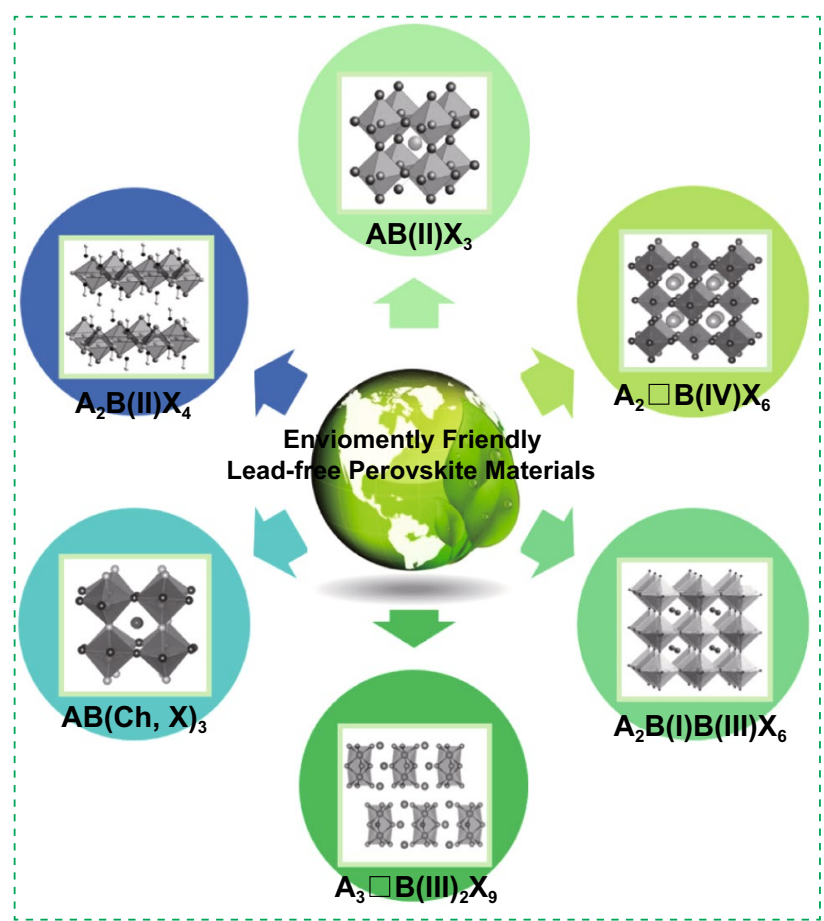

Shufen Chen, iamsfchen@njupt.edu.cn; Wei Huang, wei-huang@njtech.edu.cn; iamdirector@fudan.edu.cn

1 Key Laboratory for Organic Electronics and Information Displays \& Jiangsu Key Laboratory for Biosensors, Institute of Advanced Materials (IAM), Jiangsu National Synergetic Innovation Center for Advanced Materials (SICAM), Nanjing University of Posts \& Telecommunications (NUPT), 9 Wenyuan Road, Nanjing 210023, People's Republic of China

2 Frontiers Science Center for Flexible Electronics, Xi' an Institute of Flexible Electronics (IFE) and Xi' an Institute of Biomedical Materials \& Engineering, Northwestern Polytechnical University, 127 West Youyi Road, Xi'an 710072, People's Republic of China 


\section{Introduction}

As a major driving force of the world today, traditional fossil fuels have been essential from the first industrial revolution. Although previous industrial revolutions have brought human society into an unprecedented boom era, they have simultaneously caused tremendous energy and resource consumption and aggravated the contradiction between human and nature, making us have to pay a huge environmental price and ecological cost. With the fast development of world's economy and social productive forces, the requirement of energy has also been growing rapidly and the traditional fossil energy has been incapable of meeting our demands. So entering the twenty-first century, humanity has been facing unprecedented challenges of global crises of energy and resource, environment, ecology, and climate change. All of these issues have initiated the fourth industrial revolution, namely the green industrial revolution. At this time, solar energy as the initial source of all energy inevitably becomes new support for human and social development due to its advantages of pollution-free, renewability, huge energy, and so on. Optoelectronic conversion as one of the major utilizations of solar energy has been widely researched in the past several decades, and solar cells belong to the most important type of optoelectronic converters. The development of low-cost, high-efficiency solar cells has become a central issue in recent years. And perovskite solar cells (PSCs), as a promising class of solar cells family, have attracted intensive attention in the past decade due to high absorption coefficient, excellent bipolar charge mobility, long carrier diffusion length, low exciton binding energy, low trap state density, and tunable bandgap.

The power conversion efficiency (PCE) of PSCs based on lead $(\mathrm{Pb})$ perovskites has been dramatically improved from the initial $3.8 \%$ to recently certified value of $25.2 \%$ [1-24]. However, there exist unavoidable shortcomings in these Pb-based high-efficiency PSCs (e.g., $\mathrm{MAPbI}_{3}, \mathrm{FAPbI}_{3}$, $\left.\mathrm{Cs}_{0.05} \mathrm{FA}_{0.85} \mathrm{MA}_{0.10} \mathrm{~Pb}\left(\mathrm{I}_{0.97} \mathrm{Br}_{0.03}\right)_{3}\right)$, that is, the element lead is toxic to the environment and organisms and difficult to discharge from the body. Research indicates that the contamination of lead ions to soil and water sources is permanent and generates a very serious negative impact on human, animal, and plant survival [25-34]. The human's nervous, digestive and blood systems eventually show functional disorders in the case of lead poisoning since lead can enter human's body, bind with enzyme and finally store in soft tissues and bones such as spleen, kidney, liver, and brain through blood circulation. Generally, most people show lead toxic symptoms when lead intake reaches about $0.5 \mathrm{mg} / \mathrm{day}$ [25-27]. Therefore, to guarantee human's safe and pollutionfree natural environment it is necessary to develop some non- or low-toxic metal ions to replace lead as perovskite absorbers of PSCs [28-34].

Generally, metal halide perovskites have a universal chemical formula of $\mathrm{ABX}_{3}$, where $\mathrm{A}$ is an organic cation, $B$ is a metal cation and $X$ is a halogen anion [35-55]. Here, the organic cation usually includes methylammonium (MA), formamidinium (FA), Cs, or their mixture, and the halogen anion consists of $\mathrm{Cl}, \mathrm{Br}$, I or their mixture. For $\mathrm{B}$, previous work has showed that less toxic ions like $\mathrm{Sn}^{2+}, \mathrm{Bi}^{3+}, \mathrm{Ge}^{2+}$, $\mathrm{Sb}^{3+}, \mathrm{Mn}^{2+}$, and $\mathrm{Cu}^{2+}$ could be used as an alternative ion to $\mathrm{Pb}^{2+}$ in perovskites to constitute a new lead-free perovskite structure [56-63]. The introduction of these metal cations not only increases the diversity of perovskite species, but also enhances environmentally friendly features of PSCs.

This review mainly focuses on various lead-free perovskite materials and related solar cell applications by summarizing recent work about theoretical bases and experimental studies of those lead-free perovskites. Specifically, it summarizes the replacement of $\mathrm{Pb}$ with other possible isovalent and heterovalent elements proposed recently. Herein, the isovalent elements consisting of Sn (II), Ge (II) and divalent transition metals (e.g., $\mathrm{Cu}, \mathrm{Fe}, \mathrm{Zn}$ ), and the heterovalent elements containing Bi(III), Sb (III), Sn (IV), Ti (IV) and double cations of $\operatorname{Ag}(\mathrm{I}) \mathrm{Bi}(\mathrm{III})$ are summarized. The crystal structure and bandgap for all of lead-free materials and their corresponding photovoltaic performance and stability are included. And by comparing various types of lead-free perovskite materials, we finally predict development prospects of lead-free perovskite materials in the future.

\section{Theoretical Bases of Lead-Free Hybrid Perovskites}

First-principles calculation based on density functional theory (DFT) is a modeling method for simulating the electronic structures of many-body systems from atomicscale quantum mechanics. This method has been used as an important tool for the study of lead-free perovskites [64, 65]. Electronic structures of various lead-free perovskites 
were calculated by researchers to simulate their electron/ hole effective masses, theoretical absorption spectra, carrier mobilities, bandgaps, and other properties related to their potential solar cell applications by first-principles calculations in order to find the optimal $\mathrm{MAPbI}_{3}$ substitute [67-70]. Generally speaking, there exist a variety of options of exchange-correlation functions in DFT calculations, which will qualitatively affect the result of DFT calculations. In the past research work, researchers often chose local density approximation (LDA) and generalized gradient approximation (GGA) functionals. Although their functions are relatively simple, the calculation results are generally more reliable. But this does not mean that there are no problems. LDA and GGA exhibit serendipitous cancellations between their exchange-correlation parts, which lead to unphysical electron self-interactions. This result further causes the local (LDA) and semilocal (GGA) functions to essentially underestimate the bandgaps of solids by $30-100 \%$ [67, 68]. To solve such problems, methods/functions including the many-body perturbation (GW) approximation [65], timedependent DFT (TDDFT) [66], PBE0, HSE03, and HSE06 hybrid functionals [64-70], and more recent delta selfconsistent-field method have been developed and applied to recent theoretical calculations of lead-free perovskites. It is worth emphasizing that in the presence of heavy atoms, spin-orbit coupling (SOC) must be included in the calculation to achieve a more accurate prediction of the electronic structure.

So far, researchers have already had a certain understanding of the lead-free perovskite system after several years of targeted research. The core of research on lead-free perovskite materials is the replacement of $\mathrm{Pb}$ element. Researchers have screened out a series of elements that could replace $\mathrm{Pb}$ through theoretical calculations (Fig. 1).

Filip et al. considered stability and desired bandgap as two concurrent prerequisites for suitable candidates of lead-free perovskites [66]. They used randomly displaced structures (atom positions and lattice parameters) to investigate the stability of possible lead-free perovskites. Making the stability and desired calculated bandgap as two concurrent conditions, the crystal structure retains the perovskite geometry after relaxation of the shaken configurations, and the relativistic bandgap is smaller than $2.0 \mathrm{eV}$. Finally, they identified 10 compounds out of 248 candidates for possible solar cell applications with high-throughput screening, as shown in Fig. 1. After calculation, $\mathrm{AMgI}_{3}$ exhibited as a promising candidate with tunable bandgap between 0.9 and $1.7 \mathrm{eV}$ (1.7 eV for $\mathrm{CsMgI}_{3}, 1.5 \mathrm{eV}$ for $\mathrm{CH}_{3} \mathrm{NH}_{3} \mathrm{MgI}_{3}$ and $0.9 \mathrm{eV}$ for $\mathrm{CH}\left(\mathrm{NH}_{2}\right)_{2} \mathrm{MgI}_{3}$ ) and low electron effective mass, which is caused by exceptionally dispersive conduction bands [66] Therefore, Filip et al. deduced that the substitution of $\mathrm{Pb}$ by $\mathrm{Mg}$ was theoretically feasible to reduce the toxicity of metal halide perovskites without losing solar cell efficiency substantially. In subsequent research work, the researchers have also proved that $\mathrm{Cu}, \mathrm{Ag}, \mathrm{Bi}$ and $\mathrm{Sb}$ can also replace $\mathrm{Pb}$ to form perovskites (Fig. 1) [62-66].

In addition, it is possible to use valence state replacement to select suitable non-toxic elements by using the first-principles calculation [59, 64-75]. Usually, as shown in Fig. 2, we can replace the $\mathrm{Pb}$ element with either homovalent elements such as $\mathrm{Sn}, \mathrm{Ge}$, and $\mathrm{Cu}$, or heterovalent elements such as $\mathrm{Sb}$ and $\mathrm{Bi}$. To maintain the charge neutrality, the heterovalent replacement can be divided into three subcategories: cation splitting, mixed valence anion and ordered vacancy. Cation splitting is to form a double perovskite structure with a chemical formula of $\mathrm{A}_{2} \mathrm{~B}(\mathrm{I}) \mathrm{B}(\mathrm{III}) \mathrm{X}_{3}$ by splitting $\mathrm{Pb}$ into a combination of monovalent and trivalent cations. Introducing mixed valence anions is the case with a single valence cation but two valence anions, whose general chemical formula is $\mathrm{AB}(\mathrm{Ch}, \mathrm{X})_{3}$, where $\mathrm{Ch}$ represents a chalcogen element and $\mathrm{X}$ represents a halogen element. The perovskite can maintain electrical neutrality via forming ordered vacancies. Such substitutions can also be divided into two types: the $B$ (III) compounds with a formula of $A_{3} \square B(I I I) X_{9}$ and the $\mathrm{B}(\mathrm{IV})$ compounds with a formula of $\mathrm{A}_{2} \square \mathrm{B}(\mathrm{IV}) \mathrm{X}_{6}$. Here, the sign of $\square$ indicates vacancies. However, the formation of vacancies cuts the original 3D perovskite structure into a low dimension crystal structure, thereby reducing the electronic dimension and then affecting the optoelectronic performance [67].

Researchers have conducted a lot of theoretical studies on the $\mathrm{Pb}$ replacement with various elements, e.g., $\mathrm{Sn}, \mathrm{Ge}$, and Bi [57-62]. These theoretical studies reveal the advantages and disadvantages of different lead-free perovskite materials. In the substitution of homovalent elements, take Snbased perovskite $\mathrm{MASnI}_{3}$ as an example. In the process of studying the optoelectronic properties of $\mathrm{MASnI}_{3}$ using the $\mathrm{GW}+\mathrm{SOC}$ method, the researchers found that compared with $\mathrm{MAPbI}_{3}, \mathrm{MASnI}_{3}$ showed a stronger $s-p$ antibond coupling near the maximum value of the valence band (VBM) [68]. This phenomenon is due to the fact that the Sn $5 \mathrm{~s}$ lone pair is shallower and more active than the $\mathrm{Pb} 6$ s lone pair. 


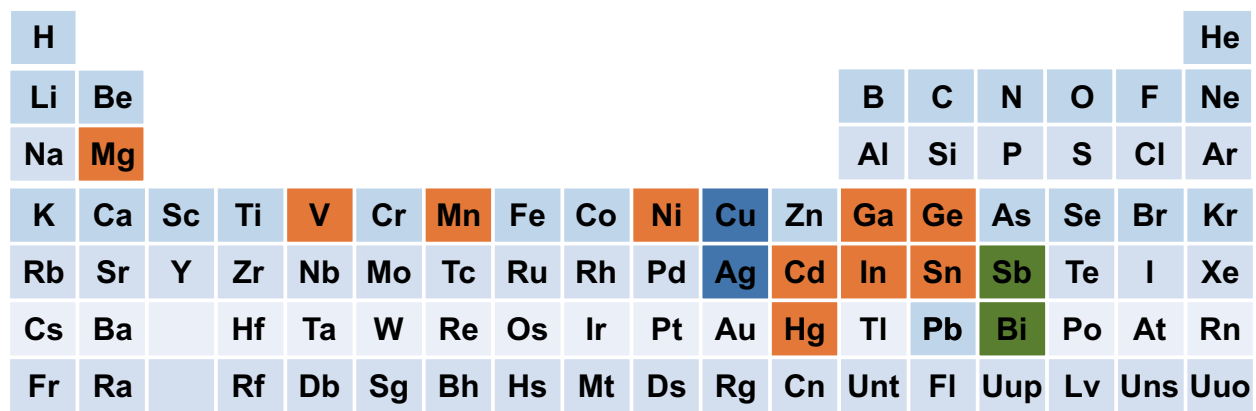

Fig. 1 Potential elements to substitute $\mathrm{Pb}$. The orange shading on the periodic table marks the screened elements by Filip et al. that can replace $\mathrm{Pb}$. The green shading of the VA group heterovalent elements and the blue shading of transition metal elements have also been calculated or proved to substitute $\mathrm{Pb}$. (Color figure online)

In addition, because of much weaker SOC, the $\mathrm{Sn} 5 \mathrm{p}$ orbital is shallower and less dispersive than the $\mathrm{Pb} 6 \mathrm{p}$ orbitals. As a result, compared to $\mathrm{MAPbI}_{3}$, the bandgap of $\mathrm{MASnI}_{3}$ is reduced. Unfortunately, defect calculations indicated that the high-energy Sn $5 s^{2}$ state makes the Sn-I bond easy to break, forming high-density $\mathrm{Sn}$ vacancies, resulting in an excessively high defect density $\left(>10^{17} \mathrm{~cm}^{-3}\right)$ in the Sn-based perovskite [68]. Similar to Sn-based perovskite, theoretical calculations by Ming et al. indicated that the Ge vacancy in Ge-based perovskite is the dominant defect with shallow transition levels and rather low formation enthalpy, which yields high-density holes, particularly at Ge-poor synthesis conditions [33]. At the same time, Sun et al. pointed out that the stability of $\mathrm{MAGeI}_{3}$ is better than that of $\mathrm{MASnI}_{3}$ based on the calculation of the formation energy of the compound. In addition, they calculated the electronic structure and improved bandgap results through the hybrid function HSE06 and the spin-orbit coupling [36]. The calculated band structure showed that the bottom of the conduction band and the top of the valence band are very dispersed, which corresponds to low electron/hole effective masses. These results mean that the replacement of $\mathrm{Pb}$ in perovskite by $\mathrm{Ge}$ is possible.

In the theoretical study of heterovalent replacement, using $\mathrm{Cs}_{3} \mathrm{Bi}_{2} \mathrm{I}_{9}$ as an example, the calculations using hybrid Heyd-Scuseria-Ernzerhof (HSE) functional with SOC indicated that $\mathrm{Cs}_{3} \mathrm{Bi}_{2} \mathrm{I}_{9}$ exhibits an indirect bandgap of $2.10 \mathrm{eV}$ from the $\Gamma$ point to the $\mathrm{K}$ point $[40,51]$. Moreover, the defect calculations by Ghosh et al. indicated that most of the defects in $\mathrm{Cs}_{3} \mathrm{Bi}_{2} \mathrm{I}_{9}$ that have low formation energies generate deep level states in the bandgap, which act as recombination centers [51]. Fortunately, this type of perovskite material has been calculated to own excellent thermodynamic stability.

In addition, the chalcogen-halogen hybrid perovskite $\mathrm{AB}(\mathrm{Ch}, \mathrm{X})_{3}$ was systematically studied by Sun et al. with $\mathrm{Ab}$ initio molecular dynamics, GGA functions and DFT-D3 van der Waals scheme [52]. According to relevant calculations, they predicted that $\mathrm{MABiSI}_{2}$ and $\mathrm{MABiSeI}_{2}$ have the best bandgap (1.3-1.4 eV) for solar energy collection materials. However, in later studies, Hong et al. combined DFT calculations and solid-state reactions to prove that all proposed $\mathrm{AB}(\mathrm{Ch}, \mathrm{X})_{3}$ perovskites are thermodynamically unstable [34]. Although this result is disappointing, it also reminds us that stability analysis is equally important in the theoretical calculation of the discovery of new lead-free perovskites.

Recently, the theoretical calculation has proved the 0D electronic dimension and larger bandgap of double perovskite $\mathrm{A}_{2} \mathrm{~B}(\mathrm{I}) \mathrm{B}(\mathrm{III}) \mathrm{X}_{6}$ with the use of alkali metal $\mathrm{B}$ (I) cation $[28,29]$. Actually, transition metal $\mathrm{B}(\mathrm{III})$ cations with multiple oxidation states and/or partially occupying $\mathrm{d}$ or $\mathrm{f}$ orbitals are not ideal for the design of photovoltaic absorbers because they may introduce deeper defect states and localized bandedges. Finally, regarding the screening of $\mathrm{X}$ anions, it is known that iodides and bromides have the potential of obtaining narrower bandgaps, but they are thermodynamically unstable, while fluorides and chlorides are relatively stable but usually have larger bandgaps [75-78]. The above theoretical research work undoubtedly played a guiding role in the application of new-type lead-free perovskite materials in perovskite solar cells, resulting in a rapid development of this field. 


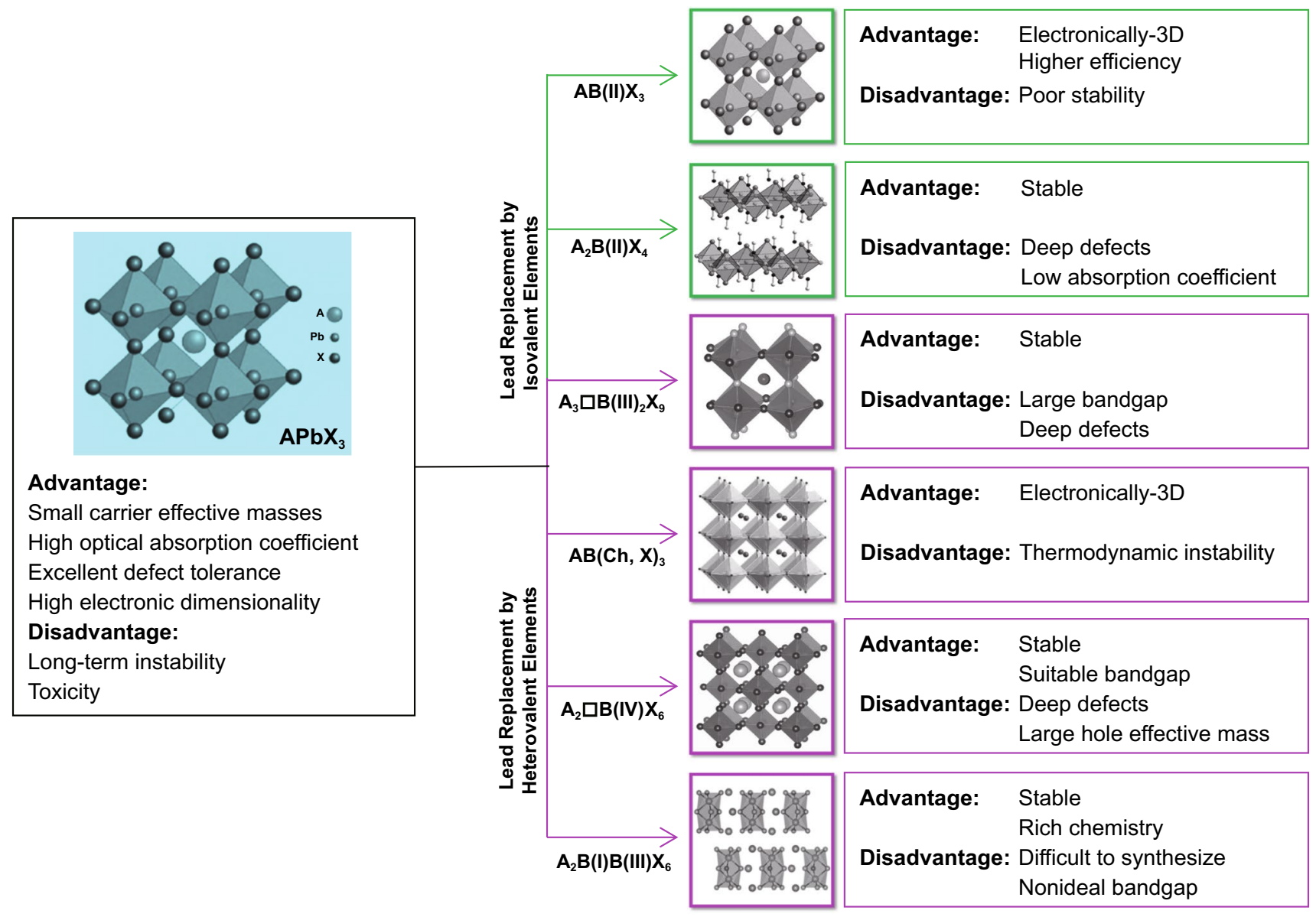

Fig. 2 Schematic illustration of the approaches and consequences of potential $\mathrm{Pb}$ replacement

\section{Experimental Investigation of Various Types of Lead-Free Hybrid Perovskites}

\subsection{Lead Replacement by Isovalent Elements}

\subsubsection{Sn-based Perovskite}

As a chemical element of $\mathrm{Pb}$ 's family (IVA group), $\mathrm{Sn}^{2+}$ possesses a homologous lone-pair s orbital and similar radius $(1.35 \AA)$ to $\mathrm{Pb}^{2+}(1.49 \AA)$; therefore, Sn-based perovskites show superior optoelectronic properties similar with $\mathrm{Pb}[68$, 69]. In addition, they also exhibit narrower optical bandgaps $(1.2-1.4 \mathrm{eV})$ and higher carrier mobilities than the $\mathrm{Pb}$ based counterparts. Moreover, due to intrinsic low toxicity of Sn, the Sn-based perovskite absorbers have become the important candidate for lead-free perovskites and gradually turned into the research hotspot in high-performance leadfree PSCs [68-70]. As a result, the Sn-based perovskites are more suitable for efficient single-junction solar cell applications. However, $\mathrm{Sn}^{2+}$ is unstable and easily oxidized to $\mathrm{Sn}^{4+}$. The formation of $\mathrm{Sn}^{4+}$ leads to p-type self-doping in Sn-based perovskites, which will generate numerous $\mathrm{Sn}$ vacancies with large background carrier density and rapid recombination of charge carriers [14], leading to serious performance degradation and poor reproducibility of PSCs using Sn-based perovskites as absorbers [26-30].

\subsubsection{3D Sn-based Perovskite}

(1) $\operatorname{MASn}(\mathrm{I}, \mathrm{Br})_{3}$

The first $\mathrm{MASnIBr}_{2}$ perovskite solar cell was realized with a PCE of 5.7\% in 2014 [70], whose PCE was then quickly refreshed to $6.4 \%$ by Zhao in the same year [71]. In 2015, Umari et al. investigated the optoelectronic properties of $\mathrm{MASnI}_{3}$ with a more advanced many-body perturbation 
theory involved with SOC [68]. They found $\mathrm{MASnI}_{3}$ exhibited a higher charge mobility of $10^{2}-10^{3} \mathrm{~cm}^{2} \mathrm{~V}^{-1} \mathrm{~s}^{-1}[68$, 69], a smaller direct bandgap of $1.1 \mathrm{eV}$ at the $\Gamma$ point and a higher absorption coefficient of $1.82 \times 10^{4} \mathrm{~cm}^{-1}$ in the visible region than $\mathrm{MAPbI}_{3}$, whose mobility, bandgap and absorption coefficient of the latter are $10-10^{2} \mathrm{~cm}^{2} \mathrm{~V}^{-1} \mathrm{~s}^{-1}$, $1.5 \mathrm{eV}$ and $1.80 \times 10^{4} \mathrm{~cm}^{-1}$, respectively $[56,70]$. These pretty results encouraged researchers to pay more attention to Sn-based perovskites. Interestingly, Sn-based perovskite materials exhibit n-type semiconductor characteristics when the internal $\mathrm{Sn}^{2+}$ content ratio is high, and p-type semiconductor characteristics when the $\mathrm{Sn}^{4+}$ content ratio is high. Until now plenty of efforts have been dedicated to development of high-performance and air-stable Sn-based perovskite solar cells $[33,34]$. Poor homogeneity and low coverage are critical issues to be solved in Sn-based perovskites, which usually lead to direct contact between the hole-transporting layer (HTL) and the electron-transporting layer (ETL), thus generating low PCEs [70, 72]. Here, film formation methods suitable for $\mathrm{Pb}$-based perovskites tend to become inefficient for Sn-based cases due to a faster crystallization rate of Snbased perovskites [73, 74]; for instance, one-step method with common solvent of $\mathrm{N}, \mathrm{N}$-dimethylformamide (DMF) or $\gamma$-butyrolactone (GBL) hardly realized smooth Sn-based perovskite films on flat substrates. Hao et al. studied the effect of solvent on the crystallization of $\mathrm{MASnI}_{3}$ perovskite film and found that adding dimethyl sulfoxide (DMSO) retarded the perovskite crystallization via the formation of a stable intermediate adduct $\mathrm{SnI}_{2} \cdot 3 \mathrm{DMSO}$ (Fig. 3a), the film formation speed of which was eventually controlled by the evaporation rate of DMSO. As the scanning electron microscopy (SEM) images shown in Fig. 3a-d, the morphologies of $\mathrm{MASnI}_{3}$ films with DMSO showed the highest coverage and pinhole-free property on mesoporous $\mathrm{TiO}_{2}$ [74]. Moreover, $\mathrm{N}$-methyl-2-pyrrolidone (NMP) was also investigated as a solvent to further understand the effect of the intermediate phase on the tin perovskite formation process. As a result, a high-quality, pinhole-free $\mathrm{CH}_{3} \mathrm{NH}_{3} \mathrm{SnI}_{3}$ films are achieved using NMP as solvents [73, 74].

Multi-step film formation method was another efficient approach to achieve high-quality Sn-based perovskite films $[19,56,75,76]$. Schlettwein et al. reported an approach combined thermal evaporation with solution process to fabricate $\mathrm{MASnI}_{3}$ perovskite films $[56,76]$. The $\mathrm{SnI}_{2}$ layers prepared by vapor thermal deposition could be converted to $\mathrm{MASnI}_{3}$ by sequentially spin coating MAI solution.
The as-acquired sizes of $\mathrm{MASnI}_{3}$ grains were over $200 \mathrm{~nm}$ (Fig. 3i-n) [75]. Moreover, the film morphologies were highly dependent on MAI concentrations. With the increasing concentration of MAI, larger and more uniform $\mathrm{MASnI}_{3}$ crystals were obtained (Fig. 3i-n). After 10-min thermal annealing, high-quality and dense films were achieved with significantly improved stability. The films remained stable after exposure to air for 90 min under both dark and light conditions.

In addition to the above approaches, Qi et al. invented a method to improve the quality of MASn(I, Br $)_{3}$-based perovskite film. They used co-evaporation and sequential evaporation methods to fabricate $\mathrm{MASnBr}_{3}$ perovskite films with $\mathrm{SnBr}_{2}$ and $\mathrm{MABr}$ as precursors [77]. For co-evaporation, they used a 4:1 MABr/SnBr 2 deposition ratio $\left(0.4: 0.1 \AA_{\mathrm{s}^{-1}}\right)$ to simultaneously evaporate the two materials, and finally produced a perovskite film with a thickness of about $400 \mathrm{~nm}$. However, solar cells based on this film only showed a low PCE of $0.35 \%$ (Fig. 4a). Such a low PCE was resulted from the oxidation of $\mathrm{Sn}^{2+}$ which restrained the generation of excitons, the carriers diffusion, and the final charge extraction. As a melioration, Qi et al. then employed sequential deposition method to decrease oxidation (Fig. 4b, c) and realized a relatively high PCE of $1.12 \%$ because oxidation can be significantly avoided by the top MABr layer [77]. The specific operation is first to deposit a 100-nm film of $\mathrm{SnBr}_{2}$, and then to precipitate a following $400-\mathrm{nm}$ film of $\mathrm{MABr}$ (Fig. 4d). The as-deposited sample was transferred from the vacuum system to a $\mathrm{N}_{2}$ glovebox for post-annealing so as to convert the two-layer sample to $\mathrm{MASnBr}_{3}$ perovskite. Therefore, sequential evaporation approach could be an efficient approach for fabricating high-quality Sn-based perovskites.

\section{(2) $\operatorname{CsSn}(\mathrm{I}, \mathrm{Br})_{3}$}

In 1974, Fisher et al. first synthesized and characterized allinorganic $\mathrm{CsSnX}_{3}$ compounds [78]. It was not until 2012, Shum et al. reported the application of $\mathrm{CsSnI}_{3}$ in perovskite solar cell [79]. A black thin film of $\mathrm{CsSnI}_{3}$ with a bandgap of $1.3 \mathrm{eV}$ was fabricated by alternate depositions of $\mathrm{SnCl}_{2}$ and CsI on a glass substrate followed by a thermal annealing process. The $\mathrm{CsSnI}_{3}$ perovskite solar cell exhibited a PCE of $0.9 \%$. Then in 2014, Mathews et al. reported a $\mathrm{CsSnI}_{3}$ PSC incorporated with $\mathrm{SnF}_{2}$ to reduce $\mathrm{Sn}$ vacancies and obtain a high photocurrent output [71]. The best PCE of $2.02 \%$ as well as a short-circuit current density $\left(J_{\mathrm{sc}}\right)$ of 

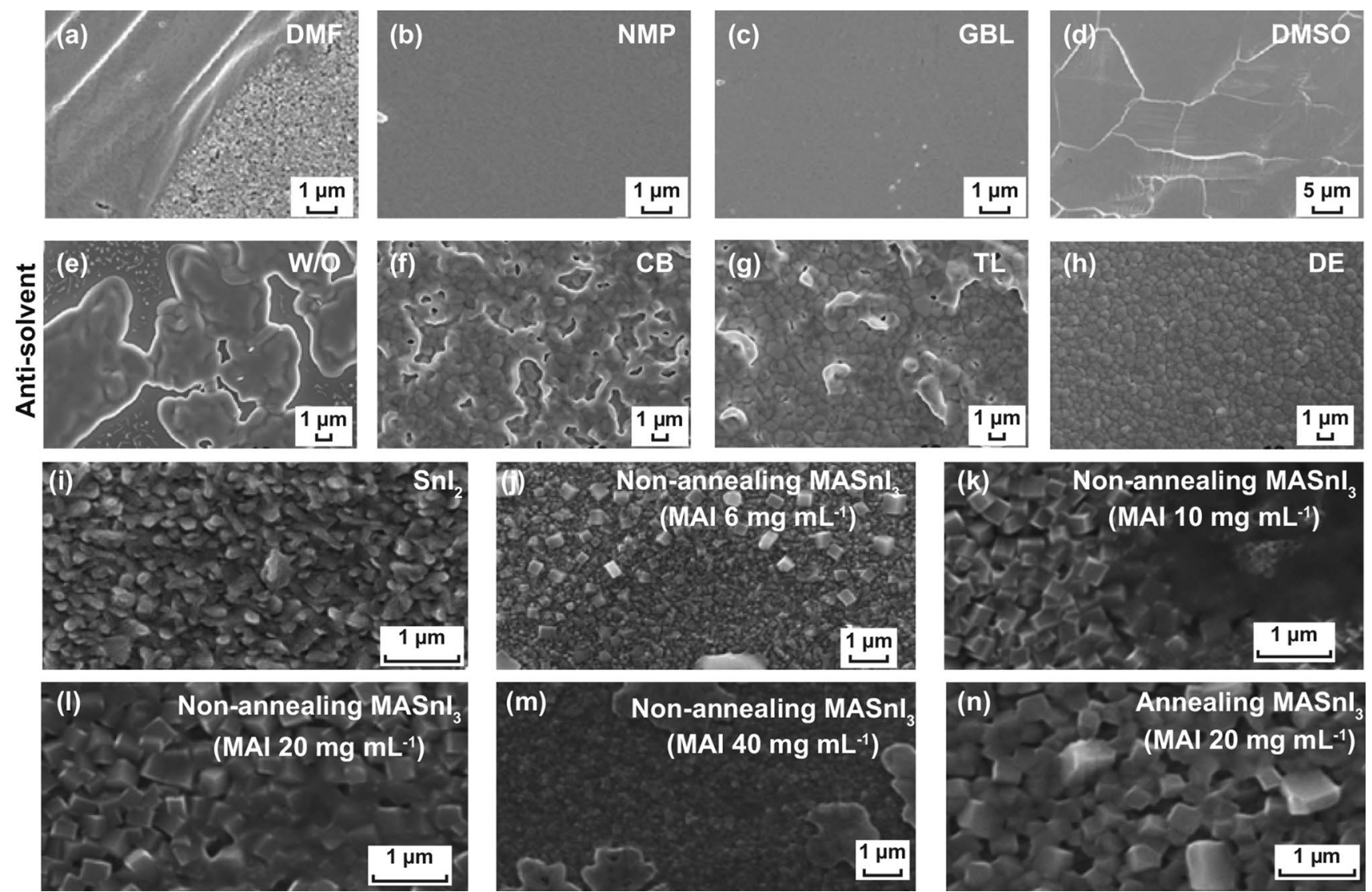

Fig. 3 SEM images for MASnI 3 layer on mesoporous $\mathrm{TiO}_{2}$ layer by using a DMF, b NMP, c GBL, and d DMSO solvents. Reproduced with permission from Ref. [74]. SEM images for $\mathrm{FASnI}_{3}$ films obtained from different antisolvent processes: e No dripping, $\mathbf{f} \mathrm{CB}, \mathbf{g}$ TL and $\mathbf{h}$ DE. Reproduced with permission from Ref. [96]. SEM images of $\mathbf{i}$ vapor deposited $100 \mathrm{~nm} \mathrm{SnI}$, non-annealing MASnI films with j 6, k 10, I 20 and $\mathbf{m} 40 \mathrm{mg} \mathrm{mL}^{-1}$ MAI precursor solution spin coated and $\mathbf{n}$ MASnI $_{3}$ films with $20 \mathrm{mg} \mathrm{mL}^{-1}$ MAI precursor solution spin coated followed by annealing at $80{ }^{\circ} \mathrm{C}$ for $10 \mathrm{~min}$. Reproduced with permission from Ref. [75]

$22.70 \mathrm{~mA} \mathrm{~cm}^{-2}$, an open-circuit voltage $\left(V_{\mathrm{oc}}\right)$ of $0.24 \mathrm{~V}$ and a fill factor (FF) of 0.37 was achieved with $20 \mathrm{~mol} \% \mathrm{SnF}_{2}$ (Fig. 4e). Moreover, the devices displayed less hysteresis than those counterparts without $\mathrm{SnF}_{2}$ (Fig. 4f). The incident photon-to-current conversion efficiency (IPCE) data clearly indicated that the onset was extended to $950 \mathrm{~nm}$, which corresponded to $1.3 \mathrm{eV}$ of bandgap (Fig. $4 \mathrm{~g}$ ). In 2015, a detailed study on $\mathrm{CsSnX}_{3}$ was done by Sabba et al. [80]. Their studies revealed that the $\mathrm{CsSnX}_{3}$ materials were semiconductors with a strong tendency to self-doping because the oxidation of $\mathrm{Sn}^{2+}$ to $\mathrm{Sn}^{4+}$ would generate hole carriers.

It is worth mentioning that $\mathrm{CsSnI}_{3}$ as a 3D p-type orthorhombic perovskite possesses a bandgap of $1.3 \mathrm{eV}$ [81], a low exciton binding energy of $18 \times 10^{-3} \mathrm{eV}$ [82], and a high optical absorption coefficient of $10^{4} \mathrm{~cm}^{-1}$ (comparable to $\mathrm{MAPbI}_{3}$ ) [83]. Therefore, it has the potential to be as a light absorber for lead-free PSCs. The biggest obstacle that hinders rapid development of $\mathrm{CsSnI}_{3}$ perovskite solar cell is the instability of $\mathrm{CsSnI}_{3}$ and the black phase $\mathrm{CsSnI}_{3}$ could be easily converted to yellow phase $\mathrm{CsSnI}_{3}$ in atmosphere due to its oxidization $[69,84]$. It was reported that the excessive $\mathrm{SnI}_{2}$ contributed to the improvements of efficiency and stability of $\mathrm{CsSnI}_{3}$-based PSCs [85]. The $\mathrm{CsSnI}_{3}$ films with low defect density and high surface coverage were prepared in a Sn-rich environment or at high temperature. The PCE of the device with a configuration of ITO/CuI/CsSnI $/$ fullerene/bathocuproine (BCP)/Al increased from 0.75 to $1.5 \%$ in the condition of $10 \mathrm{~mol} \%$ excessive $\mathrm{SnI}_{2}$, and $V_{\text {oc }}$ and FF remained good within 20 min under light stability. Although $J_{\text {sc }}$ quickly deteriorated by $10 \%$ in the first $50 \mathrm{~min}$, $J_{\text {sc }}$ 's decline rate slowed significantly. The excess of $\mathrm{SnI}_{2}$ located at the $\mathrm{CsSnI}_{3} / \mathrm{CuI}$ interface formed an interfacial 

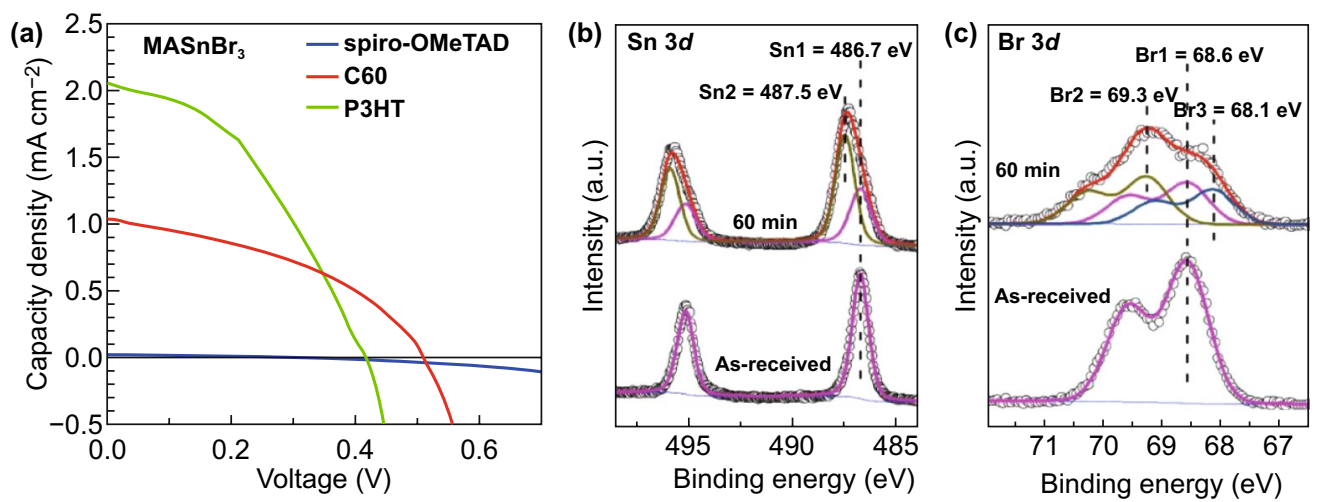

(d)

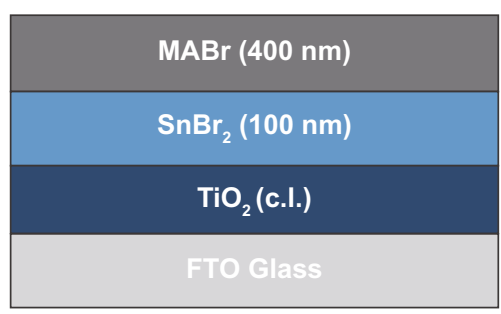

HTL

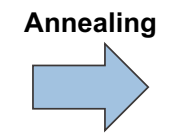

HTL spin casting
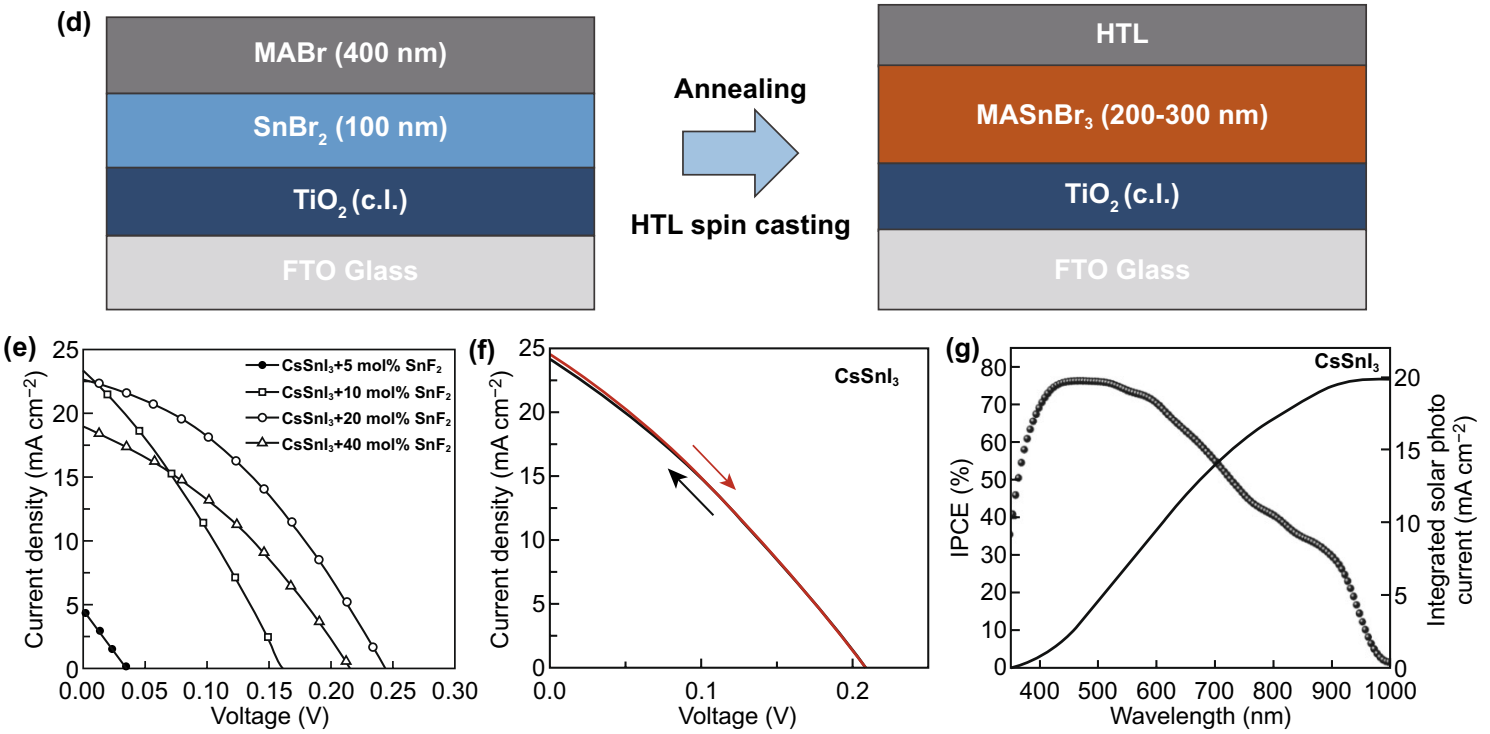

Fig. 4 a $J-V$ curves of co-evaporated $\mathrm{MASnBr}_{3}$ films with different hole transport materials. b Sn $3 \mathrm{~d}$ and $\mathbf{c}$ Br $3 \mathrm{~d}$ XPS results of MASnBr $\mathrm{M}_{3}$ films as-deposited and after $1 \mathrm{~h}$ stored in air. d Fabrication of sequential method for $\mathrm{MASnBr}_{3}$ films. Reproduced with permission from Ref. [77]. e $J-V$ curves of $\mathrm{CsSnI}_{3}$-based PSCs incorporated with different ratios of $\mathrm{SnF}_{2}$. f $J-V$ curves of $20 \mathrm{~mol}_{\%} \mathrm{SnF}_{2}$-doped CsSnI 3 devices showing no hysteresis. $\mathbf{g}$ IPCE spectrum for $20 \mathrm{~mol} \% \mathrm{SnF}_{2}$-doped $\mathrm{CsSnI}_{3}$ devices. Reproduced with permission from Ref. [71]

dipole which acted as a hole-transporting layer from $\mathrm{CsSnI}_{3}$ to $\mathrm{CuI}$ owing to a favorable vacuum level shift.

After that, some additives have been introduced into precursors to further improve film quality of $\mathrm{CsSn}(\mathrm{I}, \mathrm{Br})_{3}$ perovskites, which have significantly promoted the performances of $\mathrm{CsSn}(\mathrm{I}, \mathrm{Br})_{3}$ perovskite solar cells [86-90]. Marshall et al. systematically investigated $\mathrm{CsSnI}_{3}$ perovskite films with different $\mathrm{Sn}$ halide additives, such as $\mathrm{SnF}_{2}, \mathrm{SnCl}_{2}$, $\mathrm{SnI}_{2}$, and $\mathrm{SnBr}_{2}$ [90]. The films adding $\mathrm{SnCl}_{2}$ additive had the highest pinhole density and the HTL-free devices with $10 \mathrm{~mol} \% \mathrm{SnCl}_{2}$ achieved the highest FF and PCE. Such results were mainly attributed to the formation of ultrathin hole-selective layer of $\mathrm{SnCl}_{2}$ at the ITO/CsSnI ${ }_{3}$ interface.
On this basis, doping $\mathrm{Br}$ into $\mathrm{CsSnI}_{3}$ was also proposed. It is noteworthy that the $\mathrm{Br}$-doped $\mathrm{CsSnI}_{3-\mathrm{x}} \mathrm{Br}_{\mathrm{x}}$ perovskites showed a much higher FF compared to $\mathrm{CsSnI}_{3}$ due to the appearance of the negligible overlayer of $\mathrm{CsSnI}_{3-\mathrm{x}} \mathrm{Br}_{\mathrm{x}}$ [80]. The crystal structure was thus changed from orthorhombic $\left(\mathrm{CsSnI}_{3}\right)$ to cubic $\left(\mathrm{CsSnBr}_{3}\right)$ with the increase in $\mathrm{Br}$ component. The onset of optical bandgap edge was also altered from $1.27 \mathrm{eV}\left(\mathrm{CsSnI}_{3}\right)$ to $1.37,1.65$, and $1.75 \mathrm{eV}$ for $\mathrm{CsSnI}_{2} \mathrm{Br}, \mathrm{CsSnIBr}_{2}$ and $\mathrm{CsSnBr}_{3}$, respectively (Fig. 5). Notably, $\mathrm{CsSnI}_{2} \mathrm{Br}, \mathrm{CsSnIBr}_{2}$ and $\mathrm{CsSnBr}_{3}$ were suitable for solar cell applications due to their excellent thermal and air stabilities (Table 1) [80]. 


\section{(3) $\mathrm{FASn}(\mathrm{I}, \mathrm{Br})_{3}$}

Compared to $\mathrm{MA}^{+}$and $\mathrm{Cs}^{+}, \mathrm{FA}^{+}$is another extensively investigated organic cation with a relatively larger ionic radius. $\mathrm{FASnI}_{3}$ is a $3 \mathrm{D}$ perovskite with a bandgap of $1.41 \mathrm{eV}$, which is slightly wider than $\operatorname{MASnI}_{3}(1.30 \mathrm{eV})$ and $\mathrm{CsSnI}_{3}(1.30 \mathrm{eV})$ [37] but narrower than Pb-based perovskites $(\sim 1.5 \mathrm{eV})$ [69]. Moreover, the bandgaps of FASnX can be tuned with different halides, e.g., 1.68 and $2.4 \mathrm{eV}$ for $\mathrm{FASnI}_{2} \mathrm{Br}$ and $\mathrm{FASnBr}_{3}[78,91]$. The $\mathrm{FASnI}_{3}$ material exhibits a threshold charge-carrier density of $8 \times 10^{17} \mathrm{~cm}^{-3}$ and a charge-carrier mobility of $22 \mathrm{~cm}^{2} \mathrm{~V}^{-1} \mathrm{~s}^{-1}$ [92]. In addition, $\mathrm{FASnI}_{3}$ has a similar thermal stability but a lower conductivity with respect to $\mathrm{MASnI}_{3}$ [93].

Synthesis and characterization of cubic $\mathrm{FASnI}_{3}$ perovskite were first investigated in 1997 [94]. Until now, most highperformance Sn-based PSCs are mainly based on $\mathrm{FASnI}_{3}$ materials [95-97] because of their better air stability. This is because the trap density of $\mathrm{FASnI}_{3}$ (n-type semiconductor) is as low as $\sim 10^{11} \mathrm{~cm}^{-3}$, preventing water and oxygen from entering into the $\mathrm{FASnI}_{3}$ crystal, which has been experimentally proved by Wang et al. [98]. Therefore, the air stability of the $\mathrm{FASnI}_{3}$ crystal is higher than $\mathrm{MASnI}_{3}$ one.

After the first successful incorporation of $\mathrm{SnF}_{2}$ into $\mathrm{CsSnI}_{3}$, Mathews group further verified similar effect of $\mathrm{SnF}_{2}$ additive on performance enhancements of FASnI ${ }_{3}$-based solar cells. $\mathrm{SnF}_{2}$ additive played a similar role in preventing oxidation of $\mathrm{Sn}^{2+}$ to $\mathrm{Sn}^{4+}$ and reducing background carrier density, and the $\mathrm{FASnI}_{3}$ PSC with $20 \%$ $\mathrm{SnF}_{2}$ finally achieved a PCE of $2.1 \%$ [99].

However, it has been proven that a higher amount of $\mathrm{SnF}_{2}$ induced severe phase separation in the $\mathrm{FASnI}_{3}$ perovskites films and thus led to performance degradation of solar cells. To solve this issue, Seok et al. introduced pyrazine into DMF/DMSO mixed precursor solution to fabricate highquality $\mathrm{FASnI}_{3}$ films with high coverage and smooth surface [87]. Since the $\mathrm{N}$ atoms in pyrazine can accept lone pairs electrons, pyrazine doping can remarkably restrict the phase separation and effectively reduce $S n$ vacancies [84], with which a dense, smooth, and pinhole-free $\mathrm{FASnI}_{3}$ perovskite layer and a pretty good PCE of $4.8 \%$ were achieved with high reproducibility. Furthermore, the encapsulated solar cells exhibited good long-term stability, remaining $98 \%$ of the initial PCE for over 10-day storage under ambient condition. It is worth mentioning that, in addition to $\mathrm{SnF}_{2}$ and pyrazine, hydrazine vapor, hydrazine iodide [69], and Sn powder [100] have also been used as antioxidants in Sn-based perovskites in later work. The application of these antioxidants has a similar effect with $\mathrm{SnF}_{2}$, that is, to improve the stability and efficiency of Sn-based PSCs by reducing Sn vacancies and background carriers in $\mathrm{Sn}$ perovskites.

In addition, some other special materials have also been served as additives to improve the film quality of perovskites and hinder the oxidation of $\mathrm{Sn}^{2+}$. For instance, Chen et al. used the bidentate ligand 8-hydroxyquinoline (8HQ) as an additive in the perovskite [101]. The $\mathrm{N}$ and $\mathrm{O}$ atoms in the 8HQ can simultaneously coordinate with $\mathrm{Sn}^{2+}$ and greatly inhibit the oxidation of $\mathrm{Sn}^{2+}$. Meantime, the formation of complex also improved the quality of $\mathrm{FASnI}_{3}$ film and reduced defect state-induced non-radiative recombination. Recently, ammonium hypophosphite was introduced into the $\mathrm{FASnI}_{3}$ perovskite precursor by Yan et al. to suppress the oxidation of $\mathrm{Sn}^{2+}$ and assist the growth of perovskite grains, resulting in improved perovskite film

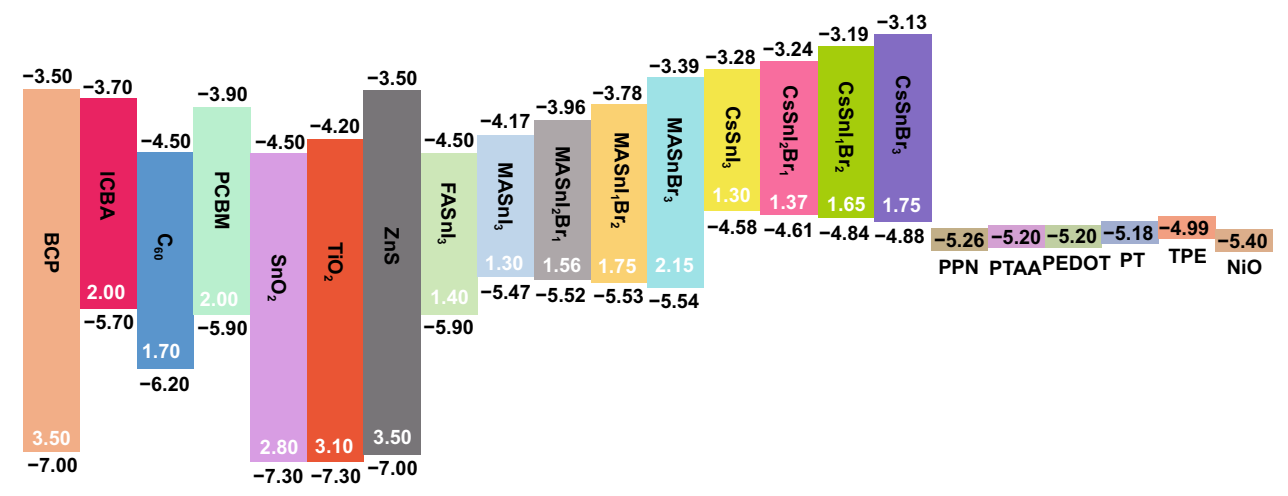

Fig. 5 Energy diagram of common Sn-based perovskites, ETLs and HTLs. Unit: eV 


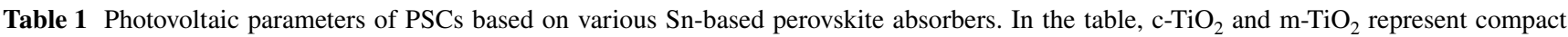
and mesoporous $\mathrm{TiO}_{2}$ layer, respectively

\begin{tabular}{|c|c|c|c|c|c|c|c|}
\hline Preparation process & Absorber & Device architecture & $V_{\mathrm{oc}}(\mathrm{V})$ & $J_{\mathrm{sc}}\left(\mathrm{mA} \mathrm{cm}^{-2}\right)$ & $\mathrm{FF}(\%)$ & PCE $(\%)$ & References \\
\hline One-step & $\mathrm{MASnI}_{3}$ & $\begin{array}{l}\mathrm{c}-\mathrm{TiO}_{2} / \mathrm{m}_{-} \mathrm{TiO}_{2} / \text { perovskite/ } \\
\text { spiro-MeOTAD/Au }\end{array}$ & 0.68 & 16.30 & 48 & 5.23 & {$[70]$} \\
\hline One-step & $\mathrm{MASnI}_{2} \mathrm{Br}$ & $\begin{array}{l}\text { c- } \mathrm{TiO}_{2} / \mathrm{m}-\mathrm{TiO}_{2} / \text { perovskite/ } \\
\text { spiro-MeOTAD/Au }\end{array}$ & 0.77 & 14.38 & 50 & 5.48 & {$[70]$} \\
\hline One-step & $\mathrm{MASnIBr}_{2}$ & $\begin{array}{l}\text { c- } \mathrm{TiO}_{2} / \mathrm{m}^{-\mathrm{TiO}_{2}} / \text { perovskite/ } \\
\text { spiro-MeOTAD/Au }\end{array}$ & 0.82 & 12.30 & 57 & 5.73 & {$[70]$} \\
\hline One-step & $\mathrm{MASnBr}_{3}$ & $\begin{array}{l}\text { c- }-\mathrm{TiO}_{2} / \mathrm{m}^{-\mathrm{TiO}_{2}} / \text { perovskite/ } \\
\text { spiro-MeOTAD/Au }\end{array}$ & 0.88 & 8.26 & 59 & 4.27 & {$[70]$} \\
\hline One-step & $\mathrm{MASnI}_{3}$ & $\begin{array}{l}\mathrm{c}-\mathrm{TiO}_{2} / \mathrm{m}_{-} \mathrm{TiO}_{2} / \text { perovskite/ } \\
\text { spiro-MeOTAD/Au }\end{array}$ & 0.716 & 15.18 & 50.07 & 5.44 & {$[135]$} \\
\hline One-step & $\mathrm{MASnI}_{3}$ & $\begin{array}{l}\text { c- } \mathrm{TiO}_{2} / \mathrm{m}^{-\mathrm{TiO}_{2}} / \text { perovskite/ } \\
\text { spiro-MeOTAD/Au }\end{array}$ & 0.88 & 16.8 & 42 & 6.4 & [82] \\
\hline One-step & $\mathrm{MASnI}_{3}$ & $\begin{array}{l}\text { c- } \mathrm{TiO}_{2} / \mathrm{m}^{-\mathrm{TiO}_{2}} / \text { perovskite/ } \\
\text { spiro-MeOTAD/Au }\end{array}$ & 0.79 & 13.40 & 52 & 5.49 & {$[124]$} \\
\hline One-step & $\mathrm{CsSnI}_{3}+20 \% \mathrm{SnF}_{2}$ & $\begin{array}{l}\mathrm{c}-\mathrm{TiO}_{2} / \mathrm{m}^{-\mathrm{TiO}_{2}} / \text { perovskite/ } \\
\text { spiro-MeOTAD/Au }\end{array}$ & 0.201 & 27.67 & 29 & 1.66 & {$[91]$} \\
\hline One-step & $\mathrm{CsSnI}_{2} \mathrm{Br}+20 \% \mathrm{SnF}_{2}$ & $\begin{array}{l}\text { c- }-\mathrm{TiO}_{2} / \mathrm{m}^{-\mathrm{TiO}_{2}} / \text { perovskite/ } \\
\text { spiro-MeOTAD/Au }\end{array}$ & 0.289 & 15.06 & 38 & 1.67 & [91] \\
\hline One-step & $\mathrm{CsSnIBr}_{2}+20 \% \mathrm{SnF}_{2}$ & $\begin{array}{l}\text { c-TiO } / 2 / \mathrm{m}^{-\mathrm{TiO}_{2}} / \text { perovskite/ } \\
\text { spiro-MeOTAD/Au }\end{array}$ & 0.311 & 11.57 & 43 & 1.56 & {$[91]$} \\
\hline One-step & $\mathrm{CsSnI}_{2.9} \mathrm{Br}_{0.1}+20 \% \mathrm{SnF}_{2}$ & $\begin{array}{l}\text { c- } \mathrm{TiO}_{2} / \mathrm{m}-\mathrm{TiO}_{2} / \text { perovskite/ } \\
\text { spiro-MeOTAD/Au }\end{array}$ & 0.222 & 24.16 & 33 & 1.76 & [91] \\
\hline One-step & $\mathrm{FASnI}_{3}+20 \% \mathrm{SnF}_{2}$ & $\begin{array}{l}\text { c- } \mathrm{TiO}_{2} / \mathrm{m}_{-} \mathrm{TiO}_{2} / \text { perovskite/ } \\
\text { spiro-MeOTAD/Au }\end{array}$ & 0.238 & 24.45 & 36 & 2.10 & [99] \\
\hline One-step & $\mathrm{CsSnBr}_{3}+20 \% \mathrm{SnF}_{2}$ & $\begin{array}{l}\text { c-TiO } / 2 / \mathrm{m}^{-\mathrm{TiO}_{2}} / \text { perovskite/ } \\
\text { spiro-MeOTAD/Au }\end{array}$ & 0.42 & 9.1 & 57 & 2.17 & [89] \\
\hline One-step & $\mathrm{MASnIBr}_{2}$ & $\begin{array}{l}\mathrm{c}-\mathrm{TiO}_{2} / \mathrm{m}-\mathrm{TiO}_{2} / \text { perovskite/ } \\
\text { spiro-MeOTAD/Au }\end{array}$ & 0.69 & 15.9 & 49 & 5.36 & [134] \\
\hline One-step & $\mathrm{MASnI}_{3}+20 \% \mathrm{SnF}_{2}$ & $\begin{array}{l}\mathrm{c}-\mathrm{TiO}_{2} / \mathrm{m}-\mathrm{TiO}_{2} / \text { perovskite/ } \\
\text { PTAA/Au }\end{array}$ & 0.25 & 26.1 & 30 & 1.94 & [137] \\
\hline One-step & $\{\mathrm{en}\} \mathrm{FASnI}_{3}+15 \% \mathrm{SnF}_{2}$ & $\begin{array}{l}\text { c-TiO }{ }_{2} / \mathrm{m}-\mathrm{TiO}_{2} / \text { perovskite/ } \\
\text { PTAA/Au }\end{array}$ & 0.48 & 22.54 & 65.96 & 7.14 & {$[81]$} \\
\hline One-step & $\{$ en $\} \mathrm{FASnI}_{3}+15 \% \mathrm{SnF}_{2}$ & $\begin{array}{l}\text { c-TiO }{ }_{2} / \mathrm{m}-\mathrm{TiO}_{2} / \text { perovskite/ } \\
\text { PTAA/Au }\end{array}$ & 0.46 & 22.54 & 69.74 & 7.23 & [138] \\
\hline One-step & $\{\mathrm{PN}\} \mathrm{FASnI}_{3}+15 \% \mathrm{SnF}_{2}$ & $\begin{array}{l}\mathrm{c}-\mathrm{TiO}_{2} / \mathrm{m}-\mathrm{TiO}_{2} / \text { perovskite/ } \\
\mathrm{TPE} / \mathrm{Au}\end{array}$ & 0.44 & 22.15 & 60.67 & 5.85 & [109] \\
\hline One-step & $\{\mathrm{TN}\} \mathrm{FASnI}_{3}+15 \% \mathrm{SnF}_{2}$ & $\begin{array}{l}\text { c- } \mathrm{TiO}_{2} / \mathrm{m}-\mathrm{TiO}_{2} / \text { perovskite/ } \\
\text { PTAA/Au }\end{array}$ & 0.40 & 22.72 & 61.04 & 5.53 & [109] \\
\hline One-step & $\{$ en $\} \mathrm{MASnI}_{3}+15 \% \mathrm{SnF}_{2}$ & $\begin{array}{l}\text { c- } \mathrm{TiO}_{2} / \mathrm{m}-\mathrm{TiO}_{2} / \text { perovskite/ } \\
\text { PTAA/Au }\end{array}$ & 0.43 & 24.28 & 63.72 & 6.63 & [108] \\
\hline One-step & $\mathrm{MASnBr}_{3}$ & $\begin{array}{l}\mathrm{c}-\mathrm{TiO}_{2} / \mathrm{m}-\mathrm{TiO}_{2} / \text { perovskite/ } \\
\text { PTAA/Au }\end{array}$ & 0.307 & 1.22 & 36.8 & 0.14 & [139] \\
\hline One-step & $\mathrm{CsSnI}_{3}+20 \% \mathrm{SnF}_{2}$ & $\begin{array}{l}\mathrm{c}-\mathrm{TiO}_{2} / \mathrm{m}^{-\mathrm{TiO}_{2}} / \text { perovskite } / m \text { - } \\
\text { MTDATA } / \mathrm{Au}\end{array}$ & 0.24 & 22.70 & 37 & 2.02 & {$[88]$} \\
\hline One-step & $\mathrm{MASnCl}_{3}$ & $\mathrm{c}-\mathrm{TiO}_{2} /$ perovskite/CuSCN/Ag & 0.576 & 12.89 & 55 & 3.41 & {$[140]$} \\
\hline One-step & $\mathrm{CsSnI}_{3}$ & $\mathrm{NiO} /$ perovskite/PCBM/Al & 0.52 & 10.21 & 62.5 & 3.31 & [141] \\
\hline One-step & $\mathrm{CsSnI}_{3}$ & $\begin{array}{l}\text { c- } \mathrm{TiO}_{2} / \text { perovskite/spiro-MeO- } \\
\mathrm{TAD} / \mathrm{Au}\end{array}$ & 0.48 & 8.11 & 19.8 & 0.77 & {$[141]$} \\
\hline One-step & $\mathrm{HEA}_{0.4} \mathrm{FA}_{0.6} \mathrm{SnI}_{3}$ & $\begin{array}{l}\text { c- } \mathrm{TiO}_{2} / \mathrm{m}-\mathrm{TiO}_{2} / \text { perovskite/ } \\
\mathrm{Al}_{2} \mathrm{O}_{3} / \mathrm{C}\end{array}$ & 0.37 & 18.52 & 56.2 & 3.9 & [118] \\
\hline One-step & $\mathrm{GA}_{0.2} \mathrm{FA}_{0.78} \mathrm{SnI}_{3}+1 \% \mathrm{EDAI}_{2}$ & $\begin{array}{l}\text { PEDOT:PSS/perovskite/C } 60 \\
\text { BCP/Ag }\end{array}$ & 0.61 & 21.2 & 72 & 9.6 & [119] \\
\hline
\end{tabular}


Table 1 (continued)

\begin{tabular}{|c|c|c|c|c|c|c|c|}
\hline Preparation process & Absorber & Device architecture & $V_{\mathrm{oc}}(\mathrm{V})$ & $J_{\mathrm{sc}}\left(\mathrm{mA} \mathrm{cm}^{-2}\right)$ & $\mathrm{FF}(\%)$ & PCE $(\%)$ & References \\
\hline One-step & $\mathrm{FASnI}_{3}$ & $\begin{array}{l}\text { PEDOT:PSS/perovskite/ } \\
\text { PCBM/BCP/Ag }\end{array}$ & 0.49 & 22.24 & 65.19 & 7.15 & {$[101]$} \\
\hline One-step & $\mathrm{FASnI}_{3}$ & $\begin{array}{l}\mathrm{SnO}_{2} / \text { perovskite/PCBM/BCP/ } \\
\mathrm{Ag}\end{array}$ & 0.55 & 19.39 & 68.82 & 7.34 & [102] \\
\hline One-step & $\mathrm{FASnI}_{3}$ & $\begin{array}{l}\text { PEDOT:PSS/perovskite/C }{ }_{60} / \\
\text { BCP/Ag }\end{array}$ & 0.63 & 21.6 & 74.7 & 10.17 & [103] \\
\hline One-step & $\mathrm{FASnI}_{3}$ & $\begin{array}{l}\text { PEDOT:PSS/perovskite/C } 60 \\
\text { BCP/Ag }\end{array}$ & 0.628 & 22.25 & 74.2 & 10.37 & [104] \\
\hline One-step & $\mathrm{PEA}_{\mathrm{x}} \mathrm{FA}_{1-\mathrm{x}} \mathrm{SnI}_{3}+\mathrm{NH}_{4} \mathrm{SCN}$ & $\begin{array}{l}\text { PEDOT:PSS/perovskite/ } \\
\text { ICBA/BCP/Ag }\end{array}$ & 0.94 & 17.4 & 75 & 12.4 & {$[135]$} \\
\hline One-step & $\mathrm{FASnI}_{3}+5 \% \mathrm{PHCl}$ & $\begin{array}{l}\text { PEDOT:PSS/perovskite/C } 60 \\
\text { BCP/Ag }\end{array}$ & 0.76 & 23.5 & 64 & 11.4 & [136] \\
\hline Hot-casting & $\mathrm{BA}_{2} \mathrm{MA}_{3} \mathrm{Sn}_{4} \mathrm{I}_{13}+100 \% \mathrm{SnF}_{2}$ & $\begin{array}{l}\mathrm{c}-\mathrm{TiO}_{2} / \mathrm{m}-\mathrm{TiO}_{2} / \text { perovskite/ } \\
\text { PTAA } / \mathrm{Au}\end{array}$ & 0.229 & 24.1 & 45.7 & 2.53 & {$[120]$} \\
\hline Hot-casting & $\mathrm{MASnI}_{3}+20 \% \mathrm{SnF}_{2} /$ hydrazine & $\begin{array}{l}\mathrm{c}-\mathrm{TiO}_{2} / \mathrm{m}-\mathrm{TiO}_{2} / \text { perovskite/ } \\
\text { PTAA/Au }\end{array}$ & 0.378 & 19.92 & 51.73 & 3.89 & [142] \\
\hline Hot-casting & $\mathrm{CsSnI}_{3}+20 \% \mathrm{SnF}_{2} /$ hydrazine & $\begin{array}{l}\mathrm{c}-\mathrm{TiO}_{2} / \mathrm{m}-\mathrm{TiO}_{2} / \text { perovskite/ } \\
\text { PTAA/Au }\end{array}$ & 0.170 & 30.75 & 34.88 & 1.83 & {$[142]$} \\
\hline Hot-casting & $\mathrm{CsSnBr}_{3}+20 \% \mathrm{SnF}_{2}$ & $\begin{array}{l}\mathrm{c}-\mathrm{TiO}_{2} / \mathrm{m}-\mathrm{TiO}_{2} / \text { perovskite/ } \\
\text { PTAA/Au }\end{array}$ & 0.367 & 13.96 & 59.36 & 3.04 & {$[142]$} \\
\hline Hot-casting & $\mathrm{MASnI}_{3}$ & PEDOT/perovskite/PCBM/Al & 0.595 & 17.8 & 29.8 & 3.2 & [143] \\
\hline Vapor-assisted & $\mathrm{MASnI}_{3}$ & $\begin{array}{l}\mathrm{c}-\mathrm{TiO}_{2} / \mathrm{m}-\mathrm{TiO}_{2} / \text { perovskite/ } \\
\text { PTAA } / \mathrm{Au}\end{array}$ & 0.273 & 17.36 & 39.1 & 1.86 & [143] \\
\hline Vapor-assisted & $\mathrm{MASnI}_{3-\mathrm{x}} \mathrm{Br}_{\mathrm{x}}$ & $\begin{array}{l}\mathrm{c}-\mathrm{TiO}_{2} / \mathrm{m}^{-\mathrm{TiO}_{2}} / \text { perovskite/ } \\
\text { PTAA } / \mathrm{Au}\end{array}$ & 0.452 & 5.02 & 48.3 & 1.10 & [139] \\
\hline Solvent-engineering & $\mathrm{FASnI}_{3}+10 \% \mathrm{SnF}_{2}+$ pyrazine & $\begin{array}{l}\text { c- } \mathrm{TiO}_{2} / \mathrm{m}^{-\mathrm{TiO}_{2}} / \text { perovskite/ } \\
\text { spiro-MeOTAD/Au }\end{array}$ & 0.32 & 23.7 & 63 & 4.8 & [71] \\
\hline Solvent-engineering & $\mathrm{FASnI}_{3}+20 \% \mathrm{SnF}_{2}$ & $\begin{array}{l}\text { c- } \mathrm{TiO}_{2} / \mathrm{m}-\mathrm{TiO}_{2} / \text { perovskite/ } \\
\text { spiro-MeOTAD/Au }\end{array}$ & 0.38 & 23.09 & 60.01 & 5.27 & [97] \\
\hline Solvent-engineering & $\mathrm{MASnI}_{3}+20 \% \mathrm{SnF}_{2}$ & $\begin{array}{l}\mathrm{c}-\mathrm{TiO}_{2} / \mathrm{m}-\mathrm{TiO}_{2} / \text { perovskite/ } \\
\text { spiro-MeOTAD/Au }\end{array}$ & 0.232 & 26.0 & 38.6 & 2.33 & [134] \\
\hline Solvent-engineering & $\mathrm{FASnI}_{3}+10 \% \mathrm{SnF}_{2}$ & $\begin{array}{l}\text { PEDOT:PSS/perovskite/C } 60 / \\
\text { BCP/Ag }\end{array}$ & 0.47 & 22.07 & 60.67 & 6.22 & [96] \\
\hline Solvent-engineering & $\begin{array}{l}\mathrm{FASnI}_{3}+20 \% \text { PEAI }+10 \% \\
\underset{\mathrm{SnF}_{2}}{ }\end{array}$ & $\mathrm{NiO} /$ perovskite/PCBM/Al & 0.59 & 14.44 & 69 & 5.94 & [127] \\
\hline Solvent-engineering & $\begin{array}{l}(\mathrm{FA})_{0.75}(\mathrm{MA})_{0.25} \mathrm{SnI}_{3}+10 \% \\
\mathrm{SnF}_{2}\end{array}$ & $\begin{array}{l}\text { PEDOT:PSS/perovskite/C } 60 / \\
\text { BCP/Ag }\end{array}$ & 0.61 & 21.2 & 62.7 & 8.12 & {$[80]$} \\
\hline Solvent-engineering & $\begin{array}{l}(\mathrm{FA})_{0.5}(\mathrm{MA})_{0.5} \mathrm{SnI}_{3}+10 \% \\
\mathrm{SnF}_{2}\end{array}$ & $\begin{array}{l}\text { PEDOT:PSS/perovskite/C } 60 \\
\text { BCP/Ag }\end{array}$ & 0.53 & 21.3 & 52.4 & 5.92 & {$[80]$} \\
\hline Solvent-engineering & $\begin{array}{l}(\mathrm{FA})_{0.25}(\mathrm{MA})_{0.75} \mathrm{SnI}_{3}+10 \% \\
\mathrm{SnF}_{2}\end{array}$ & $\begin{array}{l}\text { PEDOT:PSS/perovskite/C } 60 \\
\text { BCP/Ag }\end{array}$ & 0.48 & 20.7 & 45.2 & 4.49 & {$[80]$} \\
\hline Solvent-engineering & $\mathrm{FASnI}_{3}+10 \% \mathrm{SnF}_{2}$ & $\begin{array}{l}\text { PEDOT:PSS/perovskite/C } 60 / \\
\text { BCP/Ag }\end{array}$ & 0.48 & 21.3 & 64.6 & 6.60 & [80] \\
\hline Solvent-engineering & $\mathrm{MASnI}_{3}+10 \% \mathrm{SnF}_{2}$ & $\begin{array}{l}\text { PEDOT:PSS/perovskite/C } 60 \\
\text { BCP/Ag }\end{array}$ & 0.46 & 21.4 & 42.7 & 4.29 & {$[80]$} \\
\hline Solvent-engineering & $\begin{array}{l}\text { 0.92FASnI } \\
\mathrm{SnF}_{2}\end{array}$ & $\begin{array}{l}\text { PEDOT:PSS/perovskite/C } 60 \\
\text { BCP/Ag }\end{array}$ & 0.525 & 24.1 & 71 & 9.0 & [144] \\
\hline Solvent-engineering & $\mathrm{FASnI}_{2} \mathrm{Br}$ & $\begin{array}{l}\text { PEDOT:PSS/perovskite/C } 60 \\
\mathrm{Ca} / \mathrm{Al}\end{array}$ & 0.467 & 6.82 & 54 & 1.72 & [89] \\
\hline Solvent-engineering & $\mathrm{MA}_{0.9} \mathrm{Cs}_{0.1} \mathrm{SnI}_{3}$ & $\begin{array}{l}\text { PEDOT:PSS/perovskite/ } \\
\text { PCBM/Bis- } \mathrm{C}_{60} / \mathrm{Ag}\end{array}$ & 0.20 & 4.53 & 36.4 & 0.33 & [114] \\
\hline Solvent-engineering & $\mathrm{FA}_{0.8} \mathrm{Cs}_{0.2} \mathrm{SnI}_{3}$ & $\begin{array}{l}\text { PEDOT:PSS/perovskite/ } \\
\text { PCBM/Bis-C } 60 / \mathrm{Ag}\end{array}$ & 0.24 & 16.05 & 35.8 & 1.38 & [114] \\
\hline
\end{tabular}


Table 1 (continued)

\begin{tabular}{|c|c|c|c|c|c|c|c|}
\hline Preparation process & Absorber & Device architecture & $V_{\mathrm{oc}}(\mathrm{V})$ & $J_{\mathrm{sc}}\left(\mathrm{mA} \mathrm{cm}^{-2}\right)$ & $\mathrm{FF}(\%)$ & PCE $(\%)$ & References \\
\hline Solvent-engineering & $\mathrm{FASnI}_{3}$ & $\begin{array}{l}\text { PEDOT:PSS/perovskite/ } \\
\text { PCBM/Bis-C } 60 / \mathrm{Ag}\end{array}$ & 0.04 & 11.73 & 23.4 & 0.11 & {$[114]$} \\
\hline Solvent-engineering & $\mathrm{PEA}_{2} \mathrm{SnI}_{4}$ & $\begin{array}{l}\mathrm{NiO}_{\mathrm{x}} / \text { perovskite/PCBM/BCP/ } \\
\mathrm{Ag}\end{array}$ & 0.61 & 22.0 & 70.1 & 9.41 & [123] \\
\hline Solvent-engineering & $\left(\mathrm{BA}_{0.5} \mathrm{PEA}_{0.5}\right)_{2} \mathrm{FA}_{3} \mathrm{Sn}_{4} \mathrm{I}_{13}$ & $\begin{array}{l}\text { PEDOT:PSS/perovskite/C } 60 / \\
\text { LiF/Al }\end{array}$ & 0.60 & 21.82 & 66.73 & 8.82 & {$[126]$} \\
\hline Solvent-engineering & $\mathrm{AVA}_{2} \mathrm{FA}_{\mathrm{n}-1} \mathrm{Sn}_{\mathrm{n}} \mathrm{I}_{3 \mathrm{n}+1}$ & $\begin{array}{l}\text { PEDOT:PSS/perovskite/ } \\
\text { PCBM/BCP/Ag }\end{array}$ & 0.61 & 21.0 & 68 & 8.71 & [128] \\
\hline Solvent-engineering & $(4 \mathrm{AMP})(\mathrm{FA})_{3} \mathrm{Sn}_{4} \mathrm{I}_{13}$ & c- $\mathrm{TiO}_{2} / \mathrm{ZrO}_{2} /$ perovskite $/ \mathrm{C}$ & 0.64 & 14.9 & 44.3 & 4.42 & [131] \\
\hline Quantum rods & $\mathrm{CsSnI}_{3}$ & $\begin{array}{l}{\mathrm{c}-\mathrm{TiO}_{2} / \text { perovskite/spiro-MeO- }}^{\mathrm{TAD} / \mathrm{Au}}\end{array}$ & 0.86 & 23.2 & 65 & 12.96 & [145] \\
\hline Quantum rods & $\mathrm{CsSnBr}_{3}$ & $\begin{array}{l}\text { c- } \mathrm{TiO}_{2} / \text { perovskite/spiro-MeO- } \\
\mathrm{TAD} / \mathrm{Au}\end{array}$ & 0.85 & 21.23 & 58 & 10.46 & {$[145]$} \\
\hline Quantum rods & $\mathrm{CsSnCl}_{3}$ & $\begin{array}{l}\mathrm{c}-\mathrm{TiO}_{2} / \text { perovskite/spiro-MeO- } \\
\mathrm{TAD} / \mathrm{Au}\end{array}$ & 0.87 & 19.82 & 56 & 9.66 & {$[145]$} \\
\hline Sequential deposition & $\mathrm{FASnI}_{3}+10 \% \mathrm{SnF}_{2}+\mathrm{TMA}$ & $\begin{array}{l}\mathrm{SnO}_{2} / \mathrm{C}_{60} / \text { perovskite/spiro- } \\
\text { MeOTAD/Ag }\end{array}$ & 0.31 & 21.65 & 64.7 & 4.34 & {$[83]$} \\
\hline Sequential deposition & $\mathrm{FASnI}_{3}+10 \% \mathrm{SnF}_{2}+\mathrm{TMA}$ & 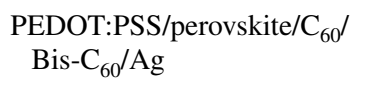 & 0.47 & 22.45 & 0.68 & 7.09 & {$[83]$} \\
\hline Sequential deposition & $\mathrm{FASnI}_{3}$ & $\begin{array}{l}\text { PEDOT:PSS/perovskite/C } 60 \\
\text { BCP/Ag }\end{array}$ & 0.33 & 17.78 & 67.9 & 3.98 & [146] \\
\hline Thermal evaporation & $\mathrm{MASnBr}_{3}$ & c-TiO $2 /$ perovskite/P3HT/Au & 0.498 & 4.27 & 49.1 & 1.12 & [77] \\
\hline Thermal evaporation & $\mathrm{CsSnBr}_{3}+2.5 \% \mathrm{SnF}_{2}$ & $\mathrm{MoO}_{3} /$ perovskite $/ \mathrm{C}_{60} / \mathrm{BCP} / \mathrm{Ag}$ & 0.40 & 2.4 & 55 & 0.55 & [147] \\
\hline Thermal evaporation & $(\mathrm{PEA}, \mathrm{FA}) \mathrm{SnI}_{3}$ & $\begin{array}{l}\text { LiF/PEDOT:PSS/perovskite/ } \\
\mathrm{C}_{60} / \mathrm{BCP} / \mathrm{Ag}\end{array}$ & 0.47 & 20.07 & 74 & 6.98 & [148] \\
\hline Thermal evaporation & $\mathrm{MASnI}_{3}$ & $\begin{array}{l}\text { PEDOT:PSS/TPD/perovskite/ } \\
\mathrm{C}_{60} / \mathrm{BCP} / \mathrm{Ag}\end{array}$ & 0.377 & 12.1 & 36.6 & 1.7 & [149] \\
\hline Thermal evaporation & $\mathrm{CsSnI}_{3}$ & ITO/perovskite/Au/Ti & 0.42 & 4.80 & 22 & 0.88 & [87] \\
\hline Direct dropping & $\mathrm{MASnIBr}_{1.8} \mathrm{Cl}_{0.2}+20 \% \mathrm{SnF}_{2}$ & $\begin{array}{l}\mathrm{c}-\mathrm{TiO}_{2} / \mathrm{m}-\mathrm{TiO}_{2} / \mathrm{m}-\mathrm{Al}_{2} \mathrm{O}_{3} / \\
\text { perovskite/C }\end{array}$ & 0.38 & 13.99 & 57.3 & 3.11 & {$[150]$} \\
\hline Hot-dropping & $\mathrm{CsSnIBr}_{2}+60 \% \mathrm{SnF}_{2}+\mathrm{H}_{3} \mathrm{PO}_{2}$ & $\begin{array}{l}\mathrm{c}-\mathrm{TiO}_{2} / \mathrm{m}-\mathrm{TiO}_{2} / \mathrm{m}-\mathrm{Al}_{2} \mathrm{O}_{3} / \\
\text { perovskite } / \mathrm{C}\end{array}$ & 0.31 & 17.4 & 56 & 3.2 & [151] \\
\hline Solvent-engineering & $\mathrm{FASnI}_{3}+\mathrm{PEABr}+10 \% \mathrm{SnF}_{2}$ & $\begin{array}{l}\text { PEDOT:PSS/perovskite/C }{ }_{60} / \\
\mathrm{BCP} / \mathrm{Cu}\end{array}$ & 0.45 & 24.87 & 63 & 7.05 & {$[152]$} \\
\hline Solvent-engineering & $\begin{array}{l}\mathrm{FASnI}_{3}+2.5 \% \mathrm{~N}_{2} \mathrm{H}_{5} \mathrm{Cl}+10 \% \\
\mathrm{SnF}_{2}\end{array}$ & $\begin{array}{l}\text { PEDOT:PSS/perovskite/ } \\
\text { PCBM/BCP/Ag }\end{array}$ & 0.455 & 17.64 & 67.3 & 5.40 & [153] \\
\hline Solvent-engineering & $\mathrm{FASnI}_{3}+12 \% \mathrm{SnF}_{2}$ & $\begin{array}{l}\text { PEDOT:PSS(PEG)/perovskite/ } \\
\text { PCBM/BCP/Ag }\end{array}$ & 0.455 & 17.64 & 67.3 & 5.40 & {$[153]$} \\
\hline Solvent-engineering & $\mathrm{MASnI}_{3}+20 \% \mathrm{SnF}_{2}$ & $\begin{array}{l}\text { PEDOT:PSS/perovskite/C }{ }_{60} / \\
\text { BCP/Ag }\end{array}$ & 0.45 & 11.82 & 40 & 2.14 & [154] \\
\hline Solvent-engineering & $\mathrm{FASnI}_{3}+1 \% \mathrm{EDAI}_{2}$ & $\begin{array}{l}\text { PEDOT:PSS/perovskite/C } 60 \\
\text { BCP/Ag }\end{array}$ & 0.583 & 21.3 & 0.72 & 8.9 & [155] \\
\hline Solvent-engineering & $\mathrm{FASnI}_{3}$ & $\begin{array}{l}\text { PEDOT:PSS/perovskite/C } 60 \\
\text { BCP/Ag }\end{array}$ & 0.638 & 21.95 & 0.725 & 10.16 & {$[156]$} \\
\hline
\end{tabular}

quality and reduced defect density, and the final device showed a PCE of 7.3\% [102]. More importantly, about 50\% of their original PCE was maintained after the $500 \mathrm{~h}$ storage in air. Soon after, Han et al. employed a $\pi$-conjugated Lewis base molecule 2-cyano-3-[5-[4-(diphenylamino) phenyl]-2-thienyl]-propenoic acid (CDTA) with high electron density to systematically control the crystallization rate of $\mathrm{FASnI}_{3}$ perovskite [103]. They obtained a compact and uniform perovskite film with greatly increased carrier lifetime via forming stable intermediate phase with 
the Sn-I frameworks. Meanwhile, the introduction of the $\pi$-conjugated system also retarded the permeation of moisture into perovskite crystal, which significantly suppressed the film degradation in air [103]. The perovskite solar cell prepared on this basis achieved a PCE of $10.1 \%$ and maintained over $90 \%$ of its initial value after $1000 \mathrm{~h}$ light soaking in air. Another work is Han et al. introduced a liquid formic acid as a reducing solvent in the $\mathrm{FASnI}_{3}$ perovskite precursor solution to product the $\mathrm{FASnI}_{3}$ perovskite film with high crystallinity, low $\mathrm{Sn}^{4+}$ content, reduced background doping, and low electronic trap density. As a result, the lead-free tin halide PSC achieved $10.37 \%$ efficiency [104].

In addition to additives, the use of antisolvents also greatly promoted the film quality of $\mathrm{FASnI}_{3}$ perovskites. Typical antisolvents include chlorobenzene (CB) [105], toluene (TL) [106], diethyl ether (DE) [107], and other aprotic nonpolar solvents. Here, it is noteworthy that the antisolvents should be miscible with DMSO solvent, but insoluble for perovskites. Liao et al. used such a solvent-engineering method to fabricate $\mathrm{FASnI}_{3}$ perovskites and tuned the film morphologies with different antisolvents (Fig. 3e-h) [96]. They obtained high-quality, uniform and fully covered $\mathrm{FASnI}_{3}$ perovskite thin films. Among these antisolvents, dripping with diethyl ether onto $\mathrm{FASnI}_{3}$ produced the best $\mathrm{FASnI}_{3}$ thin films with high uniformity and full coverage. With improved film quality and proper structure design, the champion $\mathrm{FASnI}_{3}$ device achieved a PCE of $6.22 \%$ with $V_{\text {oc }}$ of $0.465 \mathrm{~V}, J_{\mathrm{sc}}$ of $22.07 \mathrm{~mA} \mathrm{~cm}^{-2}$, and FF of $60.67 \%$. Furthermore, such device exhibited a weak $J-V$ hysteresis behavior [96].

Recently, a new type of "hollow" perovskites has been prepared. Chen et al. reported a successful incorporation of medium size cations ethylenediammonium (en) into the $3 \mathrm{D} \mathrm{FASnI}_{3}$ perovskite structure and they denoted it as $\{\mathrm{en}\}$ FASnI $_{3}$ [91, 108]. Typically, change of A-site cation only results in a small bandgap alteration due to structural distortion, but for $\{$ en $\} \mathrm{FASnI}_{3}$, its bandgap can be tuned in a wide range of 1.3-1.9 eV via simply increasing the en amount [108]. Actually, adding en could improve film morphology, reduce background carrier density and increase carrier lifetime, all of which contribute to the resultant PCE and stability of Sn-based solar cells [70]. Based on this viewpoint, the $\{$ en $\} \mathrm{FASnI}_{3}$ device achieved a $7.14 \%$ PCE with higher $V_{\text {oc }}$ and FF. Similar results were also observed in $\mathrm{MASnI}_{3}$ and $\mathrm{CsSnI}_{3}$ solar cells [108]. Recently, Kanatzidis et al. also reported two other diammonium cations of propylenediammonium (PN) and trimethylenediammonium (TN) forming new hollow perovskites of $\{\mathrm{TN}\} \mathrm{FASnI}_{3}$ and $\{$ PN $\}$ FASnI $_{3}$ [109]. TN and PN with slightly larger size than en also improved device performances. The $\mathrm{FASnI}_{3}$ absorbers mixed with $10 \% \mathrm{PN}$ and $10 \%$ TN achieved enhanced PCEs of $5.85 \%$ and $5.53 \%$, respectively [109].

\section{(4) Mixed A Cations Sn-Based Perovskites}

Metal halide perovskites with mixed cations have been widely used in $\mathrm{Pb}$-based PSCs, part of which achieved record efficiencies and good stability due to cation mixturetriggered film morphology improvement and water and oxygen resistance increase as well as inhibition of carrier recombination within devices [20, 22, 110-113]. In this case, it is also a powerful strategy to develop highly efficient, stable Sn-based PSCs with mixed A cations perovskites. Liu et al. first synthesized Sn-based perovskites with mixed cations of MA and Cs and applied $\mathrm{MA}_{0.9} \mathrm{Cs}_{0.1} \mathrm{SnI}_{3}$ to PSCs [114]. The PCE was low $(0.33 \%)$ since the device was fabricated without any optimization. Later, Zhao et al. reported 3D Sn-based perovskites with mixed cations of MA and FA (Fig. 6d). Notably, the optical property exhibited an obvious alteration with the ratio of mixed cations (Fig. 6a-c, e-f). Among them, $(\mathrm{FA})_{0.75}(\mathrm{MA})_{0.25} \mathrm{SnI}_{3}$ perovskite solar cell with $10 \mathrm{~mol} \% \mathrm{SnF}_{2}$ generated a maximum PCE of $8.12 \%$ and an average value of $7.48 \% \pm 0.52 \%$ [88].

3.1.1.2 Low-Dimensional Sn-Based Perovskite As an alternative, reduced-dimensional (quasi-2D) perovskites have been widely investigated recently since they have showed superior enduring stability outperforming their 3D counterparts [115-117]. The existence of insulating organic spacer cations, e.g., PN [89], 2-hydroxyethylammonium (HEA) [118], guanidinium (GA) [119], butylammonium (BA) [120], polyethylenimine cation [121], cyclopropylammonium [122], and phenylethylammonium (PEA) on quasi2D perovskites, restrains self-doping effect and suppresses ion migration $[115,121]$, thus contributing to ameliorated moisture, oxygen, and thermal stability. However, the emergence of these insulating long-chain organic cations also leads to the anisotropy characteristics of the crystal, which significantly reduces device performance. That means the resulting quasi-2D PSCs with enhanced stability are at the expense of declining performance due to blocked charge transportation of these insulating organic spacers along vertical direction $[95,120,123]$. The highly vertically oriented perovskite films are considered as a prerequisite to address 
the low-efficiency issue in quasi-2D PSCs since in this situation these inorganic slabs are aligned in perpendicular to the substrates $[124,125]$. Various methods of forming highly vertically oriented films have been developed in Pb-based quasi-2D perovskites, but unfortunately, most of which are unsuitable for Sn-based counterparts due to different properties between $\mathrm{Pb}$ - and Sn-based perovskites [123, 124]. In the published cases of Sn-based PSCs, Kanatzidis et al. reported 2D Ruddlesden-Popper (RP) perovskites $\left(\mathrm{CH}_{3}\left(\mathrm{CH}_{2}\right)_{3} \mathrm{NH}_{3}\right)_{2}\left(\mathrm{CH}_{3} \mathrm{NH}_{3}\right)_{\mathrm{n}-1} \mathrm{Sn}_{\mathrm{n}} \mathrm{I}_{3 \mathrm{n}+1}$ with perpendicular orientation by using $\mathrm{N}, \mathrm{N}$-dimethylformamide as solvent [120]. For $n=4$, perpendicularly oriented 2D perovskites were fabricated whether the substrates were heated or not, while for $n=3$, only hot substrates of 120 centigrade were able to intensify perpendicular growth of 2D films. Huang et al. introduced mixed $n$-butylamine and PEA organic cations into 2D RP Sn perovskite to control the crystallization process and formed highly vertically oriented [(BA $\left.{ }_{0.5} \mathrm{PEA}_{0.5}\right)_{2} \mathrm{FA}_{3} \mathrm{Sn}_{4} \mathrm{I}_{13}$ ] 2D RP perovskites [126]. Benefitting from it, the PCE of the 2D Sn-based PSC was improved to $8.82 \%$. Liao et al. reported low-dimensional Sn-based perovskites by using FA and PEA mixed cations (Fig. 7a), and they adjusted the orientation of the perovskite domains by altering the PEA/FA ratios [127]. A highly oriented per- ovskite film perpendicular to the substrate was realized by adding 20\% PEA. As a result, the $\mathrm{FASnI}_{3}$ perovskite solar cells with $20 \%$ PEA achieved a maximum PCE of 5.94\% with enhanced stability [127]. Recently, Yuan et al. used the bifunctional cation 5-ammoniumvaleric acid (5-AVA) as the spacer to fabricate $\mathrm{AVA}_{2} \mathrm{FA}_{n-1} \mathrm{Sn}_{n} \mathrm{I}_{3 n+1}(\langle n\rangle=5)$ quasi-2D Sn-based perovskite, and by introducing appropriate amount of ammonium chloride $\left(\mathrm{NH}_{4} \mathrm{Cl}\right)$ additive, they obtained highly vertically oriented quasi-2D perovskite films which eventually enhanced the transportation of charge carriers between electrodes [128]. Actually, the $\mathrm{Cl}$ doping can also improve the electrical conductivity of perovskite film, as demonstrated by Li et al. [129].

In recent work, Padture et al. first reported the synthesis and photovoltaic performance of low-dimensional Dion-Jacobson Sn(II)-based halide perovskites (4AMP) $(\mathrm{FA})_{\mathrm{n}-1} \mathrm{Sn}_{\mathrm{n}} \mathrm{I}_{3 \mathrm{n}+1}$, but the PSC based on this perovskite only achieved an efficiency of $2.15 \%$ [130]. Compared with the Sn-based perovskite of the RP layered with a van der Waals gap between the adjacent unit cells, the unique structure of DJ layered Sn-based perovskites allows the inorganic stacks to be more uniform and closer, rendering better alignment and less displacement of perovskites [131]. Moreover, the
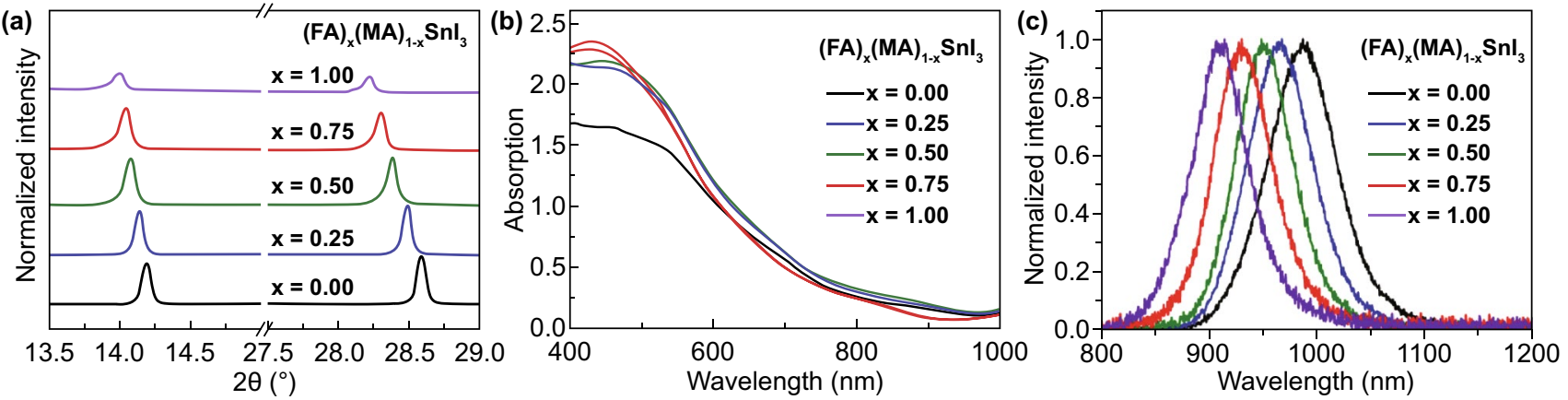

(d)
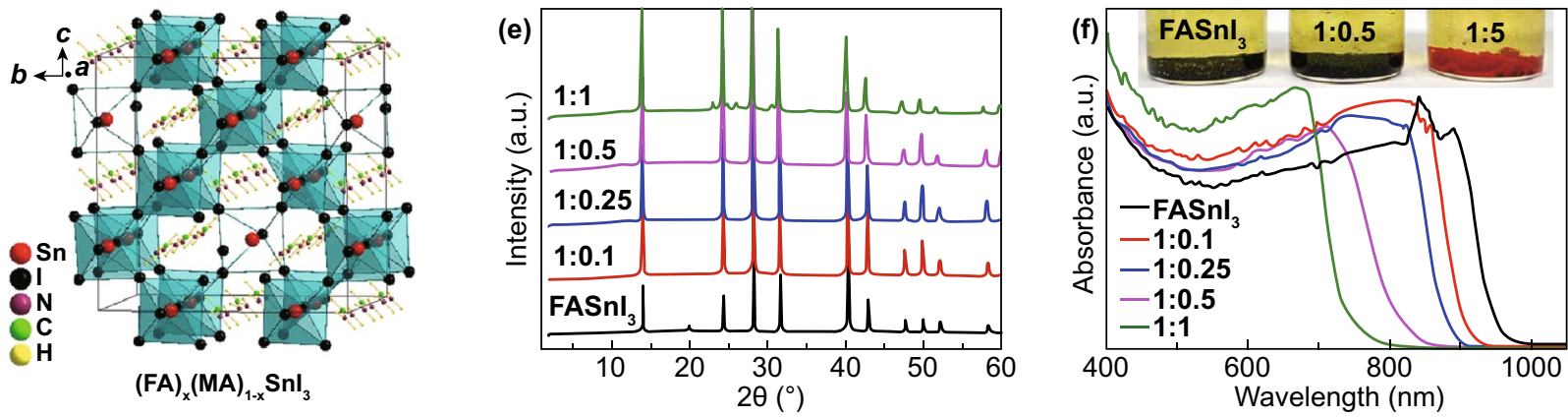

Fig. 6 a XRD patterns of one-step deposited (FA) $)_{x}(\mathrm{MA})_{1-\mathrm{x}} \mathrm{SnI}_{3}(\mathrm{x}=0.00,0.25,0.50,0.75$, and 1.00) films on ITO/poly(3,4ethylenedioxythiophene)-poly(styrenesulfonate) (PEDOT:PSS) substrates. b Absorption spectra and c normalized PL spectra of the different perovskite films on quartz substrates. d A $2 \times 2 \times 2$ supercell of $(\mathrm{FA})_{2} \mathrm{Sn}_{2} \mathrm{I}_{6}$ depicting a model of the hollow perovskite with two $\mathrm{SnI}_{2}$ vacancies $\left[(\mathrm{FA})_{16} \mathrm{Sn}_{14} \mathrm{I}_{44}\right]$. e XRD patterns and $\mathbf{f}$ optical absorption of the $\{\mathrm{en}\} \mathrm{FASnI}{ }_{3}$ perovskite crystals with various molar ratios of FA to \{en\}. Reproduced with permission from Ref. [88] 
shortened interlayer distance might reduce the barrier of charge transfer, which will benefit the charge transport of derived quasi-2D perovskite devices. It is particularly worth mentioning that the growth of the DJ phase perovskite layer can be controlled by selecting different diammonium organic cations as the intermediate spacer layer so that the growth direction is perpendicular to the substrate, which facilitates the transfer and collection of charge through the electrode $[124,132]$. These characteristics will make DJ Sn-based perovskites to be a promising class of materials for photovoltaic applications.

Kanatzidis et al. reported some hexagonal polytypes and low-dimensional structures of hybrid Sn iodide perovskites, which employed various small cations such as trimethylammonium $\left(\mathrm{TMA}^{+}\right)$, imidazolium $\left(\mathrm{IM}^{+}\right)$, guanidinium $\left(\mathrm{GA}^{+}\right)$, ethylammonium $\left(\mathrm{EA}^{+}\right)$, acetamidinium $\left(\mathrm{ACA}^{+}\right)$, and isopropylamine $\left(\right.$ IPA $\left.^{+}\right)$(Fig. 7b-e) [133]. Due to the moderate size of these cations, a variety of new $\mathrm{ASnX}_{3}$ polytypes were formed by combinations of corner-sharing octahedra (perovskite) and face-sharing octahedra (perovskitoid), which have the potential for lead-free solar cells [134]. Finally, we summarize the current research results of Sn-based perovskite solar cells in Table 1 [70, 71, 80-83, 87-89, 96, 97, 101-104, 118-120, 124, 133-156].

To further improve the efficiency of tin-based perovskite, device structure manipulation was also conducted by Ning et al., who used indene- $\mathrm{C}_{60}$ bisadduct as the ETL to fabricate solar cells based on $\mathrm{PEA}_{\mathrm{x}} \mathrm{FA}_{1-\mathrm{x}} \mathrm{SnI}_{3}$ perovskite, yielding a high efficiency of $12.4 \%$ [135]. In addition to efficiency, the stability issue of tin-based PSCs also restrained their applications. Mathews et al. encapsulated $\mathrm{FASnI}_{3}$ PSCs and realized more stable PSCs with over $80 \%$ of its initial PCE being maintained after 1-month storage in a $\mathrm{N}_{2}$ environment, which is much better than the unencapsulated counterparts that fully decomposed within two weeks [71]. In addition, Kanatzidis et al. studied the encapsulated 2D $(\mathrm{BA})_{2}(\mathrm{MA})_{3} \mathrm{Sn}_{4} \mathrm{I}_{13}$ PSCs and their test results showed that the encapsulated device retained more than $90 \%$ of its initial performance after 1 month and dropped only to $\sim 50 \%$ after 4 months in a glove box full of $\mathrm{N}_{2}$ [120]. This encouraging stability is closely related to the intrinsically stable $2 \mathrm{D}$ perovskite structure as well as the encapsulation that sufficiently blocked the invasion of water and oxygen. Other researchers have also proposed in their respective work that it is possible to package Sn-based solar cells to improve stability [71, 103, 104, 120, 136, 155, 156].
In summary, there is still a large room for efficiency and stability enhancements of Sn-based perovskites. Future works should focus on optimization of perovskite composition and selection of effective additives to fabricate stable and high-quality perovskite film with less $\mathrm{Sn}^{2+}$ oxidation. Besides, reasonable choice of hole/electron transport materials is very critical for enhancing device performance and stability of the Sn-based PSCs. Protecting of solar cells with industrial encapsulation techniques will also be a viable approach to improve the long-term stability of the Sn-based PSCs and push it to the commercial market.

\subsubsection{Ge-based Perovskite}

Previous reports have shown that metal ions owning external $n s^{2}$ electronic structure with low ionization energy can enhance light absorption efficiency and carrier diffusion length of $\mathrm{ABX}_{3}$ structure. $\mathrm{Ge}^{2+}$ has similar outer $\mathrm{ns}^{2}$ electronic structure $\left(4 \mathrm{~s}^{2}\right)$ to $\mathrm{Sn}^{2+}\left(5 \mathrm{~s}^{2}\right)$ and $\mathrm{Pb}^{2+}\left(6 \mathrm{~s}^{2}\right)$, but smaller ionic radii than $\mathrm{Sn}^{2+}$ and $\mathrm{Pb}^{2+}$ [64]. Ge-based perovskites containing $\mathrm{MAGeI}_{3}, \mathrm{CsGeI}_{3}$ and $\mathrm{FAGeI}_{3}$ have also been demonstrated to be stable at temperature of up to $150{ }^{\circ} \mathrm{C}$. According to these data, Mathews et al. considered that $\mathrm{Ge}$ could replace $\mathrm{Pb}$ as a new type of lead-free perovskite material [64]. Actually, the bandgaps of the Ge-based perovskites can be adjusted close to the conventional lead halide perovskite of $1.5 \mathrm{eV}$ via altering A-site cations.

Kanatzidis et al. synthesized a series of $\mathrm{AGeI}_{3}$ perovskite compounds and characterized their structural, electronic and optical properties in 2015 [61]. The bandgaps of the Ge-based perovskites increase with the increased radius of the A cation. For instance, $\mathrm{CsGeI}_{3}$ has an optical bandgap of $1.63 \mathrm{eV}$, whereas other $\mathrm{AGeI}_{3}$ perovskites universally exhibit wide bandgap values of $\geq 2.0 \mathrm{eV}$. These $\mathrm{AGeI}_{3}$ perovskites are rhombohedral crystal structures at room temperature [60]. Kanatzidis et al. [61] used the pyramidal $\left[\mathrm{GeI}_{3}\right]^{-}$building block to synthesize a series of Ge-based perovskites and adjusted their A-site cations. The experimental results showed that $\mathrm{CsGe}_{3}, \mathrm{MAGeI}_{3}$,

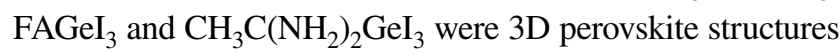
with direct bandgaps of 1.6, 1.9, 2.2, and $2.5 \mathrm{eV}$, respectively, while $\mathrm{C}\left(\mathrm{NH}_{2}\right)_{3} \mathrm{GeI}_{3},\left(\mathrm{CH}_{3}\right)_{2} \mathrm{C}(\mathrm{H}) \mathrm{NH}_{3} \mathrm{GeI}_{3}$, and $\left(\mathrm{CH}_{3}\right)_{3} \mathrm{NHGeI}_{3}$ were 1D infinite chain structures separately with indirect bandgaps of 2.7, 2.5, and $2.8 \mathrm{eV}$, among which $\mathrm{CsGeI}_{3}$ exhibited the highest optical absorption coefficient (Fig. 8a) and showed much more application potential in photovoltaic field. 

(a) $\begin{aligned} & \mathrm{FA}^{+} \\ & \mathrm{Sn}^{2+}+\mathrm{I}^{-} \\ & \mathrm{PEA}^{+}\end{aligned}$

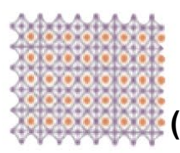

$(101)$

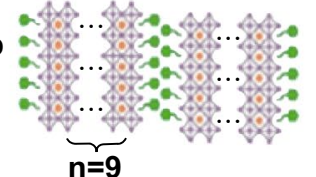

$20 \%$

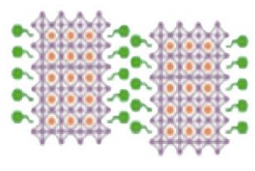

$40 \%$

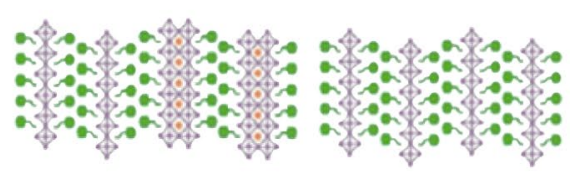

$80 \%$
$100 \%$
3D

(b)
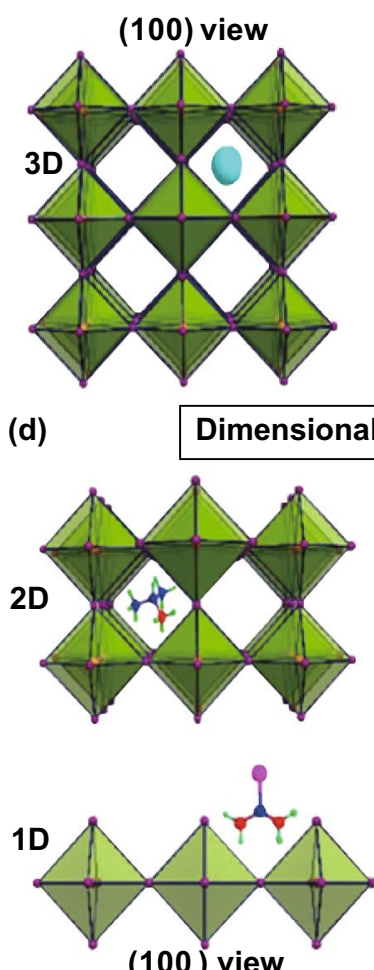

(100) view
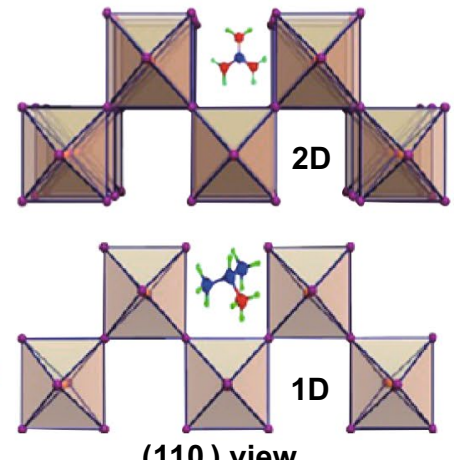

Corner-Sharing Perovskite (110) view

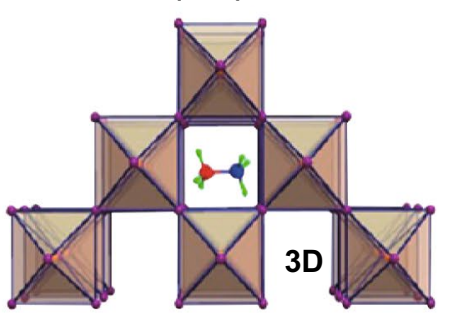

Reduced Perovskites

(110) view

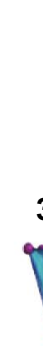

(c)
2D

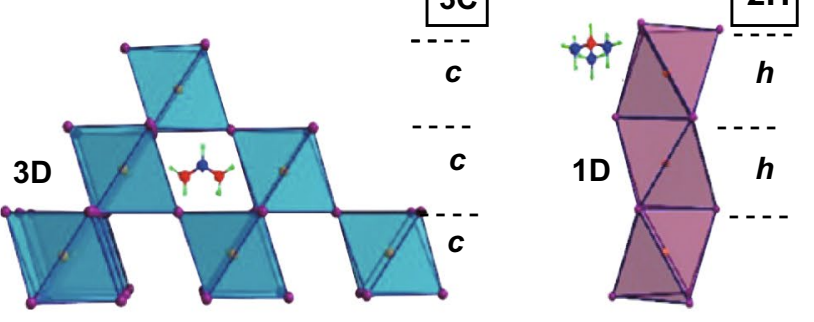

(e)

\section{Hexagonal Perovskite Polytypes}

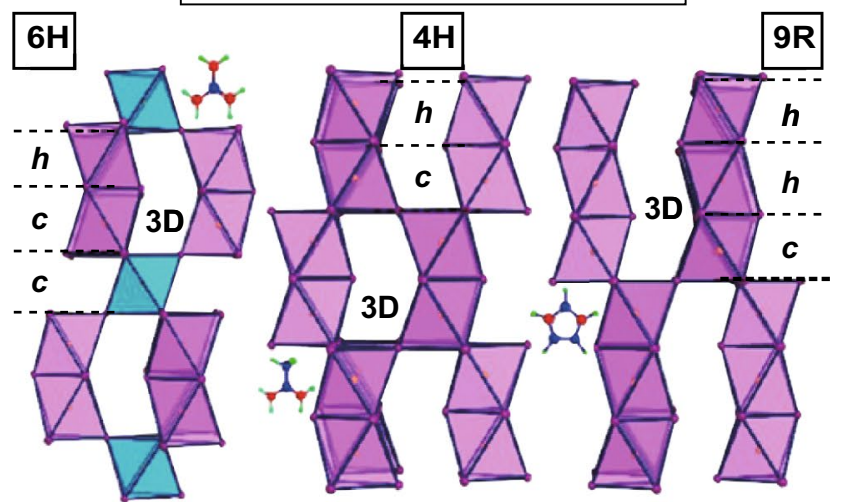

Fig. 7 a Schematic structures of mixed FA/PEA Sn perovskites with PEAI/FAI doping ratios of 0, 20, 40, 60, 80, and 100\%. Reproduced with permission from Ref. [127]. b Three alternative views of the archetypal 3D perovskite structure viewed along the (100), (110), and (111) cleavage planes. c "Perovskitoid" face-sharing building block of the 1D structures obtained for $\mathrm{t}>1$ representing the archetypal hexagonal polytype. d Perovskite structures obtained through dimensional reduction featuring corner-sharing 2D sheets and 1D chains through the (100) and (110) cleavage planes, respectively. e Hexagonal perovskite polytypes obtained from a linear combination of the corner-sharing 3D perovskite along the (111) cleavage plane and the 1D face-sharing polytype. The " $\mathrm{h}$ " and "c" symbols indicate hexagonal and cubic layers, respectively, and serve in identifying the layer sequence that characterizes the polytype. Reproduced with permission from Ref. [135]

The photoluminescence (PL) measurements of $\mathrm{CsGeI}_{3}$ single crystals exhibited two peaks at $0.82 \mu \mathrm{m}(1.51 \mathrm{eV})$ and $1.15 \mu \mathrm{m}(1.08 \mathrm{eV})$, which were respectively assigned to the interband transition at $\alpha . \mathrm{k}=(\pi / \mathrm{a})(111)$ and the energy band change (Fig. 9a). The energy bands of the Ge-based perovskites with different A cations are shown in Fig. 9b. Fourier transform infrared spectroscopy showed that the transparent range of $\mathrm{CsGeI}_{3}$ can be extended from $\sim 2$ to $>12 \mu \mathrm{m}$. The short-wave cutoff is mainly limited by the energy band, and the longest infrared transparency wavelength may originate from the phonon absorption of crystal lattice, and a specific rhombohedral crystal structure can be generated at room temperature [157]. Mathews et al. used hypophosphorous acid to improve the solubility of Ge perovskite precursor in organic solvent and prepared Ge-based perovskite films [60]. According to the SEM images (Fig. 9d-f), the films of $\mathrm{MAGeI}_{3}$ and $\mathrm{CsGeI}_{3}$ exhibited nearly full-coverage morphology, but the $\mathrm{FAGeI}_{3}$ film showed a poor quality. Based 
on these films, they then fabricated devices on mesoporous $\mathrm{TiO}_{2}$ structures. MAGeI ${ }_{3}$ and $\mathrm{CsGeI}_{3}$ solar cells showed $J_{\mathrm{sc}} \mathrm{s}$ of 4.0 and $5.7 \mathrm{~mA} \mathrm{~cm}^{-2}$ and PCEs of 0.11 and $0.2 \%$, respectively, as shown in Fig. 9c. In contrast, $\mathrm{FAGeI}_{3}$ solar cell with poor film morphology displayed no photocurrent.

Kopacic et al. [158] demonstrated that chemical composition engineering of $\mathrm{MAGeI}_{3}$ significantly improved performances of solar cells and simultaneously enhanced their stability. By introducing bromide anion to partially replace idiode, the PCE based on $\mathrm{MAGeI}_{2.7} \mathrm{Br}_{0.3}$ was increased up to $0.57 \%$.

Generally, Ge-based perovskites exhibit obviously different energy structures from the $\mathrm{Pb}$-based ones and thus suitable ETL and HTL should be optimized to improve charge extraction efficiency in the device. More importantly, stabilization of $\mathrm{Ge}^{2+}$ in Ge-based perovskite is also a pretty challenging task to be solved. Otherwise, the stability issue and extremely low PCE will limit broader photovoltaic applications of this kind of perovskites.

\subsubsection{Transition Metal Halide Perovskite}

Divalent transition metal cations (e.g., $\mathrm{Cu}^{2+}, \mathrm{Fe}^{2+}, \mathrm{Zn}^{2+}$ ) have also been considered as the candidates of $\mathrm{Pb}$-free perovskites, which can be synthesized via a variety of routes to tune photovoltaic properties [63, 159]. Because of small ionic radii for transition metal ions (e.g., $0.73 \mathrm{~nm}$ for $\mathrm{Cu}(\mathrm{II})$ and $0.78 \mathrm{~nm}$ for $\mathrm{Fe}(\mathrm{II})$ ) that far deviated from tolerance factor of 1 , 3D structure (e.g., $\mathrm{K}_{2} \mathrm{NiF}_{4}$ ) cannot be maintained and it turns into stable $2 \mathrm{D}$ layered structure along $<100>,<110>$ and $<111>$ orientations [160].

$\mathrm{Cu}^{2+}$ is a promising $\mathrm{Pb}$-free perovskite in consideration of its good stability, earth abundance, low-cost and sufficient absorption in near-infrared region. Currently, some $\mathrm{Cu}$-based perovskites have been developed for solar cell applications [63, 161, 162]. Cortecchia et al. first used the $2 \mathrm{D}$ crystal structure of $\mathrm{MA}_{2} \mathrm{CuCl}_{\mathrm{x}} \mathrm{Br}_{4-\mathrm{x}}$ (Fig. 10a) as a light absorbing layer to fabricate solar cell [63]. They mixed $\mathrm{MABr}, \mathrm{MACl}, \mathrm{CuCl}_{2}$ and $\mathrm{CuBr}_{2}$ in $\mathrm{DMSO}$ as the precursor
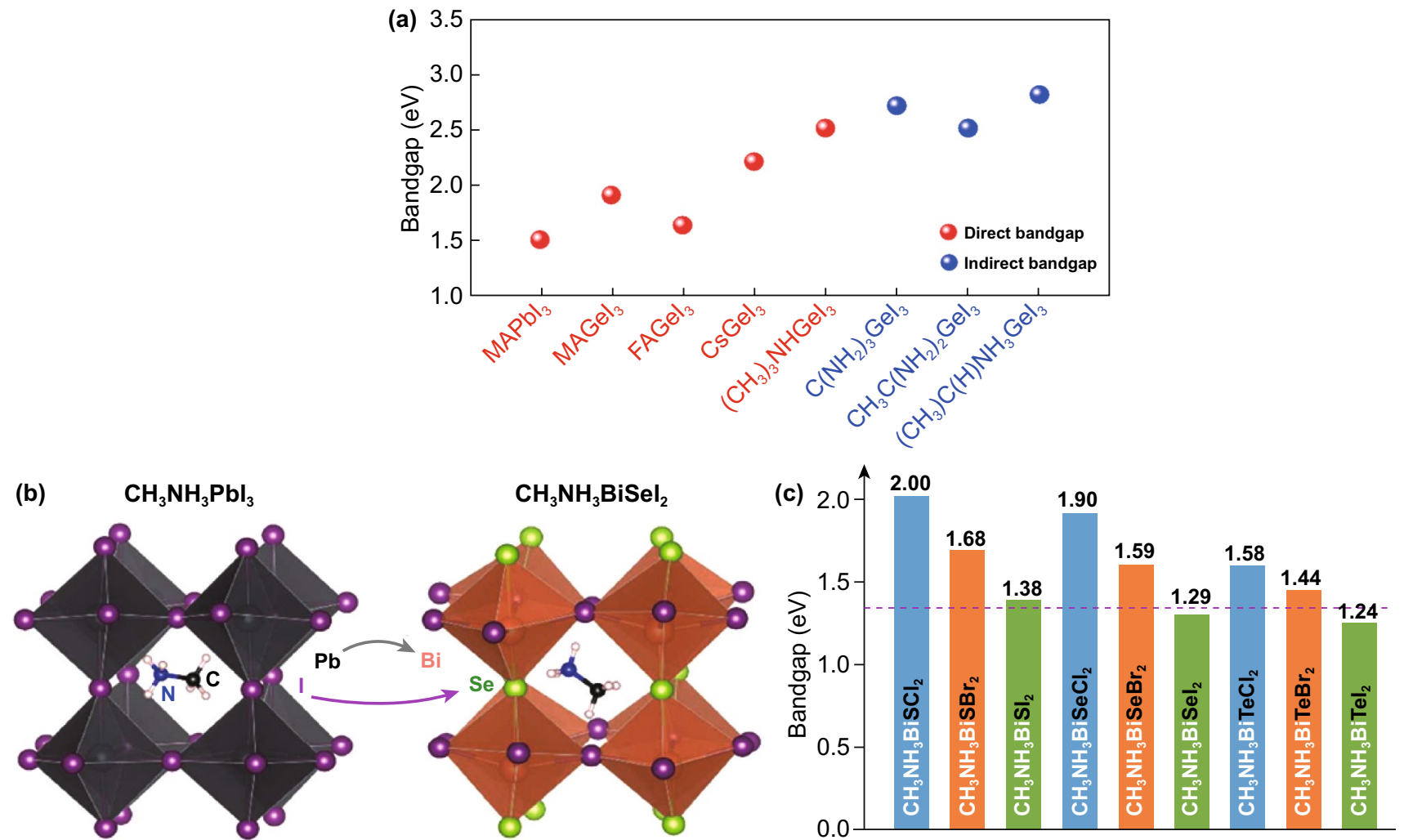

Fig. 8 a Bandgaps of various Ge-based perovskite materials compared with $\mathrm{MAPbI}_{3}$. b Atomic structures of $\mathrm{MAPbI}_{3}$ and $\mathrm{MABiSeI}_{2}$. c Calculated bandgaps of $\mathrm{CH}_{3} \mathrm{NH}_{3} \mathrm{BiXY}$ compounds (with $\mathrm{X}=\mathrm{S}$, Se, Te and $\mathrm{Y}=\mathrm{Cl}, \mathrm{Br}$, or I) using the Heyd-Scuseria-Ernzerhof functional with spinorbit coupling. The dashed line marks the optimal bandgap for single-junction solar cell according to the Shockley-Queisser theory. Reproduced with permission from Ref. [52] 
solution and tuned the bandgaps of $\mathrm{MA}_{2} \mathrm{CuCl}_{\mathrm{x}} \mathrm{Br}_{4-\mathrm{x}}$ from 2.48 to $1.80 \mathrm{eV}$ via changing the $\mathrm{Br}$ content (Fig. 10b). $\mathrm{Cu}-$ based perovskites exhibited a narrow bandgap with low conduction band compared to other non-transition metal perovskite compounds, whose conduction band is derived from the unoccupied $\mathrm{Cu} 3 \mathrm{~d}$ orbitals hybridized with $\mathrm{Br} /$ $\mathrm{Cl} \mathrm{p}$ orbitals. The $\mathrm{MA}_{2} \mathrm{CuCl}_{\mathrm{x}} \mathrm{Br}_{4-\mathrm{x}}$ layer was fabricated on the $\mathrm{FTO} /$ mesoporous $\mathrm{TiO}_{2}$ substrate by spin coating process. A PCE of $0.017 \%$ was obtained with extremely low $J_{\text {sc }}$ of $216 \mu \mathrm{A} \mathrm{cm}^{-2}$ and $V_{\text {oc }}$ of $0.256 \mathrm{~V}$ (Fig. 10c). In addition, they also prepared planar heterojunction cells based on $\mathrm{MA}_{2} \mathrm{CuCl}_{\mathrm{x}} \mathrm{Br}_{4-\mathrm{x}}$, but the PCE was far less than $0.017 \%$. The higher PCE for mesoporous structure solar cell arises from improved charge extraction and vertical charge transport along with the destruction of the $2 \mathrm{D}$ structure of $\mathrm{MA}_{2} \mathrm{CuCl}_{\mathrm{x}} \mathrm{Br}_{4-x}$ perovskite by mesoporous $\mathrm{TiO}_{2}$. The solar cells with $\mathrm{MA}_{2} \mathrm{CuCl}_{\mathrm{x}} \mathrm{Br}_{4-\mathrm{x}}$ as the light absorber showed better stability due to the essential role of $\mathrm{Cl}^{-}$, but unfortunately, the low absorption coefficient and heavymass hole limited their device performances. Subsequent works showed that $\left(\mathrm{C}_{6} \mathrm{H}_{5} \mathrm{CH}_{2} \mathrm{NH}_{3}\right)_{2} \mathrm{CuBr}_{4},\left(\rho-\mathrm{FC}_{6} \mathrm{H}_{5} \mathrm{C}_{2} \mathrm{H}_{4}\right.$ $\left.\mathrm{NH}_{3}\right)_{2} \mathrm{CuBr}_{4}$ and $\left(\mathrm{CH}_{3}\left(\mathrm{CH}_{2}\right)_{3} \mathrm{NH}_{3}\right)_{2} \mathrm{CuBr}_{4}$ based solar cells had slightly higher PCEs of $0.2 \%, 0.51 \%$, and $0.63 \%$ [161, 162].

On the whole, $\mathrm{Cu}$-based perovskites are relatively stable compared to other transition metal perovskite materials despite of the universially low conversion efficiency. How to reduce their wide optical bandgap and improve their charge transfer rate is the bottleneck problems limiting the further development of this type of perovskites. We believe that $\mathrm{Cu}$-based perovskites will be an important part of lead-free perovskite solar cells once overcoming these problems.

\subsection{Lead Replacement by Heterovalent Elements}

\subsection{1 $A_{3} B_{2} X_{9}$ Perovskite}

Bi-based perovskites represent a successful example in lead-free perovskite materials family with low toxicity, air stability, and a fair degree of tunability. Abulikemu et al. successfully fabricated $\left(\mathrm{CH}_{3} \mathrm{NH}_{3}\right)_{3} \mathrm{Bi}_{2} \mathrm{I}_{9}$ powders, millimeter-scale single crystals and thin films with solvent-based crystallization methods (Fig. 11a, b) [163]. Compared with Pb-based counterpart, $\left(\mathrm{CH}_{3} \mathrm{NH}_{3}\right)_{3} \mathrm{Bi}_{2} \mathrm{I}_{9}$ perovskite has better
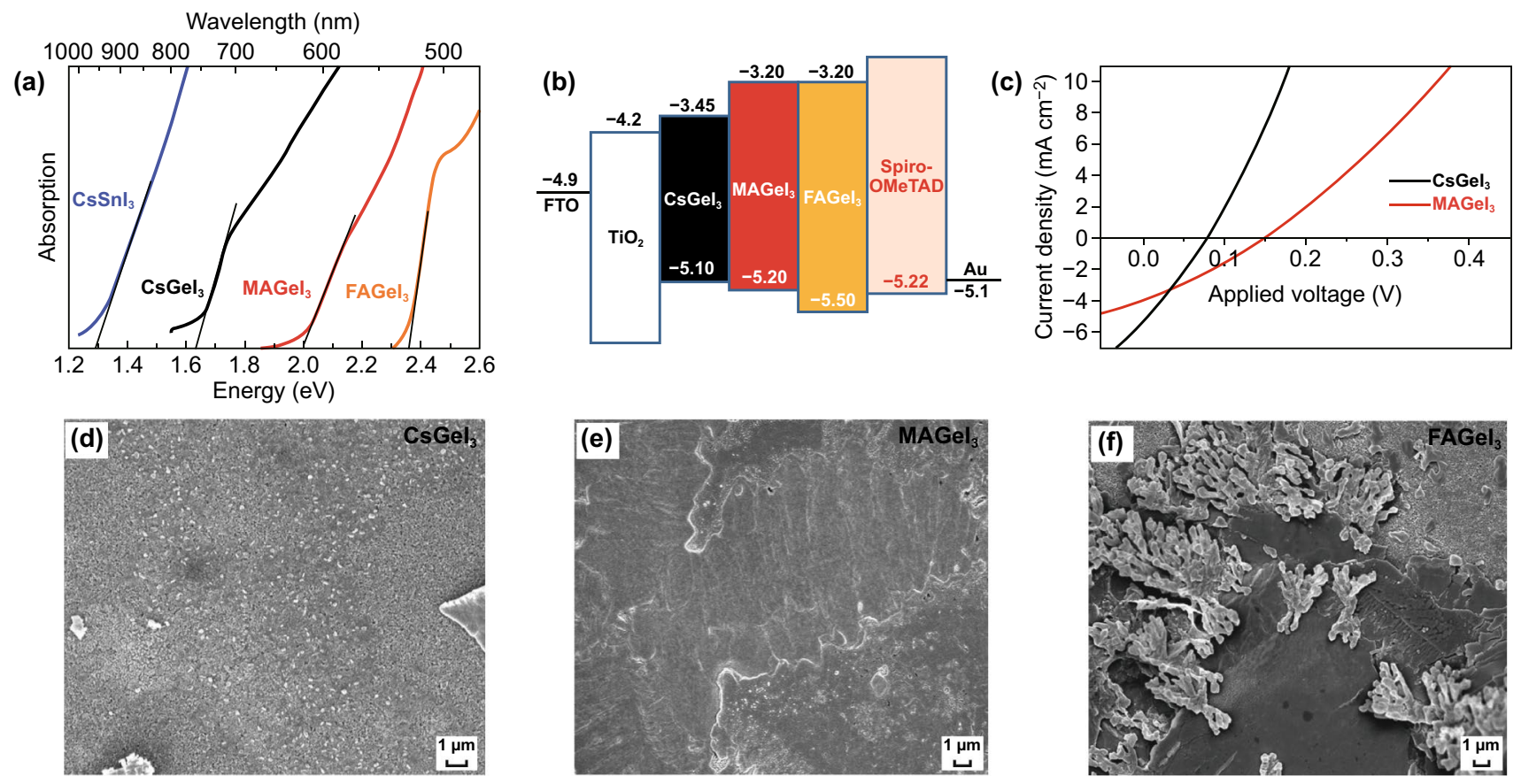

Fig. 9 a Tauc plot for $\mathrm{CsSnI}_{3}, \mathrm{CsGeI}_{3}, \mathrm{MAGeI}_{3}$, and $\mathrm{FAGeI}_{3}$ showing optical bandgaps of 1.29, 1.63, 2.0, and $2.35 \mathrm{eV}$, respectively. b Energy level pattern for the PSCs with $\mathrm{CsGeI}_{3}, \mathrm{MAGeI}_{3}$, and $\mathrm{FAGeI}_{3}$ absorbers. c Comparison of $J-V$ curves of $\mathrm{CsGeI}_{3}$ and $\mathrm{MAGeI}_{3}$ solar cells. d-f $\mathrm{SEM}$ images for $\mathrm{CsGeI}_{3}, \mathrm{MAGeI}_{3}$, and $\mathrm{FAGeI}_{3}$ films both deposited on the compact $\mathrm{TiO}_{2} /$ mesoporous $\mathrm{TiO}_{2}$ substrates. Reproduced with permission from Ref. [60] 
stability. In response to the rapid crystallization of perovskites during film formation, the researchers proposed two thin film annealing methods to improve the film-forming quality. One was an antisolvent assisted crystallization route, which dropped antisolvent of chlorobenzene during the spin coating process of precursors and then annealed at $70{ }^{\circ} \mathrm{C}$ for $10 \mathrm{~min}$ to form perovskite film. The other method was denominated saturated vapor crystallization. The perovskite film was fabricated without dropping chlorobenzene during the spin coating process, and then annealed in a closed Petri dish at $60{ }^{\circ} \mathrm{C}$ overnight to slow the solvent evaporation, which generated a highly oriented single-crystal film (Fig. 11b, c). Solar cell devices were fabricated based on these thin films, achieving an efficiency of $0.11 \%$, an FF of $31.80 \%$, an average $J_{\text {sc }}$ of $491.89 \mu \mathrm{A} \mathrm{cm}^{-2}$ and a $V_{\text {oc }}$ of $0.7216 \mathrm{~V}$ (Fig. 11d, e). However, the external quantum efficiency (EQE) spectrum demonstrated that the device produced photocurrent merely at a short wavelength, with most part of the visible spectrum missed (e.g., above $500 \mathrm{~nm}$ ) [163]. The explanation given by the researchers was that the depletion of EQE in the $\left(\mathrm{CH}_{3} \mathrm{NH}_{3}\right)_{3} \mathrm{Bi}_{2} \mathrm{I}_{9}$ film was attributed to its inhomogeneity and discontinuity between crystallites in terms of high recombination of photogenerated charges, so the high-quality perovskite film is a prerequisite to achieve a high efficiency.

Buonassisi et al. used solution-processing and vaporassisted techniques to synthesize pure phase $\left(\mathrm{CH}_{3} \mathrm{NH}_{3}\right)_{3} \mathrm{Bi}_{2} \mathrm{I}_{9}$ perovskite (Fig. 12a) [164]. They found that the crystal structure of the pure phase $\left(\mathrm{CH}_{3} \mathrm{NH}_{3}\right)_{3} \mathrm{Bi}_{2} \mathrm{I}_{9}$ was composed of alternate $\mathrm{MA}^{+}$and $\mathrm{Bi}_{2} \mathrm{I}_{9}{ }^{3-}$ groups (Fig. 13b). The perovskite films prepared by this method were densely packed with high coverage and also showed excellent stability (Fig. 12c, d). The vapor processed films also got a longer PL decay time [164].

Very recently, Zhang et al. made a breakthrough in experimental method of fabricating high-quality $\left(\mathrm{CH}_{3} \mathrm{NH}_{3}\right)_{3} \mathrm{Bi}_{2} \mathrm{I}_{9}$ film [165]. They employed a novel solvent-free high-low vacuum deposition (HLVD) procedure to obtain smooth, compact and pinhole-free polygonal crystal $\left(\mathrm{CH}_{3} \mathrm{NH}_{3}\right)_{3} \mathrm{Bi}_{2} \mathrm{I}_{9}$ film and then fabricated heterojunction solar cells. The HLVD approach proposed here is a two-step alternate vacuum deposition of $\mathrm{BiI}_{3}$ and MAI under high- and lowvacuum, which can be further transformed to the resultant $\left(\mathrm{CH}_{3} \mathrm{NH}_{3}\right)_{3} \mathrm{Bi}_{2} \mathrm{I}_{9}$ perovskite. With the approximate $300 \mathrm{~nm}$ $\left(\mathrm{CH}_{3} \mathrm{NH}_{3}\right)_{3} \mathrm{Bi}_{2} \mathrm{I}_{9}$ film as the light absorption layer to construct the planar heterojunction PSCs, they not only achieved a high PCE of $1.64 \%$ and a high EQE close to $60 \%$, but also obtained a good stability during the measurement of 15 weeks, demonstrating the potential of $\left(\mathrm{CH}_{3} \mathrm{NH}_{3}\right)_{3} \mathrm{Bi}_{2} \mathrm{I}_{9}$ for highly efficient and stable solar cells.

In subsequent research, Ran et al. synthesized a smooth, uniform and pinhole-free $\left(\mathrm{CH}_{3} \mathrm{NH}_{3}\right)_{3} \mathrm{Bi}_{2} \mathrm{I}_{9}$ thin film with a novel two-step evaporation spin coating film fabrication strategy (Fig. 13a, b) [166]. They further optimized film formation conditions by comparing the integrated current from the IPCE measurements and formed compact $\left(\mathrm{CH}_{3} \mathrm{NH}_{3}\right)_{3} \mathrm{Bi}_{2} \mathrm{I}_{9}$ thin films. By taking advantage of the optimized compact thin film, they manufactured inverted
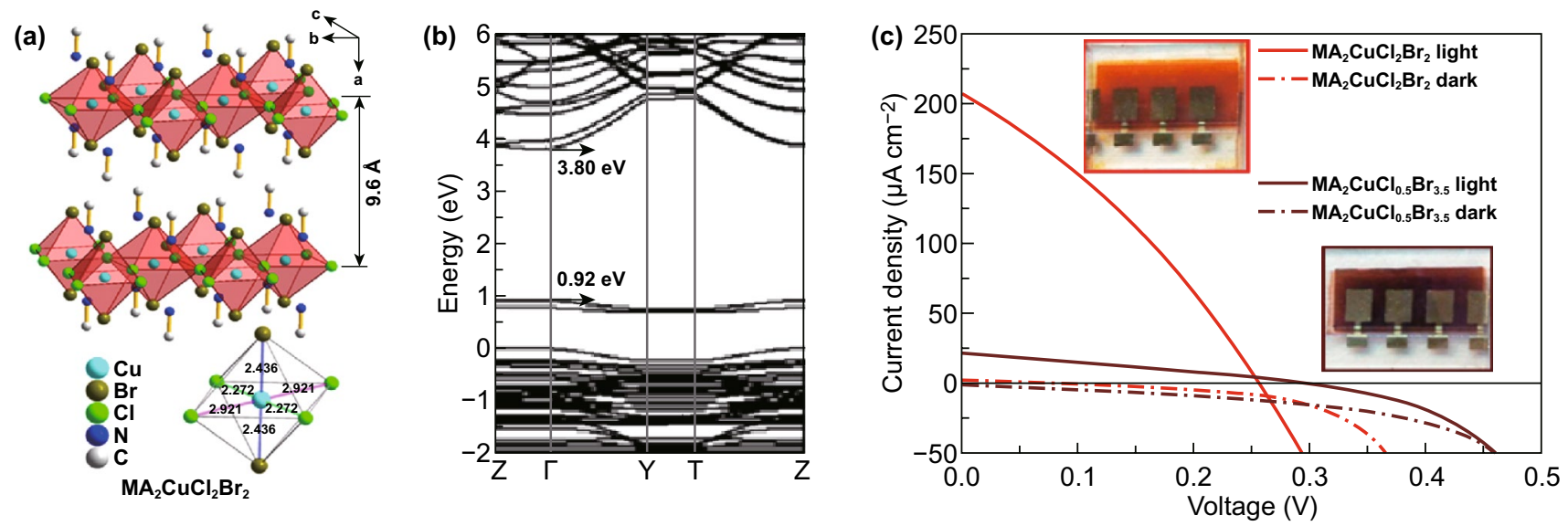

Fig. 10 a Crystal structure of $\mathrm{MA}_{2} \mathrm{CuCl}_{2} \mathrm{Br}_{2}$, showing the alternation of organic and inorganic layers and the $\mathrm{Cu}-\mathrm{X}$ bond lengths in the inorganic framework. b Electronic band structure of $\mathrm{MA}_{2} \mathrm{CuClBr}_{3}$ investigated by DFT simulation. c $J-V$ curve of solar cells sensitized with $\mathrm{MA}_{2} \mathrm{CuCl}_{2} \mathrm{Br}_{2}$ (red) and $\mathrm{MA}_{2} \mathrm{CuCl}_{0.5} \mathrm{Br}_{3.5}$ (brown) under 1 sun of light illumination. The red and brown dashed lines represent dark current. Reproduced with permission from Ref. [63] 

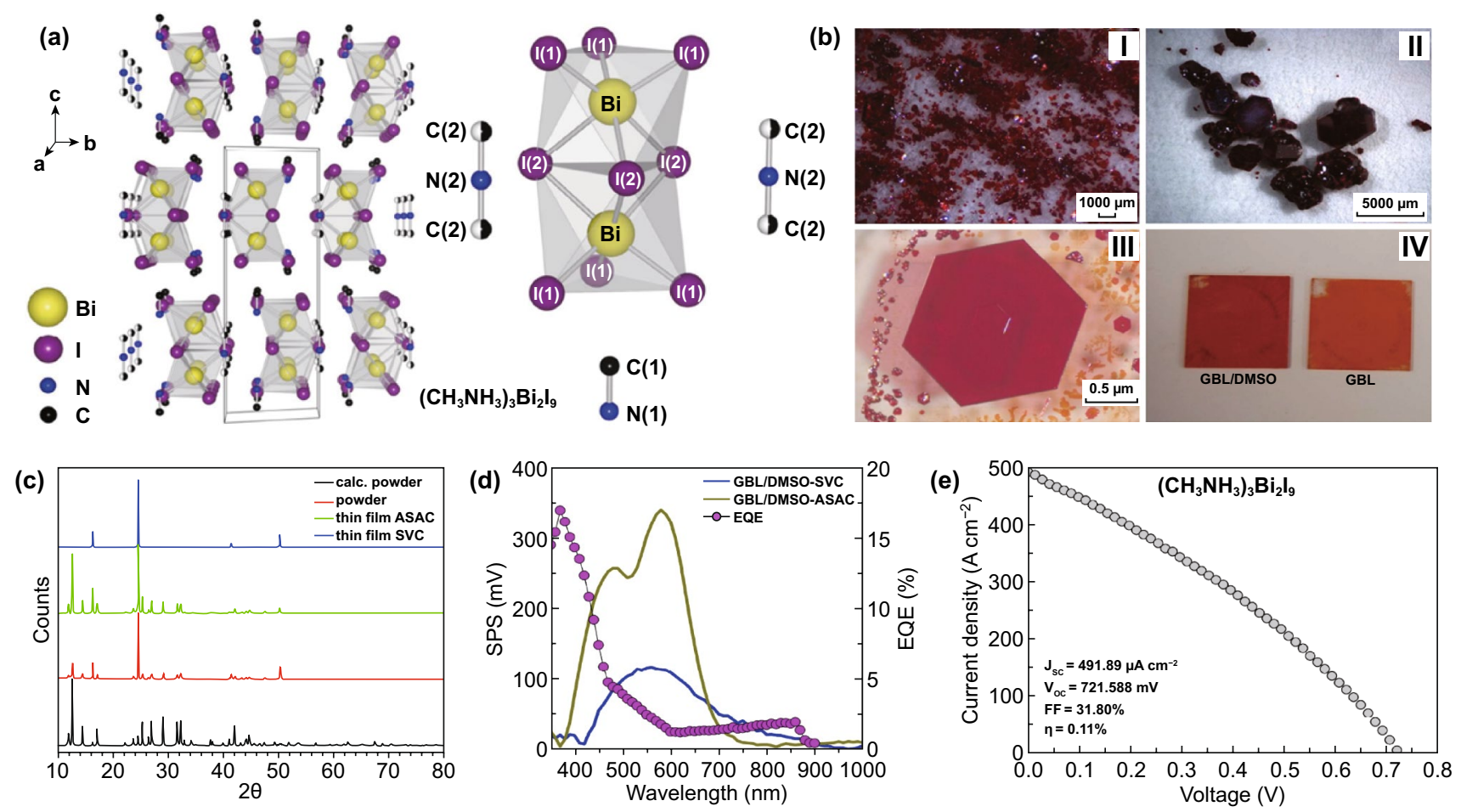

Fig. 11 a A representation of the layered structure of $\left(\mathrm{CH}_{3} \mathrm{NH}_{3}\right)_{3} \mathrm{Bi}_{2} \mathrm{I}_{9}$, characterized by isolated $\left[\mathrm{Bi}_{2} \mathrm{I}_{9}\right]^{3-}$ anions and two crystallographic inequivalent $\mathrm{CH}_{3} \mathrm{NH}_{3}{ }^{+}$cations. b Small single-crystal powder grown from $\mathrm{Bi}_{2} \mathrm{O}_{3}$ and $\mathrm{CH}_{3} \mathrm{NH}_{3} \mathrm{I}$ in concentrated $\mathrm{HI}$ (I-III). And (CH $\left.\mathrm{CH}_{3}\right)_{3} \mathrm{Bi}_{2} \mathrm{I}_{9}$ thin films deposited by the antisolvent assisted crystallization method (ASAC) using GBL/DMSO and GBL as solvents and chlorobenzene as antisolvent (IV). c Diffraction patterns of $\left(\mathrm{CH}_{3} \mathrm{NH}_{3}\right)_{3} \mathrm{Bi}_{2} \mathrm{I}_{9}$ thin films, powder and simulated powder from single-crystal XRD measurements. $\mathbf{d}$ EQE of a typical FTO/TiO $/\left(\mathrm{CH}_{3} \mathrm{NH}_{3}\right)_{3} \mathrm{Bi}_{2} \mathrm{I}_{9} /$ spiro-MeOTAD/Au device. e $J-V$ scanning of the best-forming device. Reproduced with permission from Ref. [163]

planar heterojunction photovoltaic device with a PCE of $0.39 \%$ and a high $V_{\text {oc }}$ of $0.83 \mathrm{~V}$, which exhibited the lowest loss-in-potential to date in $\left(\mathrm{CH}_{3} \mathrm{NH}_{3}\right)_{3} \mathrm{Bi}_{2} \mathrm{I}_{9}$-based solar cells (Fig. 13c-e). However, there still existed a slight hysteresis effect in the $J-V$ curves [166]. This work certificates that the film morphology engineering is crucial for improving the optoelectronic performance of Bi-based hybrid perovskites solar cells.

Additionally, Park et al. confirmed more efficient solar cells with MA cations replaced by Cs cations in Bi-based perovskite [59]. On the basis of that research, Johansson et al. introduced a bismuth halide as the light absorber with the chemical composition of $\mathrm{Cs}_{3} \mathrm{Bi}_{2} \mathrm{I}_{9}$ in their solar cells [167]. The morphology of the $\mathrm{Cs}_{3} \mathrm{Bi}_{2} \mathrm{I}_{9}$ sample showed hexagonal flakes with size up to $2 \mu \mathrm{m}$, which were vertically organized and penetrated into $\mathrm{TiO}_{2}$. The $\mathrm{TiO}_{2}$ can be seen in the gaps between the large flakes due to the unique perovskite morphology and stacking mode. The best-performing device based on $\mathrm{Cs}_{3} \mathrm{Bi}_{2} \mathrm{I}_{9}$ showed a $J_{\text {sc }}$
$2.2 \mathrm{~mA} \mathrm{~cm}^{-2}$, but lower $V_{\mathrm{oc}}$ and $\mathrm{FF}$ values generated a PCE of $0.4 \%$ [167].

Although researchers have got some successful examples of Bi-based perovskites, the performances of their perovskite solar cells are still universally low due to the intrinsic large bandgaps, large carrier effective masses, large exciton binding energies, more internal defects and defect intolerance. Design of new Bi-based lead-free perovskite materials avoiding above issues on the basis of theoretical calculation is critical for further development of high-performance solar cells. In addition, accurate control of formation rate with facile process technologies (e.g., moisture assisted growth, vacuum deposition, hot spin coating, and so forth) to improve the film quality of Bi-based perovskite allows for an improved performance of Bi-based lead-free perovskite devices. Here, we summarize the current research results of Bi-based PSCs in Table 2 [59, 161, 163-170].

$\mathrm{Sb}$ is in the same element group as Bi with low toxicity although it is a heavy metal [171, 172]. Halide perovskites 
(a) $\mathrm{Bil}_{3}$ in $\mathrm{DMF}$

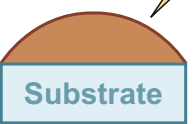
$20^{\circ} \mathrm{C}$

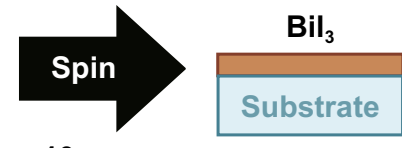

$10 \mathrm{~s}$

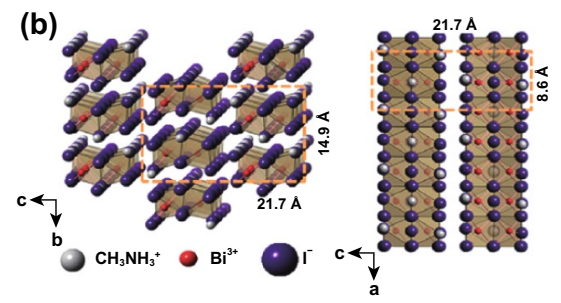

(c)

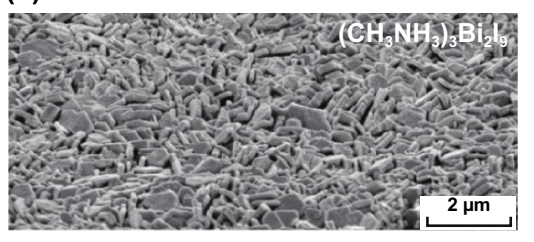

$100{ }^{\circ} \mathrm{C}$

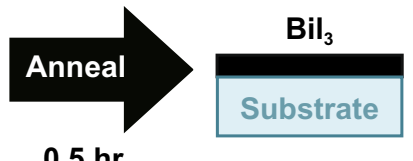

$20^{\circ} \mathrm{C}$

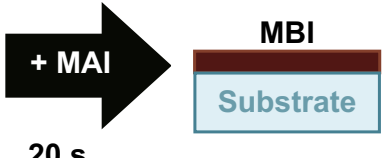

$20 \mathrm{~s}$

(d)

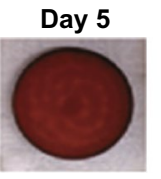

Day 13

Day 26
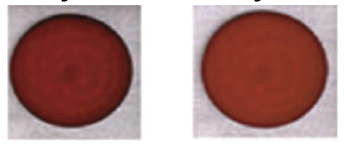

$\left(\mathrm{CH}_{3} \mathrm{NH}_{3}\right)_{3} \mathrm{Bi}_{2} \mathrm{I}_{9}$

Fig. 12 a Schematic diagram of $\left(\mathrm{CH}_{3} \mathrm{NH}_{3}\right)_{3} \mathrm{Bi}_{2} \mathrm{I}_{9}$ solution-assisted process. b Crystal structure and (001) view of $\left(\mathrm{CH}_{3} \mathrm{NH}_{3}\right)_{3} \mathrm{Bi}_{2} \mathrm{I}_{9}$. c SEM image of $\left(\mathrm{CH}_{3} \mathrm{NH}_{3}\right)_{3} \mathrm{Bi}_{2} \mathrm{I}_{9}$ thin film. d Stability measurement of $\left(\mathrm{CH}_{3} \mathrm{NH}_{3}\right)_{3} \mathrm{Bi}_{2} \mathrm{I}_{9}$ in air. Reproduced with permission from Ref. [164]
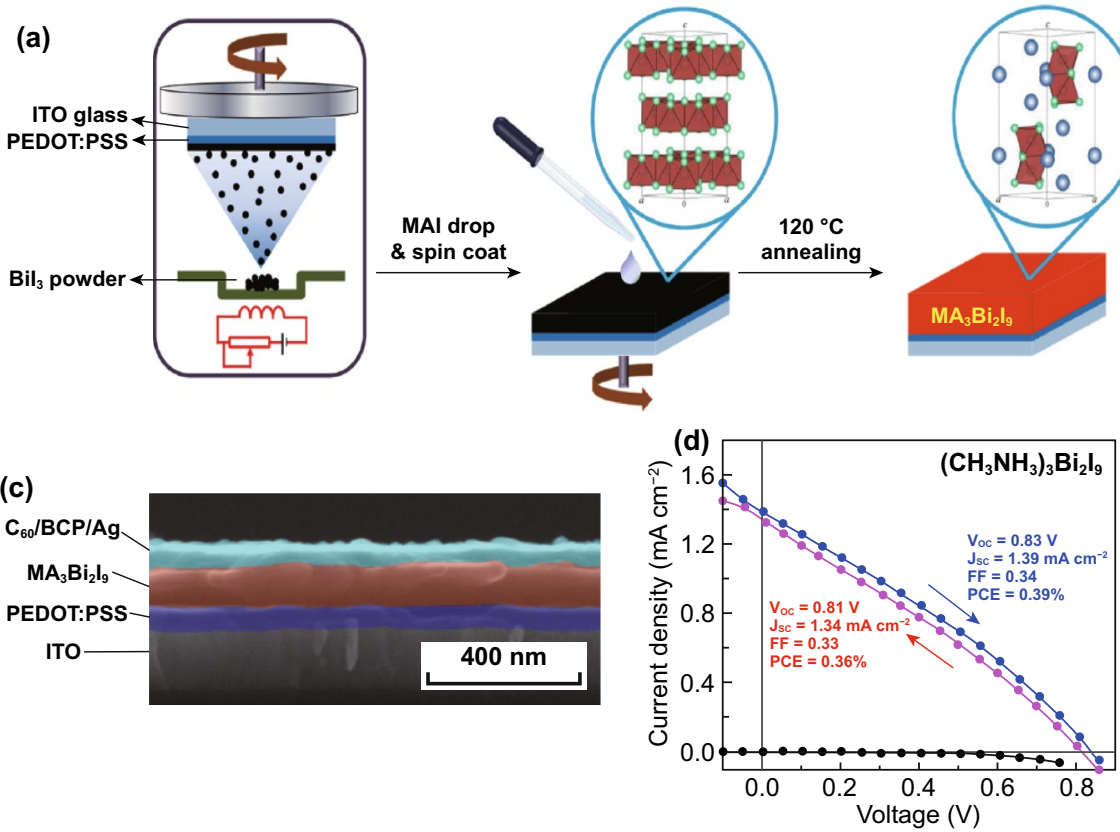
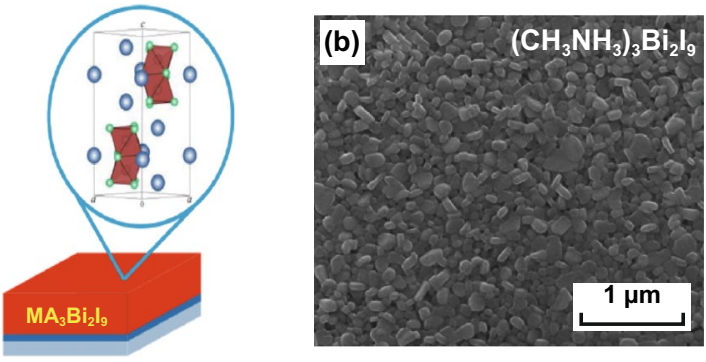

(c)

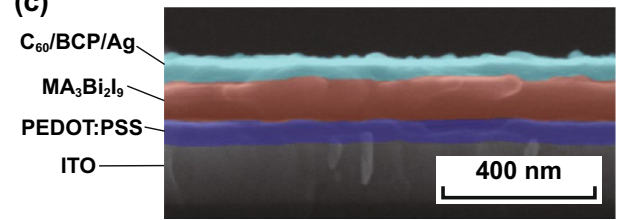

(e)

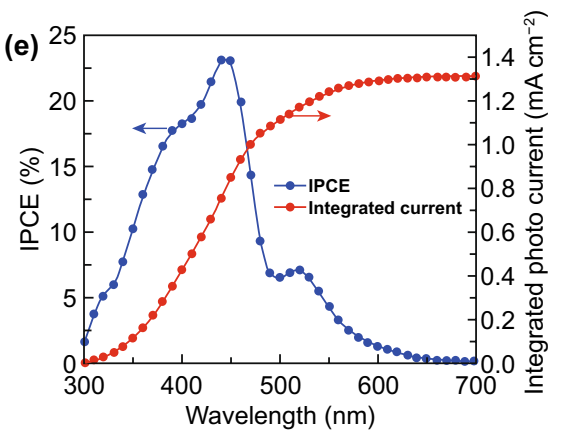

Fig. 13 a Fabrication procedure and b SEM image of $\left(\mathrm{CH}_{3} \mathrm{NH}_{3}\right)_{3} \mathrm{Bi}_{2} \mathrm{I}_{9}$ film. c Cross-sectional SEM image of $\left(\mathrm{CH}_{3} \mathrm{NH}_{3}\right)_{3} \mathrm{Bi}_{2} \mathrm{I}_{9}$-based solar cells. d Forward and backward scanning and e IPCE spectra of the best-forming device. Reproduced with permission from Ref. [166]

based on group-VA cations of $\mathrm{Sb}^{3+}$ are promising candidates due to the same lone-pair $n s^{2}$ state as $\mathrm{Pb}^{2+}$. Through a joint experimental and theoretical study, researchers found that a OD structure was formed in Sb-based perovskite, which was similar to Bi, but with lower exciton binding energies. Mitzi et al. reported a highly oriented Sb-based perovskite thin film by a two-step deposition approach. Large grain $(>1 \mu \mathrm{m})$ and continuous thin films of the lead-free perovskite $\mathrm{Cs}_{3} \mathrm{Sb}_{2} \mathrm{I}_{9}$ were achieved by annealing the evaporated $\mathrm{CsI}$ in $\mathrm{SbI}_{3}$ vapor at $300{ }^{\circ} \mathrm{C}$ for $10 \mathrm{~min}$, and the $\langle 111\rangle$-stacked layered perovskite structure was convinced by the $\mathrm{X}$-ray diffraction (XRD) patterns [62]. The layered inorganic $\mathrm{Cs}_{3} \mathrm{Sb}_{2} \mathrm{I}_{9}$ has a bandgap of $2.05 \mathrm{eV}$, measured by ultraviolet photoelectron spectroscopy, inverse photoemission spectroscopy and X-ray photoelectron spectroscopy (XPS), which showed similar absorption as high as $\mathrm{CH}_{3} \mathrm{NH}_{3} \mathrm{PbI}_{3}$. The DFT calculations 
indicated $\mathrm{Cs}_{3} \mathrm{Sb}_{2} \mathrm{I}_{9}$ has a nearly direct bandgap in consideration of less than $0.02 \mathrm{eV}$ difference between the direct and indirect bandgaps. Then Mitzi et al. prepared PSCs with the $\mathrm{Cs}_{3} \mathrm{Sb}_{2} \mathrm{I}_{9}$ material, which produced a low PCE of less than $<1 \%$, a significant hysteresis effect, and an enhanced air stability compared to $\mathrm{CH}_{3} \mathrm{NH}_{3} \mathrm{PbI}_{3}$ counterpart [62].

Kirchartz et al. presented solution-treated trivalent antimony perovskite $\left(\mathrm{CH}_{3} \mathrm{NH}_{3}\right)_{3} \mathrm{Sb}_{2} \mathrm{I}_{9}$ and fabricated a planar heterojunction solar cell with this compound (Fig. 14a-c), yielding a PCE of $\sim 0.5 \%$, a decent FF of $55 \%$, a $V_{\text {oc }}$ of $0.89 \mathrm{~V}$ and a low photocurrent density of $1.0 \mathrm{~mA} \mathrm{~cm}^{-2}$ [173]. They determined a peak absorption coefficient $(\alpha)$ of $\sim 10^{5} \mathrm{~cm}^{-1}$ and an optical bandgap of $2.14 \mathrm{eV}$ for amorphous $\left(\mathrm{CH}_{3} \mathrm{NH}_{3}\right)_{3} \mathrm{Sb}_{2} \mathrm{I}_{9}$ films by photothermal deflection spectroscopy. The PL was observed at $1.58 \mathrm{eV}$, and the Urbach tail energy of this amorphous composite was $62 \times 10^{-3} \mathrm{eV}$, demonstrating a substantial amount of energetic disorder [173]. The wide optical bandgap and energetic disorder may be the source of low photocurrent densities. In a word, this type of 0D dimer-phase trivalent antimony perovskite suffers from intrinsic problems including a low-symmetry induced indirect bandgap, strong quantumconfinement effect caused oversized gap values, and inferior hopping-like carrier transport, thus it is unfavorable for photovoltaic applications with extremely low PCEs of less than $0.5 \%$ obtained $[62,173]$.

The 2D layered phase can partially circumvent the abovementioned problems and is expected to show a direct bandgap, smaller optical bandgap and good in-layer carrier transport properties. Recently, with the introduction of small-size $\mathrm{Rb}^{+}$and $\mathrm{NH}_{4}{ }^{+}$as the A-site cations, 2D layered phases of $\mathrm{Rb}_{3} \mathrm{Sb}_{2} \mathrm{I}_{9}$ [174] and $\left(\mathrm{NH}_{4}\right)_{3} \mathrm{Sb}_{2} \mathrm{I}_{9}$ [175] have been realized using low-temperature solution processing. Zhou et al. performed comprehensive chemical composition engineering of the precursor solutions by incorporating $\mathrm{MACl}$ into the mixture of $\mathrm{SbI}_{3}$ and MAI, and successfully realized a phase transformation from the $0 \mathrm{D}$ dimer phase to the $2 \mathrm{D}$ layered one [176]. As displayed in Fig. 14d-j, the presence of the 2D layered phase was demonstrated by X-ray diffraction measurements, optical absorbance spectroscopy, and direct comparison of the experimental data with theoretical calculations. With high-quality films of the 2D layered

Table 2 Photovoltaic parameters of PSCs based on various Bi-based perovskite absorbers

\begin{tabular}{|c|c|c|c|c|c|c|c|}
\hline Preparation process & Absorber & Device architecture & $V_{\mathrm{oc}}(\mathrm{V})$ & $J_{\mathrm{sc}}\left(\mathrm{mA} \mathrm{cm}^{-2}\right)$ & $\mathrm{FF}(\%)$ & PCE $(\%)$ & References \\
\hline One-step spin coating & $\mathrm{Cs}_{3} \mathrm{Bi}_{2} \mathrm{I}_{9}$ & $\begin{array}{l}\mathrm{c}-\mathrm{TiO}_{2} / \mathrm{m}-\mathrm{TiO}_{2} / \text { perovskite/ } \\
\mathrm{P} 3 \mathrm{HT} / \mathrm{Ag}\end{array}$ & 0.31 & 0.34 & 38 & 0.40 & {$[165]$} \\
\hline One-step spin coating & $\left(\mathrm{CH}_{3} \mathrm{NH}_{3}\right)_{3} \mathrm{Bi}_{2} \mathrm{I}_{9}$ & $\mathrm{c}-\mathrm{TiO}_{2} /$ perovskite/spiro/Au & 0.72 & 0.49 & 31.8 & 0.11 & {$[161]$} \\
\hline One-step spin coating & $\left(\mathrm{CH}_{3} \mathrm{NH}_{3}\right)_{3} \mathrm{Bi}_{2} \mathrm{I}_{9}$ & $\begin{array}{l}\mathrm{c}-\mathrm{TiO}_{2} / \mathrm{m}-\mathrm{TiO}_{2} / \text { perovskite/ } \\
\text { spiro-MeOTAD/Au }\end{array}$ & 0.68 & 0.38 & 88 & 0.22 & [166] \\
\hline One-step spin coating & $\left(\mathrm{CH}_{3} \mathrm{NH}_{3}\right)_{3} \mathrm{Bi}_{2} \mathrm{I}_{9}$ & $\begin{array}{l}\text { c-TiO } / 2 / \mathrm{m}^{-\mathrm{TiO}_{2}} / \text { perovskite/ } \\
\text { spiro-MeOTAD/Ag }\end{array}$ & 0.68 & 0.52 & 33 & 0.12 & [59] \\
\hline One-step spin coating & $\mathrm{Cs}_{3} \mathrm{Bi}_{2} \mathrm{I}_{9}$ & $\begin{array}{l}\text { c- } \mathrm{TiO}_{2} / \mathrm{m}^{-\mathrm{TiO}_{2}} / \text { perovskite/ } \\
\text { spiro-MeOTAD/Ag }\end{array}$ & 0.85 & 2.15 & 60 & 1.09 & [59] \\
\hline One-step spin coating & $\left(\mathrm{CH}_{3} \mathrm{NH}_{3}\right)_{3} \mathrm{Bi}_{2} \mathrm{I}_{9} \mathrm{Cl}_{\mathrm{x}}$ & $\begin{array}{l}\text { c-TiO } / 2 / \mathrm{m}^{-\mathrm{TiO}_{2}} / \text { perovskite/ } \\
\text { spiro-MeOTAD/Ag }\end{array}$ & 0.04 & 0.18 & 38 & 0.03 & {$[59]$} \\
\hline One-step spin coating & $\left(\mathrm{CH}_{3} \mathrm{NH}_{3}\right)_{3} \mathrm{Bi}_{2} \mathrm{I}_{9}$ & $\begin{array}{l}\mathrm{c}-\mathrm{TiO}_{2} / \mathrm{m}-\mathrm{TiO}_{2} / \text { perovskite/ } \\
\mathrm{P} 3 \mathrm{HT} / \mathrm{Au}\end{array}$ & 0.35 & 1.157 & 46.4 & 0.19 & {$[167]$} \\
\hline One-step spin coating & $\left(\mathrm{CH}_{3} \mathrm{NH}_{3}\right)_{3} \mathrm{Bi}_{2} \mathrm{I}_{9}$ & $\begin{array}{l}\mathrm{c}-\mathrm{TiO}_{2} / \mathrm{m}-\mathrm{TiO}_{2} / \text { perovskite/ } \\
\text { PIF8-TAA } / \mathrm{Au}\end{array}$ & 0.85 & 1.22 & 73 & 0.71 & {$[168]$} \\
\hline $\begin{array}{l}\text { Two-step evaporation spin } \\
\text { coating }\end{array}$ & $\left(\mathrm{CH}_{3} \mathrm{NH}_{3}\right)_{3} \mathrm{Bi}_{2} \mathrm{I}_{9}$ & $\begin{array}{l}\text { PEDOT:PSS/perovskite/C }{ }_{60} / \\
\text { BCP/Ag }\end{array}$ & 0.83 & 1.39 & 37 & 0.39 & {$[164]$} \\
\hline Two-step thermal evaporation & $\left(\mathrm{CH}_{3} \mathrm{NH}_{3}\right)_{3} \mathrm{Bi}_{2} \mathrm{I}_{9}$ & $\begin{array}{l}\mathrm{c}-\mathrm{TiO}_{2} / \mathrm{m}^{-\mathrm{TiO}_{2}} / \text { perovskite/ } \\
\text { spiro-MeOTAD/Au }\end{array}$ & 0.83 & 3.00 & 79 & 1.64 & {$[163]$} \\
\hline Vapor-assisted solution process & $\left(\mathrm{CH}_{3} \mathrm{NH}_{3}\right)_{3} \mathrm{Bi}_{2} \mathrm{I}_{9}$ & $\begin{array}{l}\mathrm{c}-\mathrm{TiO}_{2} / \mathrm{m}^{-\mathrm{TiO}_{2}} / \text { perovskite/ } \\
\mathrm{P} 3 \mathrm{HT} / \mathrm{Au}\end{array}$ & 1.01 & 4.02 & 78 & 3.17 & [169] \\
\hline One-step spin coating & $\mathrm{Cs}_{3} \mathrm{Bi}_{2} \mathrm{I}_{9}$ & $\begin{array}{l}\text { c- } \mathrm{TiO}_{2} / \text { perovskite/spiro-MeO- } \\
\mathrm{TAD} / \mathrm{Au}\end{array}$ & 0.79 & 4.45 & 50.34 & 1.77 & {$[170]$} \\
\hline One-step spin coating & $\mathrm{Cs}_{3} \mathrm{Bi}_{2} \mathrm{I}_{9}$ & c- $\mathrm{TiO}_{2} /$ perovskite/CuI/Au & 0.86 & 5.78 & 64.38 & 3.20 & {$[170]$} \\
\hline One-step spin coating & $\mathrm{Cs}_{3} \mathrm{Bi}_{2} \mathrm{I}_{9}$ & $\mathrm{c}-\mathrm{TiO}_{2} /$ perovskite/PTAA/Au & 0.83 & 4.82 & 57.49 & 2.30 & {$[170]$} \\
\hline
\end{tabular}


$\mathrm{MA}_{3} \mathrm{Sb}_{2} \mathrm{Cl}_{\mathrm{X}} \mathrm{I}_{9-\mathrm{X}}$ perovskites as light absorbers, they fabricated solar cells with PCEs of over 2\% [176].

So far, the Sb-based perovskites, similar to bismuth ones, still show a limited efficiency. But they own a bright application prospect once their nearly direct bandgap can be adjusted to a suitable value (e.g., doping) that matches with solar spectra. In addition, Sb-based perovskites can form a more stable 2D layered phase, which are better for carrier transport and display improved optoelectronic properties compared with the 0D Bi-based counterparts.

\subsubsection{Chalcogen-Halogen Hybrid Perovskite}

In 2015, Sun et al. proposed the anion-split approach based on first-principles calculation, where dual anions (e.g., halogen and chalcogen anions) were introduced while $\mathrm{Pb}$ was replaced by heterovalent non-toxic elements in order to keep the 3D perovskite structure and the charge neutrality (Fig. 8b, c) [52]. Figure 8b shows the atomic structure of $\mathrm{MABiSe}_{2}$, which maintained the $\mathrm{MAPbI}_{3}$ tetragonal structure with a freely rotating $\mathrm{MA}^{+}$ion according to ab initio molecular dynamics (AIMD) simulations.

Taking Bi as the core element of the B position, Shockley et al. examined a series of $\mathrm{MABiChX}_{2}(\mathrm{Ch}=\mathrm{S}$, Se, Te; $\mathrm{X}=\mathrm{I}, \mathrm{Br}, \mathrm{Cl}$ ) compounds and found that $\mathrm{CH}_{3} \mathrm{NH}_{3} \mathrm{BiSeI}_{2}$ and $\mathrm{CH}_{3} \mathrm{NH}_{3} \mathrm{BiSI}_{2}$ exhibited improved optical absorption and reduced bandgaps (1.3-1.4 eV) over $\mathrm{CH}_{3} \mathrm{NH}_{3} \mathrm{PbI}_{3}$, which were proved to be the optimal value for solar cell absorbers according to the Shockley-Queisser theory (Fig. 8c) [177]. These results suggested that the $3 \mathrm{D} \mathrm{AB}(\mathrm{Ch}, \mathrm{X})_{3}$ perovskites represented by $\mathrm{MABiSI}_{2}$ could be promising candidates of photovoltaic absorbers once they were successfully synthesized. Unfortunately, the subsequent feasibility assessment of the proposed $3 \mathrm{D} \mathrm{AB}(\mathrm{Ch}, \mathrm{X})_{3}$ perovskites by Hong et al. via DFT calculations and solid-state reactions indicated that all of the as-proposed $\mathrm{AB}(\mathrm{Ch}, \mathrm{X})_{3}$ perovskites are thermodynamically unstable [34], and they tend to decompose into ternary and/or binary phases or form nonperovskite phases. Recently, the work of Li et al. has proved the instability of chalcogen-halogen hybrid perovskite through experimental and computational analysis, and they found that $\mathrm{AB}(\mathrm{Ch}$, $\mathrm{X})_{3}$ would be decomposed into a mixture of binary and ternary compounds $\left(\mathrm{Sb}_{2} \mathrm{~S}_{3}\right.$ and $\left.\mathrm{MA}_{3} \mathrm{Sb}_{2} \mathrm{I}_{9}\right)$ soon after being synthesized [178]. Upon inspection, the target perovskites are impossible to be successfully synthesized as designed.
Finally, the researcher had to draw a conclusion that it may be a huge challenge to synthesize the proposed $\mathrm{AB}(\mathrm{Ch}, \mathrm{X})_{3}$ perovskites due to their thermodynamic instability, which means there is still a long way for $\mathrm{AB}(\mathrm{Ch}, \mathrm{X})_{3}$ perovskites to go for further applications in perovskite photovoltaic devices.

\subsubsection{OD Perovskite Derivative $A_{2} B X_{6}$}

Previous analysis indicated that it is feasible to replace $\mathrm{Pb}$ of lead halide perovskites with tetravalent B(IV) substitutes. To accommodate the heterovalent substitute, the chemical formula needs to turn into $\mathrm{A}_{2} \mathrm{BX}_{6}$, which is derived from its $\mathrm{ABX}_{3}$ perovskite structure by removing half of $\mathrm{B}$-site cations. Because of the large charge difference between them, the $\mathrm{A}_{2} \mathrm{BX}_{6}$ perovskite variant is sometimes referred to as the $\mathrm{A}_{2} \mathrm{~B} \square \mathrm{X}_{6}$-type vacant ordered double perovskite [86, 179-181]. The B-site vacancies (denoted as $\square$ ) and the remaining $\mathrm{B}$-site cations generally adopt a rock salt arrangement in the $\mathrm{A}_{2} \mathrm{BX}_{6}$ perovskite structure. Due to the absence of connectivity between the $\left[\mathrm{BX}_{6}\right]$ octahedra, the $\mathrm{A}_{2} \mathrm{BX}_{6}$ perovskite variants are actually $0 \mathrm{D}$ nonperovskites despite researchers still would like to call them perovskites. The optoelectronic properties of the $\mathrm{A}_{2} \mathrm{BX}_{6}$-type perovskite materials are rather different from those of $3 \mathrm{D} \mathrm{ABX}_{3}$ $(\mathrm{B}=\mathrm{Pb}, \mathrm{Sn}$, and $\mathrm{Ge})$ perovskites due to the isolated $\left[\mathrm{BX}_{6}\right]$ octahedra in $\mathrm{A}_{2} \mathrm{BX}_{6}$ compounds. Among the $\mathrm{A}_{2} \mathrm{BX}_{6}$ compounds [182-185], $\mathrm{A}_{2} \mathrm{SnI}_{6}$ (A=Cs, MA) [182, 183] and $\mathrm{Cs}_{2} \mathrm{TiBr}_{6}[181,182]$ have been investigated for photovoltaic applications. These $\mathrm{A}_{2} \mathrm{BX}_{6}$ compounds show intrinsic n-type conductivity. Compared to $3 \mathrm{D} \mathrm{CsSnI}_{3}$ with better defect tolerance, the $\mathrm{A}_{2} \mathrm{BX}_{6}$-type materials with I vacancies and Sn interstitial defects have deeper levels in their bandgaps owing to the strong covalent nature of the $\left[\mathrm{SnI}_{6}\right]$ octahedron, seriously impacting optoelectronic performances of devices.

The vacancy-ordered $\mathrm{A}_{2} \mathrm{M}(\mathrm{IV}) \mathrm{X}_{6}$ double perovskites was found to have suitable direct bandgaps. In 2016, Cao et al. found the spontaneous oxidative conversion of unstable $\mathrm{CsSnI}_{3}$ to air-stable $\mathrm{Cs}_{2} \mathrm{SnI}_{6}$ in air [183]. The $\mathrm{Cs}_{2} \mathrm{SnI}_{6}$ perovskite was adopted as the light absorber layer of leadfree perovskite solar cell for the first time due to its small bandgap of $1.48 \mathrm{eV}$ and high absorption coefficient, showing a PCE of about $1 \%$ with a $V_{\mathrm{oc}}$ of $0.51 \mathrm{~V}$ and a $J_{\mathrm{sc}}$ of $5.41 \mathrm{~mA} \mathrm{~cm}^{-2}$ after optimizing the perovskite film thickness. 
(a)

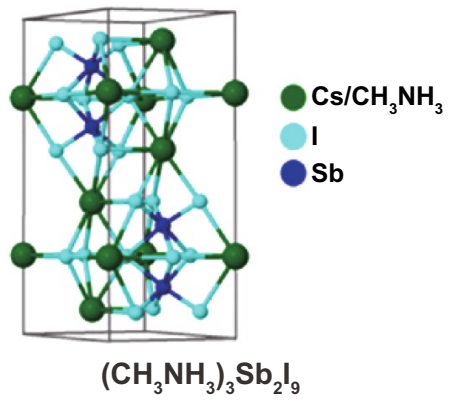

(d)

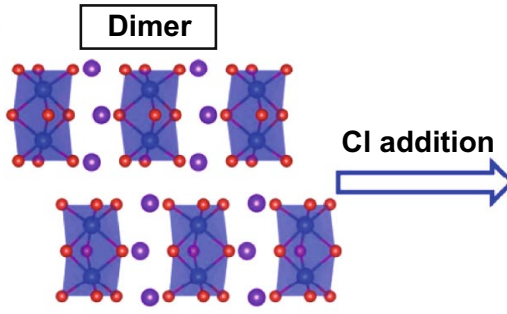

$\mathrm{A}_{3} \mathrm{Sb}_{2} \mathrm{I}_{9}$

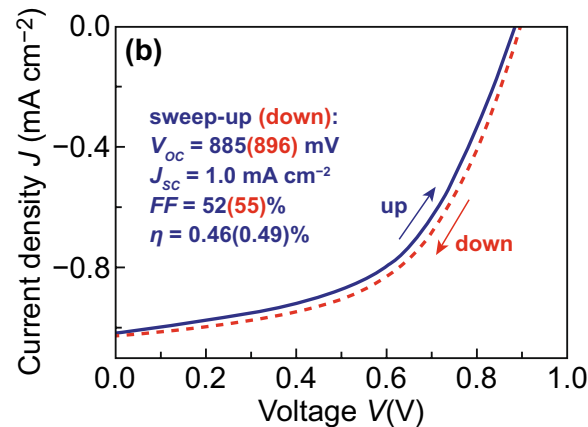

Layer

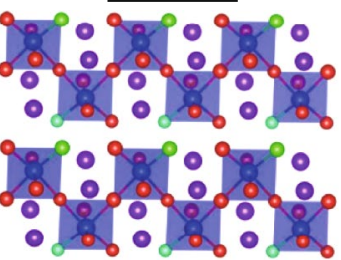

$\mathrm{A}_{3} \mathrm{Sb}_{2} \mathrm{Cl}_{\mathbf{x}} \mathrm{I}_{-\mathrm{x}}$

(g)

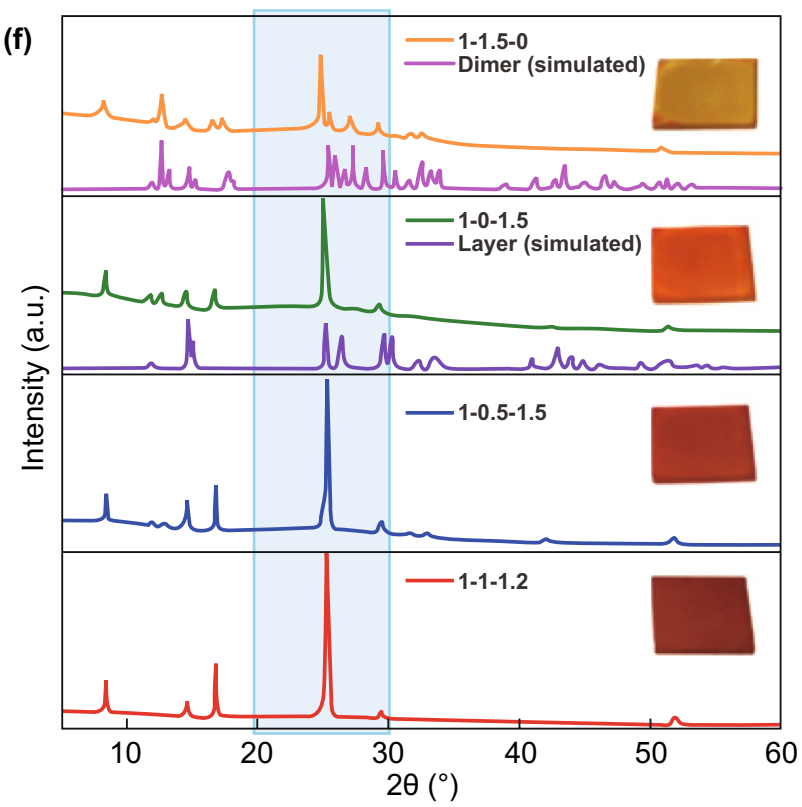

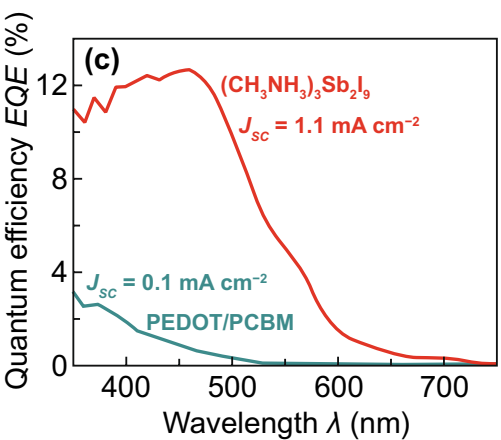

(e)

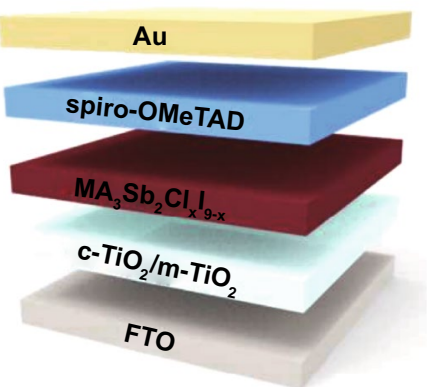

(h)
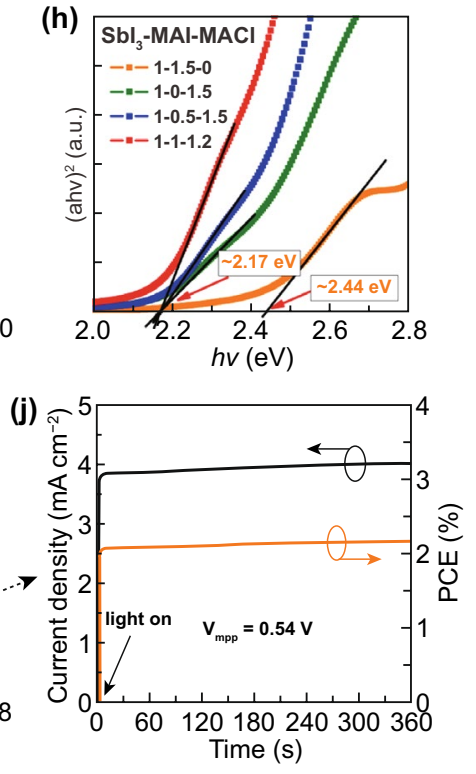

Fig. 14 a Crystal structure of $\left(\mathrm{CH}_{3} \mathrm{NH}_{3}\right)_{3} \mathrm{Sb}_{2} \mathrm{I}_{9}$ (space group $\left.\mathrm{P}_{3} / m m c\right)$. b Illuminated $J-V$ curves of $\left(\mathrm{CH}_{3} \mathrm{NH}_{3}\right)_{3} \mathrm{Sb}_{2} \mathrm{I}_{9}$ solar cell measured with forward and backward scanning with a rate of $0.1 \mathrm{~V} \mathrm{~s}^{-1}$. $\mathbf{c}$ EQE measurement of the $\left(\mathrm{CH}_{3} \mathrm{NH}_{3}\right)_{3} \mathrm{Sb}_{2} \mathrm{I}_{9}$ solar cell compared to the reference device of ITO/PEDOT/PCBM/ZnO-NP/Al. Reproduced with permission from Ref. [173] d Schematic plot of the Cl doping-induced transformation from the 0D dimer phase of $\mathrm{A}_{3} \mathrm{Sb}_{2} \mathrm{I}_{9}$ to the 2D layered phase of $\mathrm{A}_{3} \mathrm{Sb}_{2} \mathrm{Cl}_{\mathrm{X}} \mathrm{I}_{9-\mathrm{X}}$. e Schematic structure of the as-fabricated PSC. $\mathbf{f}$ XRD patterns of the films deposited from precursors containing $\mathrm{SbI}_{3}$, MAI, and $\mathrm{MACl}$ with molar ratios of 1:1.5:0, 1:0:1.5, 1:0.5:1.5, and 1:1:1.2. $\mathbf{g}$ Measured UV-vis absorbance spectra of the four types of films. $\mathbf{h}$ Tauc plots of the absorption coefficients for evaluating the bandgap values of the pureiodine perovskites $\mathrm{MA}_{3} \mathrm{Sb}_{2} \mathrm{I}_{9}$ and Cl-containing mixed-halide perovskites $\mathrm{MA}_{3} \mathrm{Sb}_{2} \mathrm{Cl}_{\mathrm{X}} \mathrm{I}_{9-\mathrm{X}} . \mathbf{i} J-V$ curves for the devices fabricated with the four kinds of perovskite films. $\mathbf{j}$ Steady-state photocurrent output for the device based on 1-1-1.2 film at the maximum power point (red circle). Maximum power point voltage $\mathrm{V}_{\mathrm{mpp}}$ is equal to $0.54 \mathrm{~V}$. Reproduced with permission from Ref. [176]

Soon after, Cao et al. synthesized $\mathrm{Cs}_{2} \mathrm{SnI}_{6}$ powder with a modified solution process and fabricated mesoporous solar cells [183]. They achieved a PCE of $0.96 \%$ by carefully controlling $\mathrm{ZnO}$ nanorod length and pore size. Chang et al. reported a new material $\mathrm{Cs}_{2} \mathrm{SnI}_{6-\mathrm{x}} \mathrm{Br}_{\mathrm{x}}$ with mixed halides which provided desired bandgaps of $\sim 1.3$ to $\sim 2.9 \mathrm{eV}$ suitable 
for solar cells via simple tune of $\mathrm{Br}$ component. They applied this perovskite material as light absorbing layer to PSCs and achieved an optimal conversion efficiency of $2.1 \%$ as $\mathrm{x}=2$ [184]. Recently, $\mathrm{Cs}_{2} \mathrm{TiBr}_{6}$ thin films were prepared through a facile low-temperature vapor-based method and then incorporated into planar heterojunction PSCs [185]. Subsequently, Chen et al. also demonstrated that the $\mathrm{Cs}_{2} \mathrm{TiBr}_{6}$ thin film has a suitable bandgap of $1.8 \mathrm{eV}$, a long and balanced carrier diffusion length, and an appropriate energy level, with which solar cells produced an efficiency of up to $3.3 \%$ [186].

\subsubsection{Double Perovskite $A_{2} B(I) B(I I I) X_{6}$}

Lead-free halide double perovskites materials were known as "elpasolites" many years ago, whose name was from the mineral $\mathrm{K}_{2} \mathrm{NaAlF}_{6}$. In 2016, Giustino et al. counted the elements belonging to halide elpasolites [27]. As shown in Fig. 15, it can be clearly seen that 7 elements can occupy the A-site, and 8 elements including $\mathrm{NH}_{3}{ }^{+}$can occupy the $\mathrm{B}(\mathrm{I})$ site and 34 elements can occupy the $\mathrm{B}$ (III) site, while 5 elements containing the cyanide $\mathrm{CN}^{-}$are able to occupy the $\mathrm{X}$ site. Although many of these compounds are available, it requires to satisfy some prerequisites for photovoltaic applications, such as suitable bandgap, high carrier mobility, low defect state and good thermodynamic stability. Through the element selection of B(I) site and B(III) site in the double perovskite, the bandgap can be well adjusted and thus exhibits excellent optoelectronic properties. For example, $\mathrm{Cs}_{2} \mathrm{NaBiI}_{6}$ has a direct bandgap of $1.66 \mathrm{eV}$. Through element adjustment, $\mathrm{Cs}_{2} \mathrm{AgInBr}_{6}$ with a direct bandgap of $1.50 \mathrm{eV}$ and $\mathrm{Cs}_{2} \mathrm{InSbCl}_{6}$ and $\mathrm{Cs}_{2} \mathrm{InBiCl}_{6}$ with a direct bandgap of about $1.0 \mathrm{eV}$ can be obtained. At present, the researchers have discovered the double perovskite material $\mathrm{Cs}_{2} \mathrm{AgBiBr}_{6}$ possesses with a suitable bandgap of $1.95 \mathrm{eV}$, which can be used as light absorber in solar cells. To date, $\mathrm{Cs}_{2} \mathrm{AgBiBr}_{6}$ is the most commonly used photovoltaic material in leadfree halide double perovskite-based solar cells [187-190]. Bein et al. fabricated $\mathrm{Cs}_{2} \mathrm{AgBiBr}_{6}$ films with a spin coating method and incorporated them into solar cells for the first time in 2017 [187]. After optimizing synthesis conditions, the $\mathrm{Cs}_{2} \mathrm{AgBiBr}_{6}$-based solar cells without encapsulation showed a PCE of $2.43 \%$, a $V_{\text {oc }}$ of exceeding $1 \mathrm{~V}$ and excellent stability in air. The fly in the ointment is that the device has a serious hysteresis effect.

In 2018, Xiao et al. prepared a transparent solution of $\mathrm{Cs}_{2} \mathrm{AgBiBr}_{6}$ in $\mathrm{DMSO}$ and fabricated the $\mathrm{Cs}_{2} \mathrm{AgBiBr}_{6}$ film using spin coating technique by low-pressure-assisted (a)

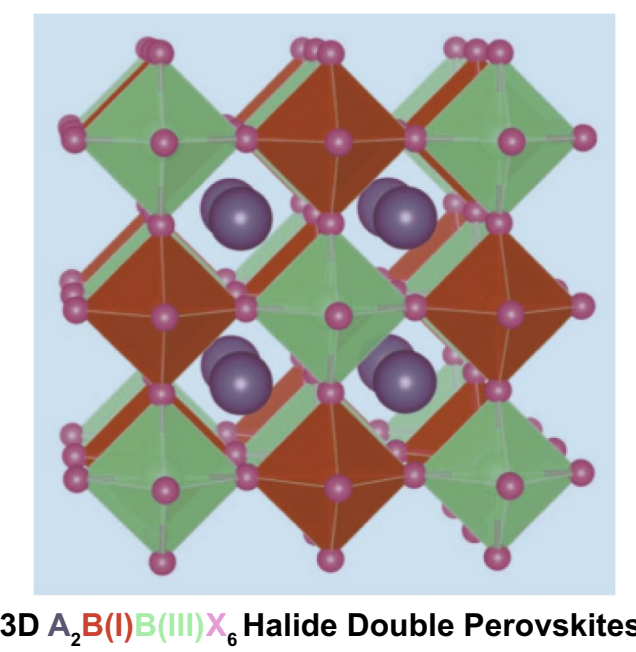

(b)

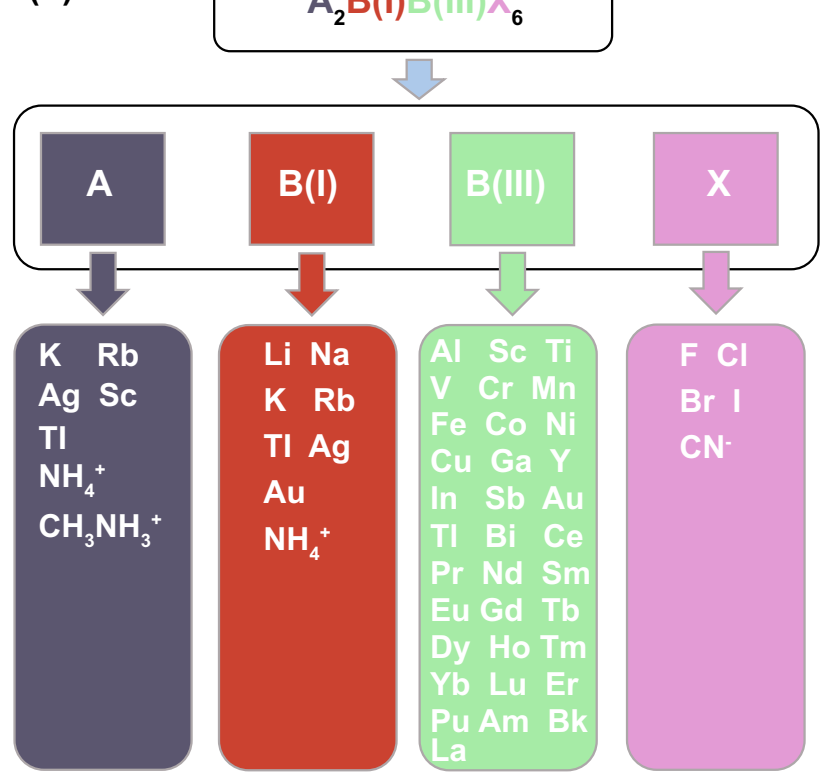

Fig. 15 Crystal structure of double perovskite $\mathrm{A}_{2} \mathrm{~B}(\mathrm{I}) \mathrm{B}(\mathrm{III}) \mathrm{X}_{6}$ (a) and the elements/functional groups that can form double perovskite $\mathrm{A}_{2} \mathrm{~B}(\mathrm{I})$ $\mathrm{B}(\mathrm{III}) \mathrm{X}_{6}$ (b) 

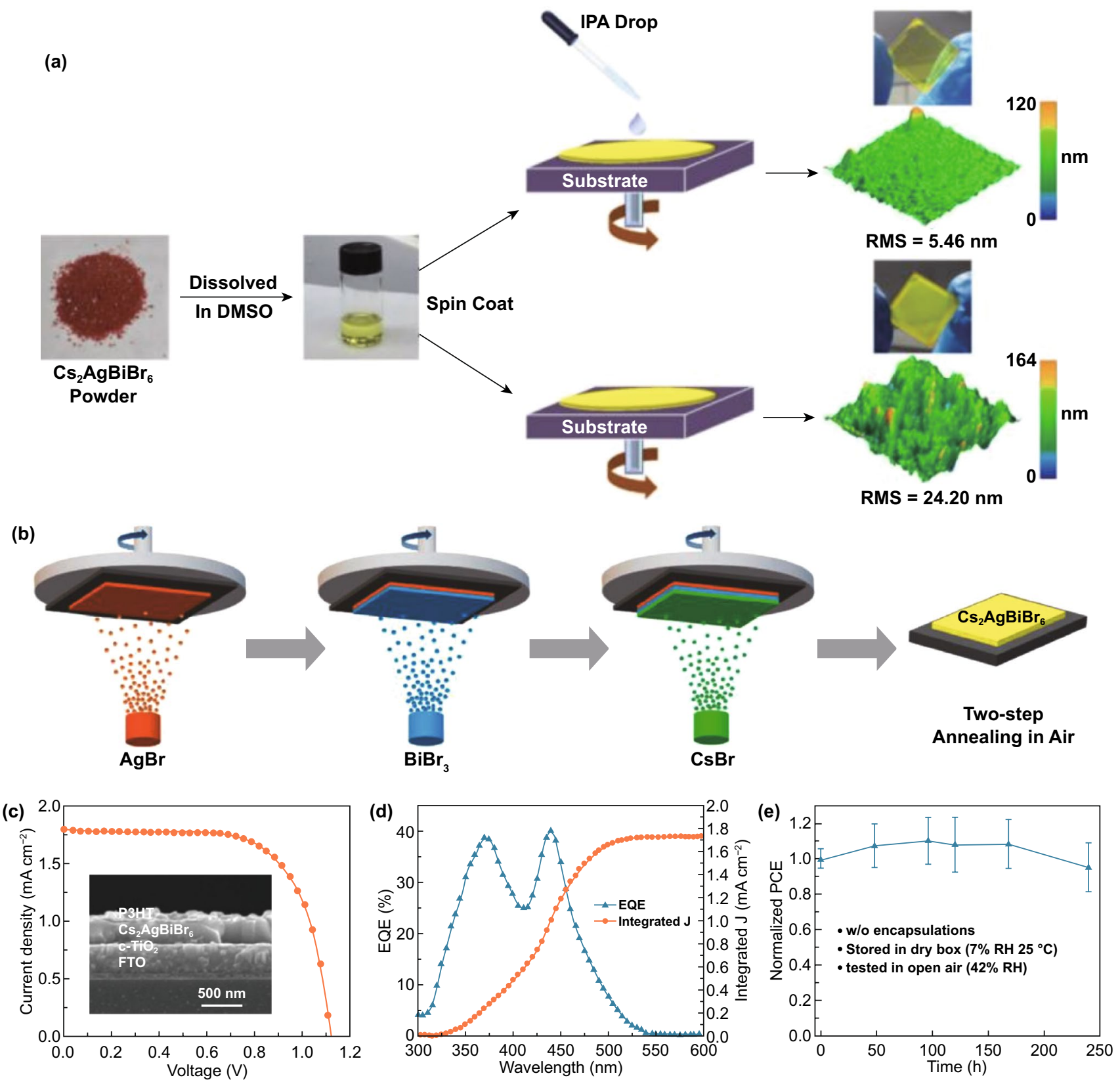

Fig. 16 a Schematic illustration of the spin coating process of $\mathrm{Cs}_{2} \mathrm{AgBiBr}_{4}$ with and without antisolvent dropping program, and the morphology of the as-prepared film. Reproduced with permission from Ref. [189] b Scheme of sequential vapor deposition process. c $J-V$ curve of the optimized solar cell. Inset: Cross-sectional SEM image of the device. d EQE spectrum and the integrated current density with AM $1.5 \mathrm{G}$ photon flux. e Evaluation of long-term stability of the solar cells stored in dry box. Reproduced with permission from Ref. [191]

solution processing under ambient conditions [188]. Differently from the conventional annealing method, the spincoated film was quickly moved to a low-pressure chamber pumped to $20 \mathrm{~Pa}$, where the transparent film gradually turned to light yellow. A smooth morphology was obtained compared with the rough films prepared by conventional annealing methods. Finally, planar heterojunction solar cells were manufactured with optimized $\mathrm{Cs}_{2} \mathrm{AgBiBr}_{6}$ films and P3HT hole transport layer, and a PCE of $1.44 \%$ was achieved. 
Table 3 Photovoltaic parameters of PSCs based on double perovskite $\mathrm{A}_{2} \mathrm{~B}(\mathrm{I}) \mathrm{B}(\mathrm{III}) \mathrm{X}_{6}$ absorbers

\begin{tabular}{|c|c|c|c|c|c|c|c|}
\hline Absorber & $\begin{array}{l}\text { Method for preparing perovskite } \\
\text { film }\end{array}$ & Device architecture & $V_{\mathrm{oc}}(\mathrm{V})$ & $J_{\mathrm{sc}}\left(\mathrm{mA} \mathrm{cm}^{-2}\right)$ & $\mathrm{FF}(\%)$ & PCE $(\%)$ & References \\
\hline $\mathrm{Cs}_{2} \mathrm{AgBiBr}_{6}$ & Spin coating & $\begin{array}{l}\mathrm{c}-\mathrm{TiO}_{2} / \mathrm{m}^{-\mathrm{TiO}_{2}} / \text { perovskite/spiro- } \\
\mathrm{MeOTAD} / \mathrm{Au}\end{array}$ & 0.98 & 3.93 & 63 & 2.43 & [187] \\
\hline $\mathrm{Cs}_{2} \mathrm{AgBiBr}_{6}$ & $\begin{array}{l}\text { Low-pressure-assisted solution pro- } \\
\text { cessing under ambient conditions }\end{array}$ & $\mathrm{SnO}_{2} / \mathrm{Cs}_{2} \mathrm{AgBiBr}_{6} / \mathrm{P} 3 \mathrm{HT} / \mathrm{Au}$ & 1.04 & 1.78 & 78 & 1.44 & {$[188]$} \\
\hline $\mathrm{Cs}_{2} \mathrm{AgBiBr}_{6}$ & $\begin{array}{l}\text { Antisolvent dropping technology } \\
\text { and post-annealing process }\end{array}$ & $\mathrm{Cu}-\mathrm{NiO} / \mathrm{Cs}_{2} \mathrm{AgBiBr}_{6} / \mathrm{C}_{60} / \mathrm{BCP} / \mathrm{Ag}$ & 1.00 & 3.23 & 68.4 & 2.21 & [189] \\
\hline $\mathrm{Cs}_{2} \mathrm{AgBiBr}_{6}$ & $\begin{array}{l}\text { Sequential vapor deposition } \\
\text { method }\end{array}$ & c-TiO 2 /perovskite/P3HT/Au & - & - & - & 1.37 & [191] \\
\hline $\mathrm{Cs}_{2} \mathrm{AgBiBr}_{6}$ & $\begin{array}{l}\text { Spin coating deposition and } \\
\text { annealing at high temperature }\end{array}$ & $\begin{array}{l}\mathrm{c}-\mathrm{TiO}_{2} / \mathrm{m}-\mathrm{TiO}_{2} / \text { perovskite/PTAA/ } \\
\mathrm{Au}\end{array}$ & 1.02 & 1.84 & 67 & 1.26 & {$[190]$} \\
\hline $\mathrm{Cs}_{2} \mathrm{AgBiBr}_{6}$ & $\begin{array}{l}\text { Spin coating deposition and } \\
\text { annealing at high temperature }\end{array}$ & $\begin{array}{l}\mathrm{c}-\mathrm{TiO}_{2} / \mathrm{m}_{-}-\mathrm{TiO}_{2} / \text { perovskite} / \mathrm{PCP}- \\
\mathrm{DTBT} / \mathrm{Au}\end{array}$ & 0.71 & 1.67 & 57 & 0.68 & {$[190]$} \\
\hline $\mathrm{Cs}_{2} \mathrm{AgBiBr}_{6}$ & $\begin{array}{l}\text { Spin coating deposition and } \\
\text { annealing at high temperature }\end{array}$ & $\begin{array}{l}\mathrm{c}-\mathrm{TiO}_{2} / \mathrm{m}-\mathrm{TiO}_{2} / \text { perovskite/spiro- } \\
\mathrm{MeOTAD} / \mathrm{Au}\end{array}$ & 0.64 & 2.45 & 57 & 0.90 & [190] \\
\hline $\mathrm{Cs}_{2} \mathrm{NaBiI}_{6}$ & Spin coating & $\begin{array}{l}\mathrm{c}-\mathrm{TiO}_{2} / \mathrm{m}_{-}-\mathrm{TiO}_{2} / \text { perovskite/spiro- } \\
\mathrm{MeOTAD} / \mathrm{Au}\end{array}$ & 0.47 & 1.99 & 44 & 0.42 & [194] \\
\hline
\end{tabular}

In a subsequent work, Wu et al. used antisolvent dropping method and post-annealing process to realize high-quality $\mathrm{Cs}_{2} \mathrm{AgBiBr}_{6}$ films with ultra-smooth morphology, microsized grains, and high crystallinity (Fig. 16a). Then they fabricated inverted planar heterojunction solar cells with the as-prepared $\mathrm{Cs}_{2} \mathrm{AgBiBr}_{6}$ films and got a high PCE of $2.23 \%$ [189]. It should be noted that the $J-V$ hysteresis was clearly showed in the above-mentioned two works, which was closely related with the large amount of defects in $\mathrm{Cs}_{2} \mathrm{AgBiBr}_{6}$ films. Grancini et al. precisely controlled the perovskite deposition parameters to obtain a highly uniform and compact $\mathrm{Cs}_{2} \mathrm{AgBiBr}_{6}$ film, and modified the device interfaces with small molecular/polymeric hole-transporting materials to eliminate the hysteresis effect [190].

Recently, Liu et al. successfully prepared double perovskite $\mathrm{Cs}_{2} \mathrm{AgBiBr}_{6}$ thin films through a sequential vapor deposition procedure (Fig. 16b) [191]. The as-fabricated thin films with pure double perovskite phase showed large grain sizes, uniform and smooth surface morphology along with a PL lifetime of $117 \mathrm{~ns}$, indicative of a significant potential in photovoltaic applications. The solar cells with planar device structure obtained an optimal PCE of $1.37 \%$, which maintained $90 \%$ of the initial efficiency after $240 \mathrm{~h}$ storage under ambient condition (Fig. 16c-e). In addition to $\mathrm{Cs}_{2} \mathrm{AgBiBr}_{6}$, $\mathrm{Cs}_{2} \mathrm{NaBiI}_{6}$ has also been used as a light absorbing layer for PSCs and the device showed a good stability despite of a low efficiency of only $0.42 \%$ [192]. Relevant results for most types of double PSCs based on various device architectures are summarized in Table 3 [187-192].

At the initial design stage of lead-free halide double perovskite materials, researchers specifically emphasized the need to ensure the thermodynamic stability of the material [186-188]. Therefore, the halide double perovskite materials used in solar cells show excellent stability, which will be an outstanding advantage in practical application. In addition, the optical bandgap and other optoelectronic properties of the double perovskites can be optimized through the use of both B(I) and B(III) elements [186-194]. Thus, these characteristics make the halide double perovskite a good candidate for high-performance, stable and environmentally friendly solar cells.

\section{Conclusion and Prospect}

In this review, we focused our attention on environmentally friendly lead-free perovskite materials and combed the related progress on theoretical and experimental works. Herein, all of lead-free perovskites consisting of Sn (II), $\mathrm{Cu}$ (II), Bi(III), Sb (III), Sn (IV), Ti (IV), and Ag(I)Bi(III) were introduced from the isovalent and heterovalent elements replacement perspectives. We summarized the material preparation, device performance and stability of each lead-free perovskite materials and presented their possible issues and potential development prospects. For Sn-based 
perovskites, this type of material has a relatively high absorption coefficient (close to $1.80 \times 10^{4} \mathrm{~cm}^{-1}$ ), but the easy oxidation of $\mathrm{Sn}^{2+}$ to $\mathrm{Sn}^{4+}$ is also considered to be the main problem limiting the development of Sn-based PSCs. Similar to Sn-based perovskites, Ge-based perovskites also face the trouble of being easily decomposed by oxidation. In addition, compared with $\mathrm{Sn}^{2+}, \mathrm{Bi}$ and $\mathrm{Sb}$ are very stable when they are in the +3 valence state in the atmosphere. However, the efficiency of $\mathrm{Bi}$ - and Sb-based PSCs is still very low. Further optimization of their bandgaps as well as film morphology, manufacture methods and device structures are feasible solutions to further improve device performance. The transition metal perovskites represented by $\mathrm{Cu}$-based perovskites also need to reduce their wide optical bandgap and improve their charge transporting properties to develop more efficient PSCs. Besides, the application of $\mathrm{AB}(\mathrm{Ch}, \mathrm{X})_{3}$ perovskites for PSCs is facing great challenges due to the thermodynamic instability. In addition to the above-mentioned materials, the halide double perovskite has attracted people's attention because of its excellent stability and bandgap tunability.

In summary, we have reviewed the past experimental work and comprehensively understood the development and application prospects of current lead-free perovskite materials. Lead-free perovskites enable to avoid a high content of the toxic, polluting and bioaccumulative lead hazards and are more conducive to commercial production and application. At the same time, theoretical simulations have made important contributions to comprehending the fundamental physics behind the efficiency, stability, and carrier transport properties of lead-free PSCs. Using scientific theoretical calculations and reasonable solar cell preparation methods, we believe that the research on lead-free PSCs will soon have a clear breakthrough. Here, we are looking forward to the further development of lead-free perovskite materials and PSCs. Finally, we expect that the performance as well as the stability of lead-free perovskites, especially 2D layered perovskites, can be continuously improved to meet the needs of commercial development. In spite of slower development of lead-free perovskites than lead ones, we believe that the efficiency based on lead-free perovskite materials can break through $15 \%$ after further in-depth study, and we should keep warm toward this research.

Acknowledgements The authors acknowledge financial support from the National Key Research and Development Program of
China (Grant No. 2017YFB0404501), the National Major Fundamental Research Program of China (Grant No. 91833306), the National Natural Science Foundation of China (Grant Nos. 62074083, 62005131, 61705111 and 61704091), the Science Fund for Distinguished Young Scholars of Jiangsu Province of China (Grant No. BK20160039), the Natural Science Foundation of Jiangsu Province (Grant Nos. BM2012010 and BK20170899), the Priority Academic Program Development of Jiangsu Higher Education Institutions (Grant No. YX030003), the Jiangsu National Synergetic Innovation Center for Advanced Materials, the Synergetic Innovation Center for Organic Electronics and Information Displays, the National Postdoctoral Program for Innovative Talents (Grant No. BX201700122), and the Open Foundation from Jilin University (Grant Nos. IOSKL2017KF04 and IOSKL2018KF01).

Open Access This article is licensed under a Creative Commons Attribution 4.0 International License, which permits use, sharing, adaptation, distribution and reproduction in any medium or format, as long as you give appropriate credit to the original author(s) and the source, provide a link to the Creative Commons licence, and indicate if changes were made. The images or other third party material in this article are included in the article's Creative Commons licence, unless indicated otherwise in a credit line to the material. If material is not included in the article's Creative Commons licence and your intended use is not permitted by statutory regulation or exceeds the permitted use, you will need to obtain permission directly from the copyright holder. To view a copy of this licence, visit http://creativecommons.org/licenses/by/4.0/.

\section{References}

1. A. Kojima, K. Teshima, Y. Shirai, T. Miyasaka, Organometal halide perovskites as visible-light sensitizers for photovoltaic cells. J. Am. Chem. Soc. 131(17), 6050-6051 (2009). https:// doi.org/10.1021/ja809598r

2. M.M. Lee, J. Teuscher, T. Miyasaka, T.N. Murakami, H.J. Snaith, Efficient hybrid solar cells based on meso-superstructured organometal halide perovskites. Science 338(6107), 643-647 (2012). https://doi.org/10.1126/science.1228604

3. G. Xing, N. Mathews, S. Sun, S.S. Lim, Y.M. Lam et al., Long-range balanced electron- and hole-transport lengths in organic-inorganic $\mathrm{CH}_{3} \mathrm{NH}_{3} \mathrm{PbI}_{3}$. Science 342(6156), 344-347 (2013). https://doi.org/10.1126/science.1243167

4. S.D. Stranks, G.E. Eperon, G. Grancini, C. Menelaou, M.J.P. Alcocer et al., Electron-hole diffusion lengths exceeding 1 micrometer in an organometal trihalide perovskite absorber. Science 342(6156), 341-344 (2013). https://doi.org/10.1126/ science. 1243982

5. P. Docampo, J.M. Ball, M. Darwich, G.E. Eperon, H.J. Snaith, Efficient organometal trihalide perovskite planarheterojunction solar cells on flexible polymer substrates. Nat. Commun. 4, 2761-2766 (2013). https://doi.org/10.1038/ ncomms 3761

6. J.H. Heo, S.H. Im, J.H. Noh, T.N. Mandal, C.-S. Lim et al., Efficient inorganic-organic hybrid heterojunction solar 
cells containing perovskite compound and polymeric hole conductors. Nat. Photon. 7(6), 486-491 (2013). https://doi. org/10.1038/nphoton.2013.80

7. J. Burschka, N. Pellet, S.-J. Moon, R. Humphry-Baker, P. Gao et al., Sequential deposition as a route to high-performance perovskite-sensitized solar cells. Nature 499(7458), 316-319 (2013). https://doi.org/10.1038/nature12340

8. P. Gao, M. Grätzel, M.K. Nazeeruddin, Organohalide lead perovskites for photovoltaic applications. Energy Environ. Sci. 7(8), 2448-2463 (2014). https://doi.org/10.1039/c4ee0 0942h

9. O. Malinkiewicz, A. Yella, Y.H. Lee, G.M. Espallargas, M. Graetzel et al., Perovskite solar cells employing organic charge-transport layers. Nat. Photon. 8(2), 128-132 (2013). https://doi.org/10.1038/nphoton.2013.341

10. W. Chen, Y. Wu, Y. Yue, J. Liu, W. Zhang et al., Efficient and stable large-area perovskite solar cells with inorganic charge extraction layers. Science 350(6263), 944-948 (2015). https ://doi.org/10.1126/science.aad1015

11. M. Sessolo, H.J. Bolink, Solar cells@ Perovskite solar cells join the major league. Science 350(6263), 917 (2015). https ://doi.org/10.1126/science.aad5891

12. J. Xu, A. Buin, A.H. Ip, W. Li, O. Voznyy et al., Perovskite-fullerene hybrid materials suppress hysteresis in planar diodes. Nat. Commun. 6, 7081 (2015). https://doi. org/10.1038/ncomms8081

13. M.A. Green, K. Emery, Y. Hishikawa, W. Warta, E.D. Dunlop, Solar cell efficiency tables (version 46). Prog. Photovolt: Res. Appl. 23(7), 805-812 (2015). https://doi.org/10.1002/ pip. 2637

14. Z. Xiao, Q. Dong, C. Bi, Y. Shao, Y. Yuan et al., Solvent annealing of perovskite-induced crystal growth for photovoltaic-device efficiency enhancement. Adv. Mater. 26(37), 6503-6509 (2014). https://doi.org/10.1002/adma.201401685

15. A. Mei, X. Li, L. Liu, Z. Ku, T. Liu et al., A hole-conductorfree, fully printable mesoscopic perovskite solar cell with high stability. Science 345(6194), 295-298 (2014). https:// doi.org/10.1126/science. 1254763

16. D. Liu, T.L. Kelly, Perovskite solar cells with a planar heterojunction structure prepared using room-temperature solution processing techniques. Nat. Photon. 8(2), 133-138 (2013). https://doi.org/10.1038/nphoton.2013.342

17. C. Bi, Q. Wang, Y. Shao, Y. Yuan, Z. Xiao et al., Non-wetting surface-driven high-aspect-ratio crystalline grain growth for efficient hybrid perovskite solar cells. Nat. Commun. 6, 7747 (2015). https://doi.org/10.1038/ncomms8747

18. D.P. McMeekin, G. Sadoughi, W. Rehman, G.E. Eperon, M. Saliba et al., A mixed-cation lead mixed-halide perovskite absorber for tandem solar cells. Science 351(6269), 151-155 (2016). https://doi.org/10.1126/science.aad5845

19. Y. Chen, T. Chen, L. Dai, Layer-by-layer growth of $\mathrm{CH}_{3} \mathrm{NH}_{3} \mathrm{PbI}_{(3-\mathrm{x})} \mathrm{Cl}_{\mathrm{x}}$ for highly efficient planar heterojunction perovskite solar cells. Adv. Mater. 27(6), 1053-1059 (2015). https://doi.org/10.1002/adma.201404147
20. W.S. Yang, J.H. Noh, N.J. Jeon, Y.C. Kim, S. Ryu et al., High-performance photovoltaic perovskite layers fabricated through intramolecular exchange. Science 348(6420), 1234 1237 (2015). https://doi.org/10.1126/science.aaa9272

21. J. Wang, Y. Liu, X. Chen, C. Chen, P. Chen et al., Functional metal oxides in perovskite solar cells. ChemPhysChem 20(20), 2580-2586 (2019). https://doi.org/10.1002/ cphc. 201900447

22. D. Bi, W. Tress, M.I. Dar, P. Gao, J. Luo et al., Efficient luminescent solar cells based on tailored mixed-cation perovskites. Sci. Adv. 2(1), e1501170 (2016). https://doi. org/10.1126/sciadv.1501170

23. K. Meng, S. Gao, L. Wu, G. Wang, X. Liu et al., Two-dimensional organic-inorganic hybrid perovskite photonic films. Nano Lett. 16(7), 4166-4173 (2016). https://doi.org/10.1021/ acs.nanolett.6b01046

24. N.J. Jeon, H. Na, E.H. Jung, T.-Y. Yang, Y.G. Lee et al., A fluorene-terminated hole-transporting material for highly efficient and stable perovskite solar cells. Nat. Energy 3(8), 682-689 (2018). https://doi.org/10.1038/s41560-018-0200-6

25. B. Hailegnaw, S. Kirmayer, E. Edri, G. Hodes, D. Cahen, Rain on methylammonium lead iodide based perovskites: Possible environmental effects of perovskite solar cells. J. Phys. Chem. Lett. 6(9), 1543-1547 (2015). https://doi. org/10.1021/acs.jpclett.5b00504

26. R. Wang, J. Wang, S. Tan, Y. Duan, Z.-K. Wang et al., Opportunities and challenges of lead-free perovskite optoelectronic devices. Trends in Chem. 1(4), 368-379 (2019). https://doi. org/10.1016/j.trechm.2019.04.004

27. F. Giustino, H.J. Snaith, Toward lead-free perovskite solar cells. ACS Energy Lett. 1(6), 1233-1240 (2016). https://doi. org/10.1021/acsenergylett.6b00499

28. Z. Shi, J. Guo, Y. Chen, Q. Li, Y. Pan et al., Lead-free organic-inorganic hybrid perovskites for photovoltaic applications: recent advances and perspectives. Adv. Mater. 29(16), 1605005 (2017). https://doi.org/10.1002/adma.201605005

29. S. Yang, W. Fu, Z. Zhang, H. Chen, C.-Z. Li, Recent advances in perovskite solar cells: efficiency, stability and lead-free perovskite. J. Mater. Chem. A 5(23), 11462-11482 (2017). https://doi.org/10.1039/c7ta00366h

30. P. Xu, S. Chen, H.-J. Xiang, X.-G. Gong, S.-H. Wei, Influence of defects and synthesis conditions on the photovoltaic performance of perovskite semiconductor $\mathrm{CsSnI}_{3}$. Chem. Mater. 26(2), 6068-6073 (2014). https://doi.org/10.1021/cm503122j

31. S.F. Hoefler, G. Trimmel, T. Rath, Progress on lead-free metal halide perovskites for photovoltaic applications: a review. Monatsh. Chem. 148(5), 795-826 (2017). https:// doi.org/10.1007/s00706-017-1933-9

32. M. Lyu, J.-H. Yun, P. Chen, M. Hao, L. Wang, Addressing toxicity of lead: progress and applications of low-toxic metal halide perovskites and their derivatives. Adv. Energy Mater. 7(15), 1602512-1602537 (2017). https://doi.org/10.1002/ aenm.201602512

33. W. Ming, H. Shi, M.-H. Du, Large dielectric constant, high acceptor density, and deep electron traps in perovskite solar 
cell material CsGeI ${ }_{3}$. J. Mater. Chem. A 4(13), 13852-13875 (2016). https://doi.org/10.1039/C6TA04685A

34. F. Hong, B. Saparov, W. Meng, Z. Xiao, D.B. Mitzi et al., Viability of lead-free perovskites with mixed chalcogen and halogen anions for photovoltaic applications. J. Phys. Chem. C 120(12), 6435-6441 (2016). https://doi.org/10.1021/acs. jpcc.6b00920

35. G.E. Eperon, S.D. Stranks, C. Menelaou, M.B. Johnston, L.M. Herz et al., Formamidinium lead trihalide: a broadly tunable perovskite for efficient planar heterojunction solar cells. Energy Environ. Sci. 7(3), 982-989 (2014). https://doi. org/10.1039/c3ee43822h

36. P. Sun, Q. Li, L. Yang, Z. Li, Theoretical insights into a potential lead-free hybrid perovskite: substituting $\mathrm{Pb}^{2+}$ with $\mathrm{Ge}^{2+}$. Nanoscale 8(3), 1503-1513 (2016). https://doi. org/10.1039/c5nr05337d

37. Y. Zhao, K. Zhu, Organic-inorganic hybrid lead halide perovskites for optoelectronic and electronic applications. Chem. Soc. Rev. 45(3), 655-689 (2016). https://doi.org/10.1039/ $\mathrm{c} 4 \mathrm{cs} 00458 \mathrm{~b}$

38. N. Pellet, P. Gao, G. Gregori, T.-Y. Yang, M.K. Nazeeruddin et al., Mixed-organic-cation perovskite photovoltaics for enhanced solar-light harvesting. Angew. Chem. Int. Ed. 53(12), 3151-3157 (2014). https://doi.org/10.1002/ anie. 201309361

39. N.H. Tiep, Z. Ku, H.J. Fan, Recent advances in improving the stability of perovskite solar cells. Adv. Energy Mater. 6(3), 1501420-1501456 (2016). https://doi.org/10.1002/ aenm.201501420

40. Y. Zhang, J. Yin, M.R. Parida, G.H. Ahmed, J. Pan et al., Direct-indirect nature of the bandgap in lead-free perovskite nanocrystals. J. Phys. Chem. Lett. 8(1), 3173-3188 (2017). https://doi.org/10.1021/acs.jpclett.7b01381

41. N.J. Jeon, J.H. Noh, W.S. Yang, Y.C. Kim, S. Ryu et al., Compositional engineering of perovskite materials for highperformance solar cells. Nature 517(7535), 476-480 (2015). https://doi.org/10.1038/nature14133

42. D.E. Starr, G. Sadoughi, E. Handick, R.G. Wilks, J.H. Alsmeier et al., Direct observation of an inhomogeneous chlorine distribution in $\mathrm{CH}_{3} \mathrm{NH}_{3} \mathrm{PbI}_{3-\mathrm{x}} \mathrm{Cl}_{\mathrm{x}}$ layers: surface depletion and interface enrichment. Energy Environ. Sci. 8(5), 1609-1615 (2015). https://doi.org/10.1039/c5ee00403a

43. S. Dharani, H.A. Dewib, R.R. Prabhakarb, T. Baikieb, C. Shic et al., Incorporation of $\mathrm{Cl}$ in sequentially deposited lead halide perovskite films for highly efficient mesoporous solar cells. Nanoscale 6(22), 13854-13860 (2014). https://doi. org/10.1039/C4NR04007D

44. W. Zhang, M. Saliba, D.T. Moore, S.K. Pathak, M.T. Hörantner et al., Ultrasmooth organic-inorganic perovskite thin-film formation and crystallization for efficient planar heterojunction solar cells. Nat. Commun. 6, 6142-6151 (2015). https:// doi.org/10.1038/ncomms7142

45. J. Qing, H.-T. Chandran, Y.-H. Cheng, X.-K. Liu, H.-W. Li et al., Chlorine incorporation for enhanced performance of planar perovskite solar cell based on lead acetate precursor.
ACS Appl. Mater. Interfaces. 7(41), 23110-23116 (2015). https://doi.org/10.1021/acsami.5b06819

46. X. Lu, Y. Wang, C.C. Stoumpos, Q. Hu, X. Guo et al., Enhanced structural stability and photo responsiveness of $\mathrm{CH}_{3} \mathrm{NH}_{3} \mathrm{SnI}_{3}$ perovskite via pressure-induced amorphization and recrystallization. Adv. Mater. 28(39), 8663-8668 (2016). https://doi.org/10.1002/adma.201600771

47. M. Jiang, J. Wu, F. Lan, Q. Tao, D. Gao et al., Enhancing the performance of planar organo-lead halide perovskite solar cells by using a mixed halide source. J. Mater. Chem. A 3(3), 963-967 (2015). https://doi.org/10.1039/c4ta05373g

48. L. Huang, Z. Hu, G. Yue, J. Liu, X. Cui et al., $\mathrm{CH}_{3} \mathrm{NH}_{3} \mathrm{PbI}_{3-\mathrm{x}} \mathrm{Cl}_{\mathrm{x}}$ films with coverage approaching $100 \%$ and with highly oriented crystal domains for reproducible and efficient planar heterojunction perovskite solar cells. Phys. Chem. Chem. Phys. 17(34), 22015-22022 (2015). https://doi. org/10.1039/c5cp03934g

49. T.G. Kim, S.W. Seo, H. Kwon, J. Hahn, J.W. Kim, Influence of halide precursor type and its composition on the electronic properties of vacuum deposited perovskite films. Phys. Chem. Chem. Phys. 17(37), 24342-24348 (2015). https ://doi.org/10.1039/c5cp04207k

50. J. Albero, A.M. Asiri, H. Garcia, Influence of the composition of hybrid perovskites on their performance in solar cells. J. Mater. Chem. A 4(12), 4353-4364 (2016). https:// doi.org/10.1039/c6ta00334f

51. B. Ghosh, S. Chakraborty, H. Wei, C. Guet, S. Li et al., Poor photovoltaic performance of $\mathrm{Cs}_{3} \mathrm{Bi}_{2} \mathrm{I}_{9}$ : an insight through first-principles calculations. J. Phys. Chem. C 121(32), 17062-17081 (2017). https://doi.org/10.1021/acs.jpcc.7b035 01

52. Y. Sun, J. Shi, J. Lian, W. Gao, M.L. Agiorgousis et al., Discovering lead-free perovskite solar materials with a splitanion approach. Nanoscale 8(12), 6284-6290 (2016). https ://doi.org/10.1039/c5nr04310g

53. G.E. Eperon, C.E. Beck, H.J. Snaith, Cation exchange for thin film lead iodide perovskite interconversion. Mater. Horiz. 3(1), 63-71 (2016). https://doi.org/10.1039/c5mh00170f

54. J.H. Heo, S.H. Im, Highly reproducible, efficient hysteresisless $\mathrm{CH}_{3} \mathrm{NH}_{3} \mathrm{PbI}_{3-\mathrm{x}} \mathrm{Cl}_{\mathrm{x}}$ planar hybrid solar cells without requiring heat-treatment. Nanoscale 8(5), 2554-2560 (2016). https://doi.org/10.1039/c5nr08458j

55. T.M. Brenner, D.A. Egger, L. Kronik, G. Hodes, D. Cahen, Hybrid organic-inorganic perovskites: low-cost semiconductors with intriguing charge-transport properties. Nat. Rev. Mater. 1, 15007-15023 (2015). https://doi.org/10.1038/natre vmats. 2015.7

56. N.K. Noel, S.D. Stranks, A. Abate, C. Wehrenfennig, S. Guarnera et al., Lead-free organic-inorganic tin halide perovskites for photovoltaic applications. Energy Environ. Sci. 7(9), 3061-3068 (2014). https://doi.org/10.1039/c4ee01076k

57. N. Leblanc, N. Mercier, L. Zorina, S. Simonov, P. AubanSenzier et al., Large spontaneous polarization and clear hysteresis loop of a room-temperature hybrid ferroelectric based on mixed-halide $\mathrm{BiI}_{3} \mathrm{Cl}_{2}$ polar chains and methylviologen 
dication. J. Am. Chem. Soc. 133(38), 14924-14927 (2011). https://doi.org/10.1021/ja206171s

58. W. Bi, N. Leblanc, N. Mercier, P. Auban-Senzier, C. Pasquier, Thermally induced $\mathrm{Bi}$ (III) lone pair stereoactivity: ferroelectric phase transition and semiconducting properties of (MV)BiBr 5(MV = methylviologen). Chem. Mater. 21(18), 4099-4101 (2009). https://doi.org/10.1021/cm9016003

59. B.-W. Park, B. Philippe, X. Zhang, H. Rensmo, G. Boschloo et al., Bismuth based hybrid perovskites $\mathrm{A}_{3} \mathrm{Bi}_{2} \mathrm{I}_{9}$ (a: methylammonium or cesium) for solar cell application. Adv. Mater. 27(43), 6806-6813 (2015). https://doi.org/10.1002/ adma. 201501978

60. T. Krishnamoorthy, H. Ding, C. Yan, W.L. Leong, T. Baikie et al., Lead-free germanium iodide perovskite materials for photovoltaic applications. J. Mater. Chem. A 3(47), 23829 23832 (2015). https://doi.org/10.1039/c5ta05741h

61. C.C. Stoumpos, L. Frazer, D.J. Clark, Y.S. Kim, S.H. Rhim et al., Hybrid germanium iodide perovskite semiconductors: active lone pairs, structural distortions, direct and indirect energy gaps, and strong nonlinear optical properties. J. Am. Chem. Soc. 137(21), 6804-6819 (2015). https://doi. org/10.1021/jacs.5b01025

62. B. Saparov, F. Hong, J.-P. Sun, H.-S. Duan, W. Meng et al., Thin-film preparation and characterization of $\mathrm{Cs}_{3} \mathrm{Sb}_{2} \mathrm{I}_{9}$ : a lead-free layered perovskite semiconductor. Chem. Mater. 27(16), 5622-5632 (2015). https://doi.org/10.1021/acs. chemmater.5b01989

63. D. Cortecchia, H.A. Dewi, J. Yin, A. Bruno, S. Chen et al., Lead-free MA2 CuClxBr 4-x hybrid perovskites. Inorg. Chem. 55(3), 1044-1052 (2016). https://doi.org/10.1021/ acs.inorgchem.5b01896

64. G. Giorgi, J.-I. Fujisawa, H. Segawa, K. Yamashita, Small photocarrier effective masses featuring ambipolar transport in methylammonium lead iodide perovskite: a density functional analysis. J. Phys. Chem. Lett. 4(24), 4213-4216 (2013). https ://doi.org/10.1021/jz4023865

65. J. Feng, B. Xiao, Crystal structures, optical properties, and effective mass tensors of $\mathrm{CH}_{3} \mathrm{NH}_{3} \mathrm{PbX}_{3}(\mathrm{X}=\mathrm{I}$ and $\mathrm{Br})$ phases predicted from HSE06. J. Phys. Chem. Lett. 5(7), 1278-1282 (2014). https://doi.org/10.1021/jz500480m

66. M.R. Filip, F. Giustino, Computational screening of homovalent lead substitution in organic-inorganic halide perovskites. J. Phys. Chem. C 120(1), 166-173 (2015). https://doi. org/10.1021/acs.jpcc.5b11845

67. Z. Xiao, Z. Song, Y. Yan, From lead halide perovskites to lead-free metal halide perovskites and perovskite derivatives. Adv. Mater. 31(47), 1803792-1803813 (2019). https://doi. org/10.1002/adma.201803792

68. P. Umari, E. Mosconi, F. De Angelis, Relativistic GW calculations on $\mathrm{CH}_{3} \mathrm{NH}_{3} \mathrm{PbI}_{3}$ and $\mathrm{CH}_{3} \mathrm{NH}_{3} \mathrm{SnI}_{3}$ perovskites for solar cell applications. Sci. Rep. 4, 4467-4473 (2014). https ://doi.org/10.1038/srep04467

69. F. Li, C. Zhang, J.H. Huang, H. Fan, H. Wang et al., A cationexchange approach for the fabrication of efficient methylammonium tin iodide perovskite solar cells. Angew. Chem.
Int. Ed. 58(20), 6688-6692 (2019). https://doi.org/10.1002/ anie. 201902418

70. F. Hao, C.C. Stoumpos, D.H. Cao, R.P.H. Chang, M.G. Kanatzidis, Lead-free solid-state organic-inorganic halide perovskite solar cells. Nat. Photon. 8(6), 489-494 (2014). https://doi.org/10.1038/nphoton.2014.82

71. M.H. Kumar, S. Dharani, W.L. Leong, P.P. Boix, R.R. Prabhakar et al., Lead-free halide perovskite solar cells with high photocurrents realized through vacancy modulation. Adv. Mater. 26(41), 7122-7127 (2014). https://doi.org/10.1002/ adma.201401991

72. J.H. Noh, S.H. Im, J.H. Heo, T.N. Mandal, S.I. Seok, Chemical management for colorful, efficient, and stable inorganicorganic hybrid nanostructured solar cells. Nano Lett. 13(4), 1764-1769 (2013). https://doi.org/10.1021/nl400349b

73. T. Yokoyama, D.H. Cao, C.C. Stoumpos, T.B. Song, Y. Sato et al., Overcoming short-circuit in lead-free $\mathrm{CH}_{3} \mathrm{NH}_{3} \mathrm{SnI}_{3}$ perovskite solar cells via kinetically controlled gas-solid reaction film fabrication process. J. Phys. Chem. Lett. 7(5), 776-782 (2016). https://doi.org/10.1021/acs.jpclett.6b00118

74. F. Hao, C.C. Stoumpos, P. Guo, N. Zhou, T.J. Marks et al., Solvent-mediated crystallization of $\mathrm{CH}_{3} \mathrm{NH}_{3} \mathrm{SnI}_{3}$ films for heterojunction depleted perovskite solar cells. J. Am. Chem. Soc. 137(35), 11445-11452 (2015). https://doi.org/10.1021/ jacs.5b06658

75. M. Weiss, J. Horn, C. Richter, D. Schlettwein, Preparation and characterization of methylammonium tin iodide layers as photovoltaic absorbers. Phys. Status Solidi A 213(4), 975-981 (2016). https://doi.org/10.1002/pssa.201532594

76. S. Chen, A. Walsh, X.G. Gong, S.-H. Wei, Classification of lattice defects in the kesterite $\mathrm{Cu}_{2} \mathrm{ZnSnS}_{4}$ and $\mathrm{Cu}_{2} \mathrm{ZnSnSe}_{4}$ earth-abundant solar cell absorbers. Adv. Mater. 25(11), 1522-1539 (2013). https://doi.org/10.1002/adma.201203146

77. M.C. Jung, S.R. Raga, Y. Qi, Properties and solar cell applications of $\mathrm{Pb}$-free perovskite films formed by vapor deposition. RSC Adv. 6(4), 2819-2825 (2016). https://doi.org/10.1039/ c5ra21291j

78. D.E. Scaife, P.F. Weller, W.G. Fisher, Crystal preparation and properties of cesium tin(ii) trihalides. J. Solid State Chem. 9(3), 308-314 (1974). https://doi.org/10.1016/00224596(74)90088-7

79. Z. Chen, J.J. Wang, Y. Ren, C. Yu, K. Shum, Schottky solar cells based on $\mathrm{CsSnI}_{3}$ thin-films. Appl. Phys. Lett. 101(9), 093901-093904 (2012). https://doi.org/10.1063/1.4748888

80. D. Sabba, H.K. Mulmudi, R.R. Prabhakar, T. Krishnamoorthy, T. Baikie et al., Impact of anionic Br- substitution on open circuit voltage in lead free perovskite $\left(\mathrm{CsSnI}_{3-\mathrm{x}} \mathrm{Br}_{\mathrm{x}}\right)$ solar cells. J. Phys. Chem. C 119(4), 1763-1767 (2015). https ://doi.org/10.1021/jp5126624

81. Y. Zhou, H.F. Garces, B.S. Senturk, A.L. Ortiz, N.P. Padture, Roomtemperature "one-pot" solution synthesis of nanoscale $\mathrm{CsSnI}_{3}$ orthorhombic perovskite thin films andparticles. Mater. Lett. 110, 127-129 (2013). https://doi.org/10.1016/j. matlet.2013.08.011

82. S. Dharani, H.K. Mulmudi, N. Yantara, P.T. Thu-Trang, N.G. Park et al., High efficiency electrospun $\mathrm{TiO}_{2}$ nanofiber based 
hybrid organic-inorganic perovskite solar cell. Nanoscale 6(3), 1675-1679 (2014). https://doi.org/10.1039/c3nr04857h

83. K. Shum, Z. Chen, J. Qureshi, C. Yu, J.J. Wang et al., Synthesis and characterization of $\mathrm{CsSNi}_{3}$ thin films. Appl. Phys. Lett. 96(22), 221903 (2010). https://doi.org/10.1063/1.34425 11

84. I. Chung, J.H. Song, J. Im, J. Androulakis, C.D. Malliakas et al., $\mathrm{CsSnI}_{3}$ : semiconductor or metal? High electrical conductivity and strong near-infrared photoluminescence from a single material. High hole mobility and phase-transitions. J. Am. Chem. Soc. 134(20), 8579-8587 (2012). https://doi. org/10.1021/ja301539s

85. K.P. Marshall, R.I. Walton, R.A. Hatton, Tin perovskite/ fullerene planar layer photovoltaics: improving the efficiency and stability of lead-free devices. J. Mater. Chem. A 3(21), 11631-11640 (2015). https://doi.org/10.1039/c5ta02950c

86. L. Liang, P. Gao, Lead-free hybrid perovskite absorbers for viable application: can we eat the cake and have it too? Adv. Sci. 5(2), 1700331-1700364 (2018). https://doi.org/10.1002/ advs. 201700331

87. S.J. Lee, S.S. Shin, Y.C. Kim, D. Kim, T.K. Ahn et al., Fabrication of efficient formamidinium tin iodide perovskite solar cells through $\mathrm{SnF}_{2}$-pyrazine complex. J. Am. Chem. Soc. 138(12), 3974-3977 (2016). https://doi.org/10.1021/ jacs.6b00142

88. Z. Zhao, F. Gu, Y. Li, W. Sun, S. Ye et al., Mixed-organiccation tin iodide for lead-free perovskite solar cells with an efficiency of 8.12. Adv. Sci. 4(11), 1700204-1700210 (2017). https://doi.org/10.1002/advs.201700204

89. W. Ke, C.C. Stoumpos, M. Zhu, L. Mao, I. Spanopoulos et al., Enhanced photovoltaic performance and stability with a new type of hollow 3D perovskite \{en\}FASnI 3 . Sci. Adv. 3(8), e1701293-e1701301 (2017). https://doi.org/10.1126/sciad v. 1701293

90. K.P. Marshall, M. Walker, R.I. Walton, R.A. Hatton, Enhanced stability and efficiency in hole-transport-layer-free $\mathrm{CsSnI}_{3}$ perovskite photovoltaics. Nat. Energy 1(12), 1617816186 (2016). https://doi.org/10.1038/nenergy.2016.178

91. M. Zhang, M. Lyu, J.H. Yun, M. Noori, X. Zhou et al., Low-temperature processed solar cells with formamidinium tin halide perovskite/fullerene heterojunctions. Nano Res. 9(6), 1570-1577 (2016). https://doi.org/10.1007/s 1227 4-016-1051-8

92. R.L. Milot, G.E. Eperon, T. Green, H.J. Snaith, M.B. Johnston et al., Radiative monomolecular recombination boosts amplified spontaneous emission in $\mathrm{HC}\left(\mathrm{NH}_{2}\right)_{2} \mathrm{SnI}_{3}$ perovskite films. J. Phys. Chem. Lett. 7(20), 4178-4184 (2016). https:// doi.org/10.1021/acs.jpclett.6b02030

93. Y. Dang, Y. Zhou, X. Liu, D. Ju, S. Xia, H. Xia, X. Tao, Formation of hybrid perovskite tin iodide single crystals by top-seeded solution growth. Angew. Chem. Int. Ed. 55(10), 3447-3450 (2016). https://doi.org/10.1002/anie.201511792

94. D.B. Mitzi, K. Liang, Synthesis, resistivity, and thermal properties of the cubic perovskite $\mathrm{NH}_{2} \mathrm{CH}_{5} \mathrm{NH}_{2} \mathrm{SnI}_{3}$ and related systems. J. Solid State Chem. 134(2), 376-381 (1997). https ://doi.org/10.1006/jssc.1997.7593
95. Z. Zhu, C.C. Chueh, N. Li, C. Mao, A.K.-Y. Jen, Realizing efficient lead-free formamidinium tin triiodide perovskite solar cells via a sequential deposition route. Adv. Mater. 30(6), 1703800-1703808 (2018). https://doi.org/10.1002/ adma.201703800

96. W. Liao, D. Zhao, Y. Yu, C.R. Grice, C. Wang et al., Leadfree inverted planar formamidinium tin triiodide perovskite solar cells achieving power conversion efficiencies up to 622. Adv. Mater. 28(42), 9333-9340 (2016). https://doi. org/10.1002/adma.201602992

97. W. Ke, C.C. Stoumpos, J.L. Logsdon, M.R. Wasielewski, Y. Yan et al., $\mathrm{Tio}_{2}$-zns cascade electron transport layer for efficient formamidinium tin iodide perovskite solar cells. J. Am. Chem. Soc. 138(45), 14998-15003 (2016). https://doi. org/10.1021/jacs.6b08790

98. F. Wang, J. Ma, F. Xie, L. Li, J. Chen et al., Organic cationdependent degradation mechanism of organotin halide perovskites. Adv. Funct. Mater. 26(20), 3417-3423 (2016). https ://doi.org/10.1002/adfm.201505127

99. T.M. Koh, T. Krishnamoorthy, N. Yantara, C. Shi, W.L. Leong et al., Formamidinium tin-based perovskite with low Eg for photovoltaic applications. J. Mater. Chem. A 3(29), 14996-15000 (2015). https://doi.org/10.1039/c5ta00190k

100. F. Gu, S. Ye, Z. Zhao, H. Rao, Z. Liu et al., Improving performance of lead-free formamidinium tin triiodide perovskite solar cells by tin source purification. Sol. RRL 2(10), 1870217 (2018). https://doi.org/10.1002/solr.201870217

101. Z. Lin, C. Liu, G. Liu, J. Yang, X. Duan et al., Efficient inverted tin-based perovskite solar cells via bidentate coordination effect of 8-Hydroxyquinoline. Chem. Commun. 56(28), 4007-4010 (2020). https://doi.org/10.1039/d0cc0 $1106 \mathrm{a}$

102. J. Cao, Q. Tai, P. You, G. Tang, T. Wang et al., Enhanced performance of tin-based perovskite solar cells induced by an ammonium hypophosphite additive. J. Mater. Chem. A 7(46), 26580-26585 (2019). https://doi.org/10.1039/c9ta08679j

103. T. Wu, X. Liu, X. He, Y. Wang, X. Meng et al., Efficient and stable tin-based perovskite solar cells by introducing $\pi$-conjugated lewis base. Sci. China Chem. 63(1), 107-115 (2019). https://doi.org/10.1007/s11426-019-9653-8

104. X. Meng, T. Wu, X. Liu, X. He, T. Noda et al., Highly reproducible and efficient $\mathrm{FASnI}_{3}$ perovskite solar cells fabricated with volatilizable reducing solvent. J. Phys. Chem. Lett. 11(8), 2965-2971 (2020). https://doi.org/10.1021/acs.jpcle tt.0c00923

105. M. Saliba, T. Matsui, K. Domanski, J.-Y. Seo, A. Ummadisingu et al., Incorporation of rubidium cations into perovskite solar cells improves photovoltaic performance. Science 354(6309), 206-209 (2016). https://doi.org/10.1126/scien ce.aah5557

106. N.J. Jeon, J.H. Noh, Y.C. Kim, W.S. Yang, S. Ryu et al., Solvent engineering for high-performance inorganic-organic hybrid perovskite solar cells. Nat. Mater. 13(9), 897-903 (2014). https://doi.org/10.1038/nmat4014

107. N. Ahn, D.-Y. Son, I.-H. Jang, S.M. Kang, M. Choi et al., Highly reproducible perovskite solar cells with average 
efficiency of $18.3 \%$ and best efficiency of $19.7 \%$ fabricated via lewis base adduct of lead(ii) iodide. J. Am. Chem. Soc. 137(27), 8696-8699 (2015). https://doi.org/10.1021/ jacs.5b04930

108. W. Ke, C.C. Stoumpos, I. Spanopoulos, L. Mao, M. Chen et al., Efficient lead-free solar cells based on hollow $\{\mathrm{en}\}$ $\mathrm{MASnI}_{3}$ perovskites. J. Am. Chem. Soc. 139(41), 1480014806 (2017). https://doi.org/10.1021/jacs.7b09018

109. W. Ke, C.C. Stoumpos, I. Spanopoulos, M. Chen, M.R. Wasielewski et al., Diammonium cations in the $\mathrm{FASnI}_{3}$ perovskite structure lead to lower dark currents and more efficient solar cells. ACS Energy Lett. 3(7), 1470-1476 (2018). https://doi.org/10.1021/acsenergylett.8b00687

110. X. Li, D. Bi, C. Yi, J.D. Décoppet, J. Luo et al., A vacuum flash-assisted solution process for high-efficiency large-area perovskite solar cells. Science 353, 58-62 (2016). https://doi. org/10.1126/science.aaf8060

111. F. Xu, T. Zhang, G. Li, Y. Zhao, Mixed cation hybrid lead halide perovskites with enhanced performance and stability. J. Mater. Chem. A 5(23), 11450-11461 (2017). https://doi. org/10.1039/c7ta00042a

112. H. Tan, A. Jain, O. Voznyy, X. Lan, F.P.G. Arquer et al., Efficient and stable solution-processed planar perovskite solar cells via contact passivation. Science 355(6326), 722-726 (2017). https://doi.org/10.1126/science.aai9081

113. W.S. Yang, B.-W. Park, E.H. Jung, N.J. Jeon, Y.C. Kim et al., Iodide management in formamidinium-lead-halide-based perovskite layers for efficient solar cells. Science 356(6345), 1376-1379 (2017). https://doi.org/10.1126/science.aan2301

114. X. Liu, Z. Yang, C.C. Chueh, A. Rajagopal, S.T. Williams et al., Improved efficiency and stability of $\mathrm{Pb}$-Sn binary perovskite solar cells by cs substitution. J. Mater. Chem. A 4(46), 17939-17945 (2016). https://doi.org/10.1039/c6ta07712a

115. I.C. Smith, E.T. Hoke, D. Solis-Ibarra, M.D. McGehee, H.I. Karunadasa, A layered hybrid perovskite solar-cell absorber with enhanced moisture stability. Angew. Chem. Int. Ed. 53(42), 11232-11235 (2014). https://doi.org/10.1002/ anie. 201406466

116. W. Peng, J. Yin, K.T. Ho, O. Ouellette, M. De Bastiani et al., Ultralow self-doping in two-dimensional hybrid perovskite single crystals. Nano Lett. 17(8), 4759-4767 (2017). https:// doi.org/10.1021/acs.nanolett.7b01475

117. Y. Lin, Y. Bai, Y. Fang, Q. Wang, Y. Deng et al., Suppressed ion migration in low-dimensional perovskites. ACS Energy Lett. 2(7), 1571-1572 (2017). https://doi.org/10.1021/acsen ergylett.7b00442

118. C.-M. Tsai, Y.-P. Lin, M.K. Pola, S. Narra, E. Jokar et al., Control of crystal structures and optical properties with hybrid formamidinium and 2-hydroxyethylammonium cations for mesoscopic carbon-electrode tin-based perovskite solar cells. ACS Energy Lett. 3(9), 2077-2085 (2018). https ://doi.org/10.1021/acsenergylett.8b01046

119. E. Jokar, C.H. Chien, C.M. Tsai, A. Fathi, E.W. Diau, Robust tin-based perovskite solar cells with hybrid organic cations to attain efficiency approaching 10\%. Adv. Mater. 31(2), e1804835 (2019). https://doi.org/10.1002/adma.201804835
120. D.H. Cao, C.C. Stoumpos, T. Yokoyama, J.L. Logsdon, T.-B. Song et al., Thin films and solar cells based on semiconducting two-dimensional ruddlesden-popper $\left(\mathrm{CH}_{3}\left(\mathrm{CH}_{2}\right)_{3} \mathrm{NH}_{3}\right)_{2}\left(\mathrm{CH}_{3} \mathrm{NH}_{3}\right)_{\mathrm{n}-1} \mathrm{Sn}_{\mathrm{n}} \mathrm{I}_{3 \mathrm{n}+1}$ perovskites. ACS Energy Lett. 2(5), 982-990 (2017). https://doi.org/10.1021/ acsenergylett.7b00202

121. K. Yao, X. Wang, Y. Xu, F. Li, L. Zhou, Multilayered perovskite materials based on polymeric-ammonium cations for stable large-area solar cell. Chem. Mater. 28(9), 3131-3138 (2016). https://doi.org/10.1021/acs.chemmater.6b00711

122. C. Ma, C. Leng, Y. Ji, X. Wei, K. Sun et al., 2D/3D perovskite hybrids as moisture-tolerant and efficient light absorbers for solar cells. Nanoscale 8(43), 18309-18314 (2016). https:// doi.org/10.1039/c6nr04741f

123. F. Wang, X. Jiang, H. Chen, Y. Shang, H. Liu et al., 2D-quasi2D-3D hierarchy structure for tin perovskite solar cells with enhanced efficiency and stability. Joule 2(12), 2732-2743 (2018). https://doi.org/10.1016/j.joule.2018.09.012

124. X. Zhang, G. Wu, W. Fu, M. Qin, W. Yang et al., Orientation regulation of phenylethylammonium cation based 2D perovskite solar cell with efficiency higher than $11 \%$. Adv. Energy Mater. 8(14), 1702498 (2018). https://doi.org/10.1002/ aenm.201702498

125. A.Z. Chen, M. Shiu, J.H. Ma, M.R. Alpert, D. Zhang et al., Origin of vertical orientation in two-dimensional metal halide perovskites and its effect on photovoltaic performance. Nat. Commun. 9(1), 1336 (2018). https://doi.org/10.1038/s4146 7-018-03757-0

126. J. Qiu, Y. Xia, Y. Zheng, W. Hui, H. Gu et al., 2D intermediate suppression for efficient ruddlesden-popper (RP) phase lead-free perovskite solar cells. ACS Energy Lett. 4(7), 15131520 (2019). https://doi.org/10.1021/acsenergylett.9b00954

127. Y. Liao, H. Liu, W. Zhou, D. Yang, Y. Shang et al., Highly oriented low-dimensional tin halide perovskites with enhanced stability and photovoltaic performance. J. Am. Chem. Soc. 139(19), 6693-6699 (2017). https://doi. org/10.1021/jacs.7b01815

128. H. Xu, Y. Jiang, T. He, S. Li, H. Wang et al., Orientation regulation of tin-based reduced-dimensional perovskites for highly efficient and stable photovoltaics. Adv. Funct. Mater. 29(47), 1807696 (2019). https://doi.org/10.1002/adfm.20180 7696

129. J. Wang, H. Shen, W. Li, S. Wang, J. Li et al., The role of chloride incorporation in lead-free $2 \mathrm{D}$ perovskite $(\mathrm{BA})_{2} \mathrm{SnI}_{4}$ : morphology, photoluminescence, phase transition, and charge transport. Adv. Sci. 6(5), 1802019 (2019). https://doi. org/10.1002/advs.201802019

130. M. Chen, M.G. Ju, M.Y. Hu, Z.H. Dai, Y. Hu et al., Lead-free dion-jacobson tin halide perovskites for photovoltaics. ACS Energy Lett. 4(1), 276-277 (2019). https://doi.org/10.1021/ acsenergylett.8b02051

131. B.E. Cohen, Y. Li, Q. Meng, L. Etgar, Dion-jacobson twodimensional perovskite solar cells based on benzene dimethanammonium cation. Nano Lett. 19(4), 2588-2597 (2019). https://doi.org/10.1021/acs.nanolett.9b00387 
132. C. Ma, D. Shen, T.W. Ng, M.F. Lo, C.S. Lee, 2D perovskites with short interlayer distance for high-performance solar cell application. Adv. Mater. 30(22), 1800710-1800715 (2018). https://doi.org/10.1002/adma.201800710

133. C.C. Stoumpos, L. Mao, C.D. Malliakas, M.G. Kanatzidis, Structure-bandgap relationships in hexagonal polytypes and low-dimensional structures of hybrid tin iodide perovskites. Inorg. Chem. 56(1), 56-73 (2017). https://doi.org/10.1021/ acs.inorgchem.6b02764

134. G. Kieslich, S. Sun, A.K. Cheetham, Solid-state principles applied to organic-inorganic perovskites: new tricks for an old dog. Chem. Sci. 5(12), 4712-4715 (2014). https://doi. org/10.1039/c4sc02211d

135. X. Jiang, F. Wang, Q. Wei, H. Li, Y. Shang et al., Ultra-high open-circuit voltage of tin perovskite solar cells via an electron transporting layer design. Nat. Commun. 11(1), 12451251 (2020). https://doi.org/10.1038/s41467-020-15078-2

136. C. Wang, F. Gu, Z. Zhao, H. Rao, Y. Qiu et al., Self-repairing tin-based perovskite solar cells with a breakthrough efficiency over 11. Adv. Mater. 32(31), e1907623-e1907631 (2020). https://doi.org/10.1002/adma.201907623

137. F. Hao, C.C. Stoumpos, R.P.H. Chang, M.G. Kanatzidis, Anomalous bandgap behavior in mixed $\mathrm{Sn}$ and $\mathrm{Pb}$ perovskites enables broadening of absorption spectrum in solar cells. J. Am. Chem. Soc. 136(22), 8094-8099 (2014). https://doi. org/10.1021/ja5033259

138. H.D. Kim, Y. Miyamoto, H. Kubota, T. Yamanari, H. Ohkita, Open-circuit voltage loss in $\mathrm{CH}_{3} \mathrm{NH}_{3} \mathrm{SnI}_{3}$ perovskite solar cells. Chem. Lett. 46, 253-256 (2017). https://doi. org/10.1246/cl.160994

139. T. Handa, T. Yamada, H. Kubota, S. Ise, Y. Miyamoto et al., Photocarrier recombination and injection dynamics in longterm stable lead-free $\mathrm{CH}_{3} \mathrm{NH}_{3} \mathrm{SnI}_{3}$ perovskite thin films and solar cells. J. Phys. Chem. C 121(30), 16158-16165 (2017). https://doi.org/10.1021/acs.jpcc.7b06199

140. W. Ke, P. Priyanka, S. Vegiraju, C.C. Stoumpos, I. Spanopoulos et al., Dopant-free tetrakis-triphenylamine hole transporting material for efficient tin-based perovskite solar cells. J. Am. Chem. Soc. 140(1), 388-393 (2018). https://doi. org/10.1021/jacs.7b10898

141. T. Yokoyama, T.-B. Song, D.H. Cao, C.C. Stoumpos, S. Aramaki et al., The origin of lower hole carrier concentration in methylammonium tin halide films grown by a vapor-assisted solution process. ACS Energy Lett. 2(1), 22-28 (2016). https ://doi.org/10.1021/acsenergylett.6b00513

142. S.A. Moyez, S. Roy, Thermal engineering of lead-free nanostructured $\mathrm{CH}_{3} \mathrm{NH}_{3} \mathrm{SnCl}_{3}$ perovskite material for thin-film solar cell. J. Nanopart. Res. 20(1), 5-17 (2017). https://doi. org/10.1007/s11051-017-4108-Z

143. N. Wang, Y. Zhou, M.-G. Ju, H.F. Garces, T. Ding et al., Heterojunction-depleted lead-free perovskite solar cells with coarse-grained b- $\gamma-\mathrm{CsSnI}_{3}$ thin films. Adv. Energy Mater. 6(24), 1601130-1601139 (2016). https://doi.org/10.1002/ aenm. 201601130

144. T.B. Song, T. Yokoyama, C.C. Stoumpos, J. Logsdon, D.H. Cao et al., Importance of reducing vapor atmosphere in the fabrication of tin-based perovskite solar cells. J. Am. Chem. Soc. 139(2), 836-842 (2017). https://doi.org/10.1021/ jacs.6b10734

145. B. Zhao, M. Abdi-Jalebi, M. Tabachnyk, H. Glass, V.S. Kamboj et al., High open-circuit voltages in tin-rich lowbandgap perovskite-based planar heterojunction photovoltaics. Adv. Mater. 29(2), 1604744-1604751 (2017). https://doi. org/10.1002/adma.201604744

146. S. Shao, J. Liu, G. Portale, H.-H. Fang, G.R. Blake et al., Highly reproducible Sn-based hybrid perovskite solar cells with 9\% efficiency. Adv. Energy Mater. 8(4), 17020191702028 (2018). https://doi.org/10.1002/aenm.201702019

147. L.-J. Chen, C.-R. Lee, Y.-J. Chuang, Z.-H. Wu, C. Chen, Synthesis and optical properties of lead-free cesium tin halide perovskite quantum rods with high-performance solar cell application. J. Phys. Chem. Lett. 7(24), 5028-5035 (2016). https://doi.org/10.1021/acs.jpclett.6b02344

148. J. Xi, Z. Wu, B. Jiao, H. Dong, C. Ran et al., Multichannel interdiffusion driven $\mathrm{FASnI}_{3}$ film formation using aqueous hybrid salt/polymer solutions toward flexible lead-free perovskite solar cells. Adv. Mater. 29(23), 1606964-1606970 (2017). https://doi.org/10.1002/adma.201606964

149. D. Moghe, L. Wang, C.J. Traverse, A. Redoute, M. Sponseller et al., All vapor-deposited lead-free doped $\mathrm{CsSnBr} 3$ planar solar cells. Nano Energy 28, 469-474 (2016). https:// doi.org/10.1016/j.nanoen.2016.09.009

150. C. Ran, J. Xi, W. Gao, F. Yuan, T. Lei et al., Bilateral interface engineering toward efficient 2D-3D bulk heterojunction tin halide lead-free perovskite solar cells. ACS Energy Lett. 3(3), 713-721 (2018). https://doi.org/10.1021/acsenergyl ett. 8 b00085

151. Y. Yu, D. Zhao, C.R. Grice, W. Meng, C. Wang et al., Thermally evaporated methylammonium tin triiodide thin films for lead-free perovskite solar cell fabrication. RSC Adv. 6(93), 90248-90254 (2016). https://doi.org/10.1039/c6ra1 $9476 \mathrm{a}$

152. C.-M. Tsai, N. Mohanta, C.-Y. Wang, Y.-P. Lin, Y.-W. Yang et al., Formation of stable tin perovskites co-crystallized with three halides for carbon-based mesoscopic lead-free perovskite solar cells. Angew. Chem. Int. Ed. 56(44), 13819-13823 (2017). https://doi.org/10.1002/anie.201707037

153. W. Li, J. Li, J. Li, J. Fan, Y. Maib et al., Addictive-assisted construction of all-inorganic CsSnIBr 2 mesoscopic perovskite solar cells with superior thermal stability up to 473 K. J. Mater. Chem. A 4, 17104-17110 (2016). https://doi. org/10.1039/c6ta08332c

154. K. Chen, P. Wu, W. Yang, R. Su, D. Luo et al., Lowdimensional perovskite interlayer for highly efficient leadfree formamidinium tin iodide perovskite solar cells. Nano Energy 49, 411-418 (2018). https://doi.org/10.1016/j.nanoe n.2018.05.006

155. E. Jokar, C.-H. Chien, A. Fathi, M. Rameez, C.-Y. Hao et al., Slow surface passivation and crystal relaxation with additives to improve device performance and durability for tinbased perovskite solar cells. Energy Environ. Sci. 11(9), 1-12 (2018). https://doi.org/10.1039/C8EE00956B 
156. X.Y. Meng, Y.B. Wang, J.B. Lin, X. Liu, X. He et al., Surface-controlled oriented growth of $\mathrm{FASnI}_{3}$ crystals for efficient lead-free perovskite solar cells. Joule 4, 902-912 (2020). https://doi.org/10.1016/j.joule.2020.03.007

157. L.-C. Tang, C.-S. Chang, J.Y. Huang, Electronic structure and optical properties of rhombohedral $\mathrm{CsGeI}_{3}$ crystal. J. Phys.: Condens. Matter 12(43), 9129-9143 (2000). https:// doi.org/10.1088/0953-8984/12/43/303

158. I. Kopacic, B. Friesenbichler, S.F. Hoefler, B. Kunert, H. Plank et al., Enhanced performance of germanium halide perovskite solar cells through compositional engineering. ACS Appl. Energy Mater. 1(2), 343-347 (2018). https://doi. org/10.1021/acsaem.8b00007

159. M.F. Mostafa, R.D. Willett, Magnetic properties of ferrous chloride complexes with two-dimensional structures. Phys. Rev. B 4(7), 2213-2215 (1971). https://doi.org/10.1103/ PhysRevB.4.2213

160. S.N. Ruddlesden, P. Popper, New compounds of the $\mathrm{K}_{2} \mathrm{NiF}_{4}$ type. Acta Cryst. 10(8), 538-539 (1957). https://doi. org/10.1107/S0365110X57001929

161. X.-P. Cui, K.-J. Jiang, J.-H. Huang, Q.-Q. Zhang, M.-J. $\mathrm{Su}$ et al., Cupric bromide hybrid perovskite heterojunction solar cells. Synth. Met. 209, 247-250 (2015). https://doi. org/10.1016/j.synthmet.2015.07.013

162. X. Li, B. Li, J. Chang, B. Ding, S. Zheng et al., (C6H5CH2NH3)2CuBr 4: a lead-free, highly stable twodimensional perovskite for solar cell applications. ACS Appl. Energy Mater. 1(6), 2709-2716 (2018). https://doi. org/10.1021/acsaem.8b00372

163. M. Abulikemu, S. Ould-Chikh, X. Miao, E. Alarousu, B. Murali et al., Optoelectronic and photovoltaic properties of the air-stable organohalide semiconductor $\left(\mathrm{CH}_{3} \mathrm{NH}_{3}\right)_{3} \mathrm{Bi}_{2} \mathrm{I}_{9}$. J. Mater. Chem. A 4(32), 12504-12515 (2016). https://doi. org/10.1039/c6ta04657f

164. R.L.Z. Hoye, R.E. Brandt, A. Osherov, V. Stevanović, S.D. Stranks et al., Methylammonium bismuth iodide as a leadfree, stable hybrid organic-inorganic solar absorber. Chem. Eur. J. 22(8), 2605-2610 (2016). https://doi.org/10.1002/ chem. 201505055

165. Z. Zhang, X. Li, X. Xia, Z. Wang, Z. Huang et al., High-quality $\left(\mathrm{CH}_{3} \mathrm{NH}_{3}\right)_{3} \mathrm{Bi}_{2} \mathrm{I}_{9}$ film-based solar cells: pushing efficiency up to 1.64. J. Phys. Chem. Lett. 8(17), 4300-4307 (2017). https://doi.org/10.1021/acs.jpclett.7b01952

166. C. Ran, Z. Wu, J. Xi, F. Yuan, H. Dong et al., Construction of compact methylammonium bismuth iodide film promoting lead-free inverted planar heterojunction organohalide solar cells with open-circuit voltage over 0.8 V. J. Phys. Chem. Lett. 8(2), 394-400 (2017). https://doi.org/10.1021/acs.jpcle tt.6b02578

167. M.B. Johansson, H. Zhu, E.M. Johansson, Extended photoconversion spectrum in low-toxic bismuth halide perovskite solar cells. J. Phys. Chem. Lett. 7(17), 3467-3471 (2016). https://doi.org/10.1021/acs.jpclett.6b01452

168. A.K. Baranwal, H. Masutani, H. Sugita, H. Kanda, S. Kanaya et al., Lead-free perovskite solar cells using $\mathrm{Sb}$ and Bi-based $\mathrm{A}_{3} \mathrm{~B}_{2} \mathrm{X}_{9}$ and $\mathrm{A}_{3} \mathrm{BX}_{6}$ crystals with normal and inverse cell structures. Nano Converg. 4(1), 26-39 (2017). https://doi. org/10.1186/s40580-017-0120-3

169. M. Lyu, J.-H. Yun, M. Cai, Y. Jiao, P.V. Bernhardt et al., Organic-inorganic bismuth (III)-based material: a lead-free, air-stable and solution-processable light-absorber beyond organolead perovskites. Nano Res. 9(3), 692-702 (2016). https://doi.org/10.1007/s12274-015-0948-y

170. S.S. Shin, O.P.C. Baena, O.C. Kurchin, O. Polizzotti, O.J. Yoo et al., Solvent-engineering method to deposit compact bismuth-based thin films: mechanism and application to photovoltaics. Chem. Mater. 30(2), 336-343 (2018). https://doi. org/10.1021/acs.chemmater.7b03227

171. S.M. Jain, D. Phuyal, M.L. Davies, M. Li, B. Philippe et al., An effective approach of vapour assisted morphological tailoring for reducing metal defect sites in leadfree, $\left(\mathrm{CH}_{3} \mathrm{NH}_{3}\right)_{3} \mathrm{Bi}_{2} \mathrm{I}_{9}$ bismuth-based perovskite solar cells for improved performance and long-term stability. Nano Energy 49, 614-624 (2018). https://doi.org/10.1016/j.nanoe n.2018.05.003

172. F. Bai, Y. Hu, Y. Hu, T. Qiu, X. Miao et al., Lead-free, airstable ultrathin $\mathrm{Cs}_{3} \mathrm{Bi}_{2} \mathrm{I}_{9}$ perovskite nanosheets for solar cells. Sol. Energy Mater. Sol. Cells 184, 15-21 (2018). https://doi. org/10.1016/j.solmat.2018.04.032

173. J.-C. Hebig, I. Kühn, J. Flohre, T. Kirchartz, Optoelectronic properties of $\left(\mathrm{CH}_{3} \mathrm{NH}_{3}\right)_{3} \mathrm{Sb}_{2} \mathrm{I}_{9}$ thin films for photovoltaic applications. ACS Energy Lett. 1(1), 309-314 (2016). https ://doi.org/10.1021/acsenergylett.6b00170

174. P.C. Harikesh, H.K. Mulmudi, B. Ghosh, T.W. Goh, Y.T. Teng et al., $\mathrm{Rb}$ as an alternative cation for templating inorganic lead-free perovskites for solution processed photovoltaics. Chem. Mater. 28(20), 7496-7504 (2016). https://doi. org/10.1021/acs.chemmater.6b03310

175. C. Zuo, L. Ding, Lead-free perovskite materials (NH4)3Sb2IxBr 9-x. Angew. Chem. Int. Ed. 56(23), 65286532 (2017). https://doi.org/10.1002/anie.201702265

176. F. Jiang, D. Yang, Y. Jiang, T. Liu, X. Zhao et al., Chlorineincorporation-induced formation of the layered phase for antimony-based lead-free perovskite solar cells. J. Am. Chem. Soc. 140(3), 1019-1027 (2018). https://doi.org/10.1021/ jacs.7b10739

177. W. Shockley, H.J. Queisser, Detailed balance limit of efficiency of p-n junction solar cells. J. Appl. Phys. 32(3), 510519 (1961). https://doi.org/10.1063/1.1736034

178. T. Li, X. Wang, Y. Yan, D.B. Mitzi, Phase stability and electronic structure of prospective sb-based mixed sulfide and iodide 3D perovskite $\left(\mathrm{CH}_{3} \mathrm{NH}_{3}\right) \mathrm{SbSI}_{2}$. J. Phys. Chem. Lett. 9(14), 3829-3833 (2018). https://doi.org/10.1021/acs.jpcle tt. 8 b01641

179. A. Antonio, Perovskite solar cells go lead free. Joule 1(4), 659-664 (2017). https://doi.org/10.1016/j.joule.2017.09.007

180. Y. Cai, W. Xie, H. Ding, Y. Chen, T. Krishnamoorthy et al., Computational study of halide perovskite-derived $\mathrm{A}_{2} \mathrm{BX}_{6}$ inorganic compounds: chemical trends in electronic structure and structural stability. Chem. Mater. 29(18), 7740 (2017). https://doi.org/10.1021/acs.chemmater.7b02013 
181. A.E. Maughan, J.A. Kurzman, J.R. Neilson, Hybrid inorganic-organic materials with an optoelectronically active aromatic cation: $\left(\mathrm{C}_{7} \mathrm{H}_{7}\right)_{2} \mathrm{SnI}_{6}$ and $\mathrm{C}_{7} \mathrm{H}_{7} \mathrm{PbI}_{3}$. Inorg. Chem. 54(1), 370-378 (2015). https://doi.org/10.1021/ic5025795

182. M.M. Elsenety, A. Kaltzoglou, M. Antoniadou, I. Koutselas, A.G. Kontos et al., Synthesis, characterization and use of highly stable trimethyl sulfonium tin(IV) halide defect perovskites in dye sensitized solar cells. Polyhedron 150, 83-91 (2018). https://doi.org/10.1016/j.poly.2018.05.001

183. X. Qiu, Y. Jiang, H. Zhang, Z. Qiu, S. Yuan et al., Lead-free mesoscopic $\mathrm{Cs}_{2} \mathrm{SnI}_{6}$ perovskite solar cells using different nanostructured $\mathrm{ZnO}$ nanorods as electron transport layers. Phys. Status Solid. RRL 10(8), 587-591 (2016). https://doi. org/10.1002/pssr.201600166

184. B. Lee, A. Krenselewski, S. Il-Baik, D. Seidman, R.P.H. Chang, Solution processing of air-stable molecular semiconducting iodosalts, $\mathrm{Cs}_{2} \mathrm{SnI}_{6-\mathrm{x}} \mathrm{Br}_{\mathrm{x}}$, for potential solar cell applications. Sustain. Energy Fuels 1(4), 710-724 (2017). https://doi.org/10.1039/c7se00100b

185. A.E. Maughan, A.M. Ganose, M.M. Bordelon, E.M. Miller, D.O. Scanlon et al., Defect tolerance to intolerance in the vacancy-ordered double perovskite semiconductors $\mathrm{Cs}_{2} \mathrm{SnI}_{6}$ and $\mathrm{Cs}_{2} \mathrm{TeI}_{6}$. J. Am. Chem. Soc. 138(27), 8453-8464 (2016). https://doi.org/10.1021/jacs.6b03207

186. M. Chen, M.-G. Ju, A.D. Carl, Y. Zong, R.L. Grimm et al., Cesium titanium(IV) bromide thin films based stable leadfree perovskite solar cells. Joule 2(3), 558-570 (2018). https ://doi.org/10.1016/j.joule.2018.01.009

187. E. Greul, M.L. Petrus, A. Binek, P. Docampo, T. Bein, Highly stable, phase pure $\mathrm{Cs} 2 \mathrm{AgBiBr} 6$ double perovskite thin films for optoelectronic applications. J. Mater. Chem. A 5(37), 19972-19981 (2017). https://doi.org/10.1039/c7ta06816f
188. C. Wu, Q. Zhang, Y. Liu, W. Luo, X. Guo et al., The dawn of lead-free perovskite solar cell: highly stable double perovskite Cs2AgBiBr 6 film. Adv. Sci. 5(3), 1700759-1700766 (2018). https://doi.org/10.1002/advs.201700759

189. W. Gao, C. Ran, J. Xi, B. Jiao, W. Zhang et al., High-quality $\mathrm{Cs} 2 \mathrm{AgBiBr} 6$ double perovskite film for lead-free inverted planar heterojunction solar cells with 2@2\% efficiency. ChemPhysChem 19(14), 1696-1700 (2018). https://doi. org/10.1002/cphc.201800346

190. M. Pantaler, K.T. Cho, V. Queloz, I.G. Benito, C. Fettkenhauer et al., Hysteresis-free lead-free double perovskite solar cells by interface engineering. ACS Energy Lett. 3, 17811786 (2018). https://doi.org/10.1021/acsenergylett.8b00871

191. M. Wang, P. Zeng, S. Bai, J. Gu, F. Li et al., High quality sequential-vapor-deposited $\mathrm{Cs} 2 \mathrm{AgBiBr} 6$ thin films for lead-free perovskite solar cells. Sol. RRL. 2(12), 18002171800222 (2018). https://doi.org/10.1002/solr.201800217

192. P. Li, W. Gao, C. Ran, H. Dong, X. Hou et al., Post-treatment engineering of vacuum-deposited $\mathrm{Cs}_{2} \mathrm{NaBiI}_{6}$ double perovskite film for enhanced photovoltaic performance. Phys. Status Solidi A 216(23), 1900567-1900574 (2019). https://doi. org/10.1002/pssa.201900567

193. H. Wang, Y. Zhao, Z. Wang, Y. Liu, Z. Zhao et al., Hermetic seal for perovskite solar cells: an improved plasma enhanced atomic layer deposition encapsulation. Nano Energy 69, 104375-104383 (2020). https://doi.org/10.1016/j.nanoe n.2019.104375

194. C. Chen, T.H. Han, S. Tan, J. Xue, Y. Zhao et al., Efficient flexible inorganic perovskite light-emitting diodes fabricated with $\mathrm{CsPbBr} 3$ emitters prepared via low-temperature in situ dynamic thermal crystallization. Nano Lett. 20(6), 46734680 (2020). https://doi.org/10.1021/acs.nanolett.0c01550 